\title{
Controlabilidade em Sistemas Hamiltonianos Afins
}

\section{Ivan Ruben Aguirre Cipe}

\author{
DISSERTAÇÃO APRESENTADA \\ $\mathrm{AO}$ \\ INSTITUTO DE MATEMÁTICA E ESTATÍSTICA \\ DA \\ UNIVERSIDADE DE SÃO PAULO \\ PARA OBTENÇÃO DO GRAU \\ $\mathrm{DE}$ \\ MESTRE EM MATEMÁTICA APLICADA
}

Área de Concentração: Matemática Aplicada

Orientador: Prof. Dr. Pedro Aladar Tonelli

Durante a elaboração deste trabalho, o autor recebeu apoio financeiro da CAPES

-São Paulo, novembro de 1996- 


\section{Controlabilidade em}

\section{Sistemas Hamiltonianos Afins}

Este exemplar corresponde à redlação final

da dissertação devidamente corrigida e

defendida por Ivan Ruben Aguirre Cipe

e aprovada pela comissão julgadora.

São Paulo, 13 de março 1997.

Banca examinadora:

- Prof. Dr. Pedro Aladar Tonelli (Orientador) - IME - USP

- Prof. Dr. Manuel Valentim de Pera Garcia - IME - USP

- Prof. Dr. Paulo Sérgio Pereira da Silva - EP -USP. 
doy gracias a Dios 


\title{
Resumo
}

Neste trabalho, é abordado o problema da controlabilidade em sistemas Hamiltonianos afins, sob aspecto de fortemente acessível e localmente fracamente observável, para o qual se define um isomorfismo entre a álgebra fortemente acessível e o espaço de observação, que nos dá uma relacão entre os critérios, e podemos concluir que um sistema Hamiltoniano afim de controle temos que fortemente acessível se e só se localmente é fracamente observável.

\begin{abstract}
In this work, we will study the problem of controllability in affine Hamiltonian system, under the aspect of strongly accessible and locally weakly observable, for which it is defined an isomorphism between the strong accessibility algebra and the observation space and this isomorphism gives us a connection between the criterious; and then we can conclude that an affine Hamiltonian control system is strongly accessible if and only if it is locally weakly observable.
\end{abstract}




\section{Agradecimentos}

\section{Gracias a mis Padres Berta e Ruben.}

Esțe trabalho é parte de um ideal que não teria sido possível realizá-lo sem a ajuda, o incentivo de meus Pais o companheirismo de amigos, colegas e professores. Estendo a todos a minha gratidão.

Sou grato em especial ao meu orientador e amigo Prof. Dr. Pedro Aladar Tonelli, pela sugestão do tema, pelos conselhos, pela nossa amizade, pela paciência ad infinitum durante o curso de Mestrado e orientação desta dissertação. Aos meus amigos que me apoiaram sempre Walo, Fily, Ruben e Ivett . Aos meus novos amigos que fiz por estas terras ao Flaco Jose Flores, Carolina, Gaspar e Carlos e a todas aquelas pessoas que deveriam estar aqui..

Pela oportunidade de poder melhorar a minha formação acadêmica e pelas facilidades dispensadas, agradeço à Universidade de São Paulo.

Agradeço a hospitalidade dos Brasileiros.

Agradeço aos meus Pais. 


\section{Sumário}

$\begin{array}{ll}\text { Introdução } & 1\end{array}$

1 Controlabilidade e Observabilidade 3

1.1 Introdução . . . . . . . . . . . . . . . . . . . 3

1.2 Sistema de Controle Afim Não-linear . . . . . . . . . . . . . . . 3

1.3 Acessibilidade . . . . . . . . . . . . . . . . . 5

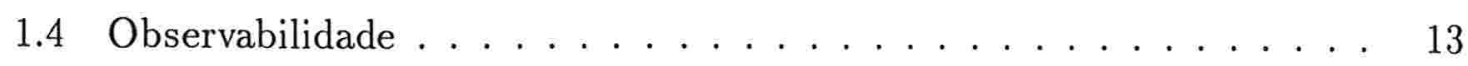

2 Geometria Simplética $\quad 33$

2.1 Introdução . . . . . . . . . . . . . . . . . . . . . 33

2.2 Variedade de Poisson . . . . . . . . . . . . . . . . . . . 33

2.3 Variedade Simplética . . . . . . . . . . . . . . . . . . . . . 39

3 Sistema Hamiltoniano de Controle $\quad 44$

3.1 Introdução . . . . . . . . . . . . . . . . . . . 44

3.2 Subvariedade Langrangiana . . . . . . . . . . . . . . . . . 45

3.3 Sistema Totalmente Hamiltoniano . . . . . . . . . . . . . . . . . 45

3.4 Sistema Hamiltoniano Afim . . . . . . . . . . . . . . . . . 46

3.5 Acessibilidade e Observabilidade . . . . . . . . . . . . . 51 


\section{Introdução}

Neste último século a formulação da mecânica clássica tem terminado numa elegante teoria descrita pelos textos [1] e [2], no entanto, a importância desta encontra-se na mecânica analítica, a mecânica analítica inspirou gerações de cientistas, que trouxeram grandes avanços em matemática . O trabalho em sistemas de controle Hamiltoniano foi iniciado por R. Brockett [3], como uma tentativa de apresentar a teoría de controle, desde o ponto de vista de um problema de mecânica analítica, envolvendo forças externas. Os exatos formalismos básicos e primeiros resultados são devido a van der Schaft. O presente trabalho foi inspirado na leitura dos artigos de van der Schaft [24] e [25]. Está dividido em três capítulos .

No capítulo 1, introduzimos os conceitos e teoremas principais de fortemente acessível e localmente fracamente observável em um sistema afim de controle não linear, damos algums exemplos para ilustrar os teoremas e conceitos de nosso estudo.

No capítulo 2 apresentamos alguns resultados de variedades de Poisson e variedades Simpléticas os quais utilizaremos para definir um sistema Hamiltoniano afim de controle.

No capítulo 3 apresentamos o que entenderemos por um sistema Hamiltoniano afim de controle e mostraremos que neste tipo de sistemas, temos a equivalência entre acessibilidade forte e observabilidade fraca, no sentido que fortemente acessível implica localmente fracamente observável e vice-versa o qual completa este presente trabalho. 


\section{Capítulo 1}

\section{Controlabilidade e Observabilidade}

\subsection{Introdução}

O objetivo deste capítulo é definir o que entenderemos por um sistema de controle afim não-linear fortemente acessível, fracamente observável e determinar alguns critérios para que se cumpra estas propriedades.

Os conceitos de controlabilidade e observabilidade em sistemas lineares foram introduzidos na teoria do controle por Kalman [10], [11] (na década de 60). Eles dão uma condição necessária e, em alguns casos, uma condição suficiente para que um problema dado possua solução. Também devemos mencionar a Kreindler e Sarachik [12] os quais desenvolveram independentemente os temas de controlabilidade e observabilidade. As questões similares para sistemas não-lineares não foram tratados efetivamente até 1970. A idéia de usar colchetes de Lie no estudo de acessibilidade pode ser creditada a Chow e também Hermann [8]. Podemos destacar os trabalhos de Brockett [4], Haynes-Hermes [6], trabalhando independentemente Lobry [13], Sussman-Jurdjevic [18] e Krener [13], desenvolveram algo análogo à controlabilidade linear para o caso não-linear em termos da álgebra de Lie de campo vetoriais. Não podemos deixar de mencionar os trabalhos da teoria do controle de Elliot [5], Hermann-Krener [7] e Sussman [19], [20], [22].

\subsection{Sistema de Controle Afim Não-linear}

Como proposto em [26] e argumentado em [24], [26], um sistema não-linear com entrada e saída pode ser descrito pelos seguintes elementos: 
i) $\mathcal{M}, \mathcal{W}$ variedades suaves.

ii) $\mathcal{B}$ é um fibrado vetorial sobre $\mathcal{M}$, com projeção $\pi: \mathcal{B} \longrightarrow \mathcal{M}$.

iii) Uma transformação suave $f: \mathcal{B} \longrightarrow T \mathcal{M} \times \mathcal{W}$

Tal que o diagrama abaixo comuta,

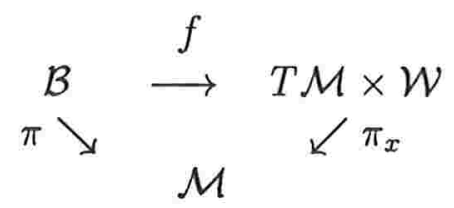

Onde $\mathcal{W}$ é o espaço de variáveis de saída, $\mathcal{M}$ o espaço de estados e a fibra $\pi^{-1}(x)$ em $\mathcal{B}, x \in \mathcal{M}$ representa o espaço de entrada e $\pi_{x}$ é a projeção natural de $T \mathcal{M}$ sobre $\mathcal{M}$, isto é, toma um vetor tangente $\alpha$ no ponto $x \in \mathcal{M}$ e associa $x$. Este conceito formaliza a idéia de que para cada $x \in \mathcal{M}$, temos um conjunto de possíveis velocidades ( elementos de $T \mathcal{M}$ ) e possíveis valores de variáveis de saída (elementos de $\mathcal{W})$ isto é, o espaço

$$
f\left(\pi^{-1}(x)\right) \subset T_{x} \mathcal{M} \times \mathcal{W}
$$

Em coordenadas locais $x$ para $\mathcal{M}, u$ para o fibrado de $\mathcal{B}, w$ para $\mathcal{W}$ e $f=(g, h)$ o sistema é dado por

$$
\begin{aligned}
& \dot{x}=g(x, u) \\
& w=h(x, u)
\end{aligned}
$$

Onde $g: \mathcal{B} \longrightarrow T \mathcal{M}$ e $h: \mathcal{B} \longrightarrow \mathcal{W}$ são transformações suaves. Denotaremos o sistema por $\Sigma(\mathcal{M}, \mathcal{W}, \mathcal{B}, f)$.

Um sistema afim é constituído por uma variedade suave $\mathcal{M}$, junto com uma distribuição diferenciável afim $\Delta$, ou seja, $\Delta(x)$ é um subespaço afim de $T_{x} \mathcal{M}$ para todo $x \in \mathcal{M}$, e uma transformação $h: \mathcal{M} \longrightarrow \mathcal{W}$ onde $\mathcal{W}$ é a variedade de saída. Em coordenadas locais um sistema de controle afim não-linear é representado por:

$$
\begin{aligned}
\dot{x} & =f(x)+\sum_{j=1}^{m} g_{j}(x) u_{j} \\
y_{j} & =h_{j}(x) \quad j=1, \ldots, p
\end{aligned}
$$

com $x=\left(x_{1}, \ldots, x_{n}\right)$ coordenadas para $\mathcal{M}$ (espaço estado) e $y=\left(y_{1}, \ldots, y_{p}\right)$ coordenadas para $\mathcal{W}, u=\left(u_{1}, \ldots, u_{m}\right) \in \mathcal{U}, \mathcal{U} \subset \mathbb{R}^{m}$, compacto é o espaço de controle e $f, g_{1}, \ldots, g_{m}$ são campos vetoriais suaves definidos em $\mathcal{M}$, tal que $\Delta(x)=$ $f(x)+\operatorname{Span}\left\{g_{1}(x), \ldots, g_{m}(x)\right\}$. saída.

A transformação $h:\left(h_{1}, \ldots, h_{p}\right)^{t}: \mathcal{M} \longrightarrow \mathcal{W} \subset \mathbb{R}^{p}$ é chamada transformação de 
Assumiremos que o sistema (1) é completo, i.e, para todo controle limitado e mensurável $u(t)$ e todo $x_{0} \in \mathcal{M}$ existe uma solução da equação (1) satisfazendo a condição $x\left(t_{0}\right)=x_{0}$ e $x(t) \in \mathcal{M}, \forall t \in \mathbb{R}$, esta condição não é muito restritiva, dado que para sistemas tempo-contínuo, completo é equivalente a $\mathrm{V}$-completo, onde $V \subset \mathcal{U}$ e $\mathcal{U}$ compacto para maiores detalhes veja [17], pagina 96 .

\subsection{Acessibilidade}

Sejam $\mathcal{V}$ uma vizinhança de $x_{0}$ e $T>0 ; \mathcal{R}^{\mathcal{V}}\left(x_{0}, T\right)$ denota o conjunto dos pontos atingíveis desde $x_{0}$ num tempo $T>0$, seguindo uma trajetória à qual permanece para $t \leq T$ na vizinhança $\mathcal{V}$ de $x_{0}$, isto é,

$$
\begin{aligned}
\mathcal{R}^{\mathcal{V}}\left(x_{0}, T\right)= & \{x \in \mathcal{M}: \text { existe um controle } u:[0, T] \rightarrow \mathcal{U} \\
& \left.\operatorname{com} x(0)=x_{0}, x(t) \in \mathcal{V}, 0 \leq t \leq T, x(T)=x\right\} .
\end{aligned}
$$

Denotaremos por:

$$
\mathcal{R}_{T}^{\mathcal{V}}\left(x_{0}\right)=\bigcup_{\tau \leq T} \mathcal{R}^{\mathcal{V}}\left(x_{0}, \tau\right)
$$

Encontrar os pontos atingíveis desde $x_{0} \in \mathcal{M}$ em um tempo finito é o que origina o problema de acessibilidade.

Definição 1.3.1 Seja $\mathcal{V}$ um subconjunto de $\mathcal{M}$,diremos que $x_{1}$ é $\mathcal{V}$-acessível desde $x_{0}$ e denotaremos por $x_{1} \mathcal{A}_{\mathcal{V}} x_{0}$, se existe um controle limitado mensurável $u(t):\left[t_{0}, t_{1}\right] \rightarrow$ $\mathcal{U} \subset \mathbb{R}^{m}$, tal que a correspondente solução $x(t):\left[t_{0}, t_{1}\right] \rightarrow \mathcal{M}$ da equação diferencial (1) satisfaz

$$
x\left(t_{0}\right)=x_{0}, \quad x\left(t_{1}\right)=x_{1} \quad \text { e } x(t) \in \mathcal{V}
$$

para todo $t \in\left[t_{0}, t_{1}\right]$.

Observação 1.3.1 Em geral, $\mathcal{M}$-acessivel e $\mathcal{A}_{\mathcal{M}}$ são referidos simplesmente como acessibilidade e $\mathcal{A}$. $\mathcal{A}\left(x_{0}\right)$ denotará o conjunto de pontos acessiveis desde $x_{0}$.

Dado $x_{0} \in \mathcal{M}$, o que podemos dizer do conjunto dos pontos que podem ser atingidos desde $x_{0}$ em tempo finito para a escolha de um controle $u$ é o problema de controlabilidade.

Definição 1.3.2 O Sistema (1) é dito controlável em $x_{0}$ se $\mathcal{A}\left(x_{0}\right)=\mathcal{M}$ e controlável se $\mathcal{A}(x)=\mathcal{M}$ para todo $x \in \mathcal{M}$. 
Un espaço vetorial $V$ (sobre $\mathbb{R}$ ) é um algebra de Lie, se existe uma operação binaria $V \times V \longrightarrow V$, denotada $[\cdot, \cdot]$ e satisfaz:

i) Bilinearidad:

$$
\left[\alpha_{1} v_{1}+\alpha_{2} v_{2}, v_{3}\right]=\alpha_{1}\left[v_{1}, v_{3}\right]+\alpha_{2}\left[v_{2}, v_{3}\right]
$$

ii) Antisimétrica:

$$
\left[v_{1}, v_{2}\right]=-\left[v_{2}, v_{1}\right]
$$

iii) Identidade de Jacobi:

$$
\left[v_{1},\left[v_{2}, v_{3}\right]\right]+\left[v_{2},\left[v_{3}, v_{1}\right]\right]+\left[v_{3},\left[v_{1}, v_{2}\right]\right]=0 \quad \forall v_{1}, v_{2}, v_{3} \in V
$$

Uma subálgebra de uma álgebra de Lie $(V,[\cdot, \cdot])$ é um subespaço vetorial $V_{1} \subset V$ tal que $\left[v_{1}, w_{1}\right] \in V_{1} \quad \forall v_{1}, w_{1} \in V_{1}$.

Denotaremos por $V^{\infty}(\mathcal{M})$ o conjunto dos campos vetoriais suaves em $\mathcal{M}$, que possui uma estrutura de álgebra de Lie mediante a seguinte operação binária $[\cdot, \cdot]$ : $V^{\infty}(\mathcal{M}) \times V^{\infty}(\mathcal{M}) \longrightarrow V^{\infty}(\mathcal{M})$, chamamos $[\cdot, \cdot]$ o colchete de Lie de campos vetoriais.

Definição 1.3.3 : Consideremos o sistema (1). A álgebra acessivel $\mathcal{C}$ é a menor subálgebra de $V^{\infty}(M)$ que contém $f, g_{1}, \ldots, g_{m}$. A distribuição acessível C. é a distribuiçâo gerada pela álgebra acessivel $\mathcal{C}$.

Denotaremos por

$$
C(x)=\operatorname{Span}\{X(x) / X \text { campo vetorial em } \mathcal{C}\}, \quad x \in \mathcal{M} .
$$

Vejamos uma caracterização de $\mathcal{C}$ mediante a seguinte proposição.

Proposição 1.3.1 Todo elemento de $\mathcal{C}$ é uma combinação linear sobre $\mathbb{R}$ de repetidos colchetes de Lie da forma

$$
\left[X_{k},\left[X_{k-1} \cdot\left[\ldots\left[X_{1}, X_{0}\right] \ldots\right]\right]\right]
$$

onde $X_{i}$, pertence ao conjunto $\left\{f, g_{1}, \ldots, g_{m}\right\}$, para $0 \leq i \leq k, k \in I N$.

Demonstração: Seja $\mathcal{L}$ o subespaço vetorial de $V^{r \infty}(M)$ gerado por

$$
\left[X_{k},\left[X_{k-1},\left[\ldots\left[X_{1}, X_{0}\right] \ldots\right]\right]\right]
$$


o comprimento desta expressão é o numero de colchetes de Lie i.e k. Temos que $\mathcal{L} \subset \mathcal{C}$ (pela definição de $\mathcal{C}$ ). Mostraremos que $\mathcal{L}=\mathcal{C}$. Para isso basta provar que $\mathcal{L}$ é uma subálgebra. De fato, tomemos dois elementos arbitrários de $(*)$

$$
\begin{aligned}
\mathcal{Z} & =\left[Z_{j},\left[Z_{j-1},\left[\ldots\left[Z_{1}, Z_{0}\right] \ldots\right]\right]\right] \\
\mathcal{Y} & =\left[Y_{l},\left[Y_{l-1},\left[\ldots\left[Y_{1}, Y_{0}\right] \ldots\right]\right]\right]
\end{aligned}
$$

com comprimentos $j$ e $l$ respectivamente. Por indução provemos que $[\mathcal{Z}, \mathcal{Y}] \in \mathcal{L}$, para qualquer $j$ e $l$. Fixemos $l$ arbitrário e apliquemos indução sobre j. Para $j \leq 1$ isto é verdade. Por hipótese de indução, temos que

$$
[\mathcal{Z}, \mathcal{Y}] \in \mathcal{L} \text { para } j \leq k .
$$

Agora tomemos $j=k+1$, se tomamos

$$
\mathcal{Z}^{1}=\left[Z_{j-1},\left[\ldots\left[Z_{1}, Z_{0}\right] \ldots\right]\right]
$$

pela identidade de Jacobi

$$
\left[\left[Z_{j}, \mathcal{Z}^{1}\right], \mathcal{Y}\right]+\left[\left[\mathcal{Z}^{1}, \mathcal{Y}\right], Z_{j}\right]+\left[\left[\mathcal{Y}, Z_{j}\right], \mathcal{Z}^{1}\right]=0
$$

e como $\left[\left[Z_{j}, \mathcal{Z}^{1}\right], Y\right]=[\mathcal{Z}, \mathcal{Y}]$, temos

$$
\begin{aligned}
{[\mathcal{Z}, \mathcal{Y}] } & =-\left[\left[\mathcal{Z}^{1}, \mathcal{Y}\right], Z_{j}\right]-\left[\left[\mathcal{Y}, Z_{j}\right], \mathcal{Z}^{1}\right] \\
& =\left[Z_{j},\left[\mathcal{Z}^{1}, \mathcal{Y}\right]\right]+\left[\mathcal{Z}^{1},\left[\mathcal{Y}, Z_{j}\right]\right] \\
& =\left[Z_{j},\left[\mathcal{Z}^{1}, \mathcal{Y}\right]\right]-\left[\mathcal{Z}^{1},\left[Z_{j}, \mathcal{Y}\right]\right]
\end{aligned}
$$

$\mathcal{Z}^{1}$ tem comprimento $j-1=k$, segue da hipótese de indução que $\left[\mathcal{Z}^{1}, \mathcal{Y}\right] \in \mathcal{L}$ e, portanto, o primeiro termo da direita pertence a $\mathcal{L}$, assim também o segundo termo da direita pertence a $\mathcal{L}$. Logo $[\mathcal{Z}, \mathcal{Y}] \in \mathcal{L}$ e, portanto, $\mathcal{L}$ é uma subálgebra que contém $\left\{f, g_{1}, \ldots, g_{m}\right\}$, logo pela definição

$$
\mathcal{L}=\mathcal{C} .
$$

Vejamos agora um Teorema que nos será de grande utilidade para justificar algumas propiedades.

Teorema 1.3.1 Seja o sistema (1). Se $\operatorname{dim} C\left(x_{0}\right)=n$ então para qualquer vizinhança $\mathcal{V}$ de $x_{0}$ e $T>0$, o conjunto $\mathcal{R}_{T}^{\mathcal{V}}\left(x_{0}\right)$ contém um conjunto aberto não-vazio de $\mathcal{M}$. 
Demonstração: Decorre da hipótese que existe uma vizinhança $V \subset \mathcal{V}$ de $x_{0}$ tal que $\operatorname{dim} C(x)=n \forall x \in V$. Construíremos uma seqüência de subvariedades $\mathcal{N}_{k}$ em $V \operatorname{com} \operatorname{dim} \mathcal{N}_{k}=k, 1 \leq k \leq n$. Seja $\mathcal{F}$ o conjunto de campos vetoriais associados ao sistema (1), isto é $\mathcal{F}=\left\{f+\sum_{j=1}^{m} g_{j} u_{j}:\left(u_{1}, \ldots, u_{m}\right) \in \mathcal{U}\right\}$.

Para $k=1$ escolhamos $X_{1} \in \mathcal{F}$ tal que $X_{1}\left(x_{0}\right) \neq 0$ então pelo Teorema do Fluxo Tubular para $\epsilon>0$ suficientemente pequeno temos:

$$
\mathcal{N}_{1}=\left\{X_{1}^{t_{1}}\left(x_{0}\right) \backslash 0<t_{1}<\epsilon_{1}\right\}
$$

é uma subvariedade mergulhada de $\mathcal{M}$ com $\operatorname{dim} \mathcal{N}_{1}=1$ e $\mathcal{N}_{1} \subset V$. Agora construiremos $\mathcal{N}_{k}$ por inducão para $k>1$. Suponhamos agora que $\mathcal{N}_{k-1} \subset V$ é uma subvariedade tal que $\operatorname{dim} \mathcal{N}_{k-1}=k-1$, e definida por:

$$
\mathcal{N}_{k-1}=\left\{X_{k-1}^{t_{k-1}} \circ X_{k-2}^{t_{k-2}} \circ \ldots \circ X_{1}^{t_{1}}\left(x_{0}\right) \backslash 0 \leq \sigma_{i}<t_{i}<\epsilon_{i}, 1 \leq i \leq k-1\right\}
$$

onde os $X_{i}, 1 \leq i \leq k-1$, são campos vetoriais em $\mathcal{F}$ e $\sum_{i=1}^{k-1} \sigma_{i}$ é suficientemente pequeno. Se $k-1<n$, suponhamos que $\left.X_{(} q\right) \in T_{q} \mathcal{N}_{k-1}$ para qualquer $X \in \mathcal{F}$ e $q \in \mathcal{N}_{k-1}$, isto implica que $\operatorname{dim} C(q)<n, \forall q \in N_{k-1} \subset V$, o que contraria a definição de $V$, logo podemos encontrar $X_{k} \in \mathcal{F}$ e $q \in \mathcal{N}_{k-1}$ tal que

$$
X_{k}(q) \notin T_{q} \mathcal{N}_{k-1}
$$

Portanto, podemos tomar $q$ arbitrariamente próximo de $x_{0}$ tal que a transformação

$$
\left(t_{k}, \ldots, t_{1}\right) \longmapsto X_{k}^{t_{k}} \circ X_{k-1}^{t_{k-1}} \circ \ldots \circ X_{1}^{t_{1}}\left(x_{0}\right)
$$

tem posto igual a $k$ no conjunto $0 \leq \sigma_{i}<t_{i}<\epsilon_{i}, 1 \leq i \leq k$, pelo Teorema do Posto, a imagem da transformação para $\epsilon_{i}, 1 \leq i \leq k$ suficientemente pequeno é uma subvariedade mergulhada $\mathcal{N}_{k} \subset V$ de dimensão $k$, e podemos continuar com este procedimento ate que $k=n$ e podemos concluir que $\mathcal{N}_{n}$ é um conjunto aberto contido em $\mathcal{R}_{T}^{\mathcal{V}}\left(x_{0}\right)$.

Definição 1.3.4 Diremos que o sistema (1) é localmente acessivel desde $x_{0}$ quando $\mathcal{R}_{T}^{\mathcal{V}}\left(x_{0}\right)$ contém um conjunto aberto não-vazio de $\mathcal{M}$ para toda vizinhança $\mathcal{V}$ de $x_{0} e$ todo $T>0$. Se isto ocorre para todo $x_{0} \in \mathcal{M}$, então o sistema é chamado localmente acessivel.

Corolário 1.3.1 Se $\operatorname{dim} C(x)=n$ para todo $x \in \mathcal{M}$, entâo o sistema (1) e localmente acessivel. 
Demonstração: É uma conseqüência do Teorema.

A proposição a seguir nos permite encontrar uma subvariedade de $\mathcal{M}$ onde o sistema (1) restrito a essa subvariedade é localmente acessível.

Proposição 1.3.2 Se dim $C=k$ numa vizinhança de $x_{0}, k<n$. Então podemos encontrar vizinhanças $\mathcal{W}$ de $x_{0}$ e coordenadas locais $x_{1}, \ldots, x_{n}$ tal que a subvariedade

$$
\mathcal{S}_{x_{o}}=\left\{q \in \mathcal{W} \backslash x_{i}(q)=x_{i}\left(x_{0}\right) i=k+1, \ldots, n\right\}
$$

é uma variedade integral de $C$. Então para qualquer vizinhança $\mathcal{V} \subset \mathcal{W}$ de $x_{0}$ e para todo $T>0, \mathcal{R}_{T}^{\mathcal{V}}\left(x_{0}\right)$ está contida em $\mathcal{S}_{x_{0}}$. Além disso, $\mathcal{R}_{T}^{\mathcal{V}}\left(x_{0}\right)$ contém um conjunto aberto não-vazio da variedade integral $\mathcal{S}_{x_{0}}$ o sistema (1) restrito a esta variedade é localmente acessivel.

Demonstração: Decorre do Teorema de Frobenius a primeira parte, pois $C$ é involutiva. Temos que $f(x)+\sum_{j=1}^{m} g_{j}(x) u_{j} \in C(x)$ para todo $u=\left(u_{1}, \ldots, u_{m}\right) \in \mathcal{U}$ e $x \in \mathcal{M}$. O sistema (1) para $x(0)=x_{0}$ pode ser restrito a $\mathcal{S}_{x_{0}}$ (para $t$ suficientemente pequeno), e temos que $\operatorname{dim} \mathcal{S}_{x_{0}}=\operatorname{dim} C\left(x_{0}\right)$ onde podemos aplicar o Corolário (1.3.1) a este sistema restrito.

Mesmo que o sistema seja controlável em $x_{0}$ pode ser necesário percorrer uma considerável distância ou um tempo grande para alcançar pontos próximos de $x_{0}$, por isso introduziremos o conceito de controlabilidade local.

Definição 1.3.5 O sistema (1) é controlável localmente em $x_{0}$ se para toda vizinhança $\mathcal{V}$ de $x_{0}, A_{\mathcal{V}}\left(x_{0}\right)$ é também uma vizinhança de $x_{0}$. O sistema (1) é controlável localmente se é localmente controlável para todo $x \in \mathcal{M}$.

A relação de acessibilidade é reflexiva e transitiva para sistemas não-lineares (não é simétrica), é possível definir uma relação fraca que seja de equivalência isto dá origem aos conceitos de $\mathcal{V}$-acessibilidade fraca, fracamente controlável, para maiores detalhes veja [7].

Exemplo 1.3.1 Consideremos o seguinte sistema:

$$
\begin{aligned}
& \dot{x}=u \\
& \dot{y}=x^{2}
\end{aligned}
$$

Para qualquer ponto $(x, y)$ no plano, só a região sobre o plano y é acessível, basta tomar $x_{1}$ e $x_{2}$ tal que $x_{1}<x_{2}$, para ver que a acessibilidade não é simétrica (figura 1.1). 


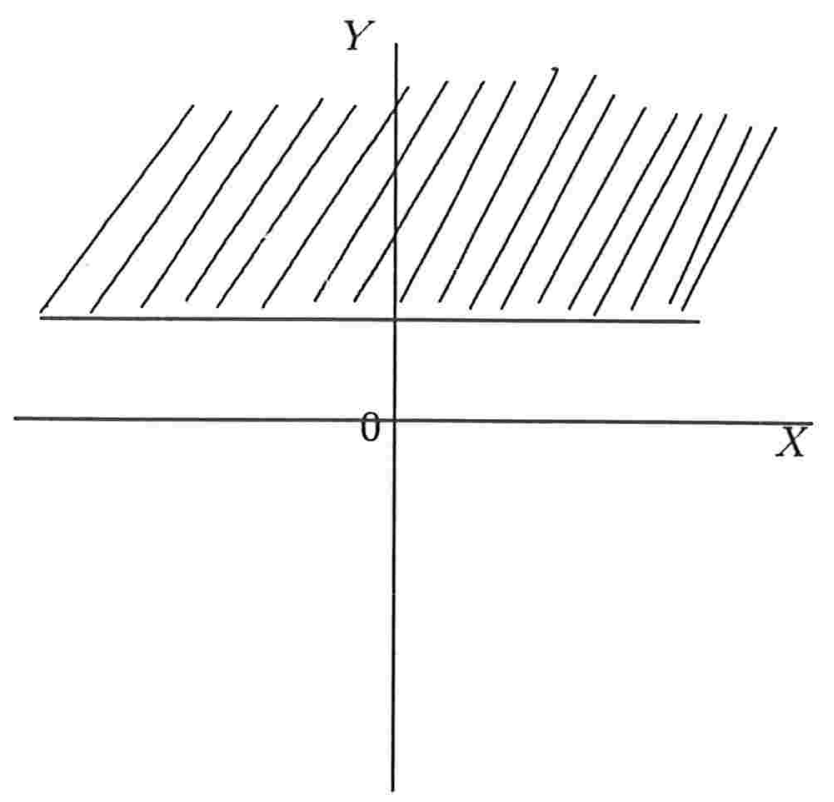

Figura 1.1:

Definição 1.3.6 Dizemos que o sistema (1) é fortemente acessivel desde $x_{0}$ se $\mathcal{R}^{\mathcal{M}}\left(x_{0}, T\right)$ tem interior não-vazio para algum T. Se isto ocorre para todo $x_{0} \in \mathcal{M}$ temos que o sistema (1) é chamado fortemente acessivel.

Em [16] fortemente acessível é definido como localmente acessível, a definição a seguir nos levará a um critério para saber quando um sistema é fortemente acessível.

Definição 1.3.7 Consideremos o sistema (1). A álgebra fortemente acessivel $\mathcal{C}_{0}$ é a menor sub-álgebra a qual contém os campos $g_{1}, \ldots, g_{m}$ e satisfaz $[f, X] \in \mathcal{C}_{0}$ para todo $X \in \mathcal{C}_{0}$. Uma distribuição fortemente acessivel $C_{0}$ é a distribução involutiva gerada por $\mathcal{C}_{0}$, onde

$$
C_{0}(x)=\operatorname{Span}\left\{X(x) \backslash X \text { campo vetorial em } \mathcal{C}_{0}\right\}
$$

Se no sistema (1), temos que $f$ é uma combinação linear de $\left\{g_{1}, \ldots, g_{m}\right\}$ então $\mathcal{C}=\mathcal{C}_{0}$, significa fisicamente que of $f$ pode ser anulado por uma entrada adequada e o sistema equivale a um sistema sim drift. Temos que $C_{0}+\operatorname{span}\{f\} \subset C$ e se $x$ é um ponto regular de $C_{0}+\operatorname{span}\{f\}$ então $\left(C_{0}+\operatorname{span}\{f\}\right)(x)=C(x)$, veja maiores detalhes em [9] pagina 61 .

Vejamos uma caracterização de $\mathcal{C}_{0}$, mediante a seguinte proposição. 
Proposição 1.3.3 Todo elemento de $\mathcal{C}_{0}$ é uma combinação linear de repetidos colchetes de Lie da forma

$$
\left[X_{k},\left[X_{k-1},\left[\ldots\left[X_{1}, g_{j}\right] \ldots\right]\right]\right] \quad 1 \leq j \leq m \quad k=1, \ldots
$$

onde cada $X_{i}$ pertence ao conjunto $\left\{f, g_{1}, \ldots, g_{m}\right\}, 1 \leq i \leq k$.

Demonstração: Seja $\mathcal{L}_{0}$ o subespaço linear de $V^{\infty}(\mathcal{M})$ gerado pelos campos $(*)$, o comprimento desta expressão é o número de colchetes de Lie, i.e k. Decorre da definição de $\mathcal{C}_{0}$ que $\mathcal{L}_{0} \subset \mathcal{C}_{0}$. Mostremos que $\mathcal{L}_{0}=\mathcal{C}_{0}$. Para isso basta provar que $\mathcal{L}_{0}$ é um subálgebra. Seja

$$
\begin{aligned}
\mathcal{Z} & =\left[Z_{p},\left[Z_{p-1},\left[\ldots\left[Z_{1}, g_{i}\right] \ldots\right]\right]\right] \\
\mathcal{Y} & =\left[Y_{l},\left[Y_{l-1},\left[\ldots\left[Y_{1}, g_{n}\right] \ldots\right]\right]\right]
\end{aligned}
$$

elementos pertencentes a $(*)$, de comprimento $p$ e $l$ respectivamente. Por indução provemos que $[\mathcal{Z}, \mathcal{Y}] \in \mathcal{L}_{0}$, para qualquer $p$ e $l$. Assumamos que $[\mathcal{Z}, \mathcal{Y}] \in \mathcal{L}_{0}$, para todo $\mathcal{Y}, l$ arbitrário e para todo $p \leq k$. Isto é verdade para $k=1$. Pela hipótese de indução assumamos que é valido $p \leq k$. Provemos que é válido para $p=k+1$. Tomando-se $\mathcal{Z}^{1}=\left[Z_{p-1},\left[Z_{p-2},\left[\ldots\left[Z_{1}, g_{i}\right] \ldots\right]\right]\right]$ pela identidade de Jacobi temos:

$$
\left[\left[Z_{p}, \mathcal{Z}^{1}\right], \mathcal{Y}\right]+\left[\left[\mathcal{Z}^{1}, \mathcal{Y}\right], Z_{p}\right]+\left[\left[\mathcal{Y}, Z_{p}\right], \mathcal{Z}^{1}\right]=0
$$

mas, $\left[\left[Z_{p}, \mathcal{Z}^{1}\right], \mathcal{Y}\right]=[\mathcal{Z}, \mathcal{Y}]$ logo,

$$
\begin{aligned}
{[\mathcal{Z}, \mathcal{Y}] } & =-\left[\left[\mathcal{Z}^{1}, \mathcal{Y}\right], Z_{p}\right]-\left[\left[\mathcal{Y}, Z_{p}\right], \mathcal{Z}^{1}\right] \\
& =\left[Z_{p},\left[\mathcal{Z}^{1}, \mathcal{Y}\right]\right]-\left[\mathcal{Z}^{1},\left[Z_{p}, \mathcal{Y}\right]\right]
\end{aligned}
$$

$\mathcal{Z}^{1}$ tem comprimento $j-1=k$, segue da hipótese de indução que $\left[\mathcal{Z}^{1}, \mathcal{Y}\right] \in \mathcal{L}_{0}$ e portanto, o primeiro termo da direita pertence a $\mathcal{L}_{0}$ e, assim também o segundo termo da direita pertence a $\mathcal{L}_{0}$. Logo

$$
[\mathcal{Z}, \mathcal{Y}] \in \mathcal{L}_{0}
$$

portanto $\mathcal{L}_{0}$ é uma subálgebra e por definição

$$
\mathcal{L}_{0}=\mathcal{C}_{0} .
$$

O Teorema a seguir nos dá um critério para que um sistema seja fortemente acessível.

Teorema 1.3.2 Se $C_{0}(x)=T_{x} \mathcal{M}$ para todo $x \in \mathcal{M}$, então o sistema (1) é fortemente acessivel. 
Demonstração: Por hipótese

$$
C_{0}\left(x_{0}\right)=T_{x_{0}} \mathcal{M}
$$

$\log 0$

$$
\operatorname{dim} C_{0}\left(x_{0}\right)=\operatorname{dim} \mathcal{M}=n
$$

reduziremos a nossa demonstração para poder aplicar o Teorema (1.3.1). Para isso acrescentaremos ao espaço de estado da equação (1) a equação $\dot{t}=1$. Obtendo assim o sistema

$$
\begin{aligned}
& \dot{x}=f(x)+\sum_{j=1}^{m} g_{j}(x) u_{j} \\
& \dot{t}=1
\end{aligned}
$$

onde $\tilde{\mathcal{M}}=\mathcal{M} \times \mathbb{R}$, espaço de estado $\tilde{x}=(x, t) \mathrm{e}$

$$
\tilde{f}(x, t)=f(x) \frac{\partial}{\partial x}+\frac{\partial}{\partial t}, \quad \tilde{g}_{j}(x, t)=g_{j}(x) \frac{\partial}{\partial x} \quad j=1, \ldots, m
$$

são campos vetoriais definidos en $\tilde{\mathcal{M}}$, podemos concluir que a distribuição acessível $\tilde{C}$ do sistema aumentado satisfaz

Portanto

$$
\begin{gathered}
\tilde{C}_{0}\left(x_{0}, t_{0}\right)=C_{0}\left(x_{0}\right) \\
\tilde{C}\left(x_{0}, t_{0}\right)=\tilde{C}_{0}\left(x_{0}, t_{0}\right)+\operatorname{Span}\left\{f\left(x_{0}\right)+\frac{\partial}{\partial t}\right\}
\end{gathered}
$$

$$
\operatorname{dim} \tilde{C}\left(x_{0}, t_{0}\right)=\operatorname{dim} C_{0}\left(x_{0}\right)+1 \quad \forall t_{0} \in \mathbb{R}
$$

em particular, temos

$$
\operatorname{dim} \tilde{C}\left(x_{0}, 0\right)=n+1
$$

Pelo Teorema (1.3.1), podemos concluir que, para qualquer $T>0$ e $\mathcal{V}$ uma vizinhança de $x_{0}$, tal que $\tilde{\mathcal{V}}=\mathcal{V} \times(-\epsilon, T+\epsilon), \epsilon>0$, o conjunto $\mathcal{R}_{T}^{\tilde{\mathcal{V}}}\left(x_{0}, 0\right)$ contém um conjunto aberto não-vazio de $\tilde{\mathcal{M}}$, onde $\tilde{\mathcal{V}} \subset \tilde{\mathcal{M}}$ é uma vizinhança de $\left(x_{0}, 0\right)$. Portanto existe $\mathcal{W} \subset \mathcal{M}$ aberto não-vazio e um intervalo $(a, b)$, com $0<a<b \leq T$ tal que $\mathcal{W} \times(a, b) \subset$ $\mathcal{R}_{T}^{\tilde{\mathcal{V}}}\left(\left(x_{0}, 0\right)\right)$.

Por outro lado, seja $\mathcal{T} \in(a, b), \mathcal{W} \times\{\mathcal{T}\} \subset \mathcal{R}_{T}^{\overline{\mathcal{V}}}\left(\left(x_{0}, 0\right)\right)$, e podemos dizer que $\mathcal{W} \subset \mathcal{R}^{\mathcal{V}}\left(x_{0}, \mathcal{T}\right)$. Portanto, o interior de $\mathcal{R}^{\mathcal{M}}\left(x_{0}, T\right)$ é não-vazio para algum $T$ (pode ser $T=\mathcal{T})$.

Isso demonstra o teorema. 
Observação 1.3.2 Quando o sistema (1) é analítico (os campos são analíticos), temos que fortemente acessivel e a condição $C(x)=T_{x} \mathcal{M}$, para todo $x \in \mathcal{M}$ são equivalentes, para maiores detalhes veja [18].

Vejamos uma definição local para Fortemente Acessível.

Definição 1.3.8 O sistema (1) é localmente fortemente acessivel desde $x_{0}$, se para toda vizinhança $\mathcal{V}$ de $x_{0}$, o conjunto $\mathcal{R}^{\mathcal{V}}\left(x_{0}, T\right)$ contém um subconjunto aberto nãovazio para qualquer $T>0$ suficientemente pequeno.

Como conseqüência do Teorema (1.3.2) temos

Teorema 1.3.3 Seja o sistema (1). Se $C_{0}\left(x_{0}\right)=T_{x_{0}} \mathcal{M}$, então o sistema é localmente fortemente acessivel desde $x_{0}$.

Demonstração: Retomemos a prova do Teorema (1.3.2), definamos a transformação $\Lambda: \mathcal{W} \longrightarrow \tilde{\mathcal{W}}$, onde $\tilde{\mathcal{W}} \subset \mathcal{R}^{U}\left(x_{0}, T\right)$ é aberto e $U$ vizinhança de $x_{0}$, tal que $\Lambda(x)=$ $X^{T-\mathcal{T}}(x), X \in \mathcal{F}$. Escolhendo $T$ suficientemente pequeno, $\tilde{W} \cap \mathcal{R}^{\mathcal{V}}\left(x_{0}, T\right)$ contém um conjunto aberto não-vazio de $\mathcal{M}$. Portanto o sistema (1) é localmente fortemente acessível.

\subsection{Observabilidade}

Observação 1.4.1 Denotaremos por $y\left(t, 0, x_{0}, u\right)=h\left(x\left(t, 0, x_{0}, u\right)\right)$ a saída do sistema (1) para um controle $u$, com estado inicial $x(0)=x_{0}$.

Definição 1.4.1 Dizemos que dois estados $x_{1}, x_{2} \in \mathcal{M}$ são indistinguíveis (denotado $x_{1} \mathcal{I} x_{2}$ ) para o sistema (1), se para todo controle admissivel u a função de saída $t \longrightarrow y\left(t, 0, x_{1}, u\right), t \geq 0$ do sistema para um estado inicial $x(0)=x_{1}$ e a função de saida $t \longrightarrow y\left(t, 0, x_{2}, u\right), t \geq 0$ do sistema para um estado inicial $x(0)=x_{2}$ são idênticas no seu domínio de definição.

Observação 1.4.2 A indistinguibilidade é uma relação de equivalência em $\mathcal{M}$. 
Exemplo 1.4.1 Sejam $\mathcal{M}=\mathbb{R}, \mathcal{U}=\mathbb{R}$ e consideremos o sistema:

$$
\begin{aligned}
\dot{x} & =u \\
y_{1} & =\cos x \\
y_{2} & =\sin x
\end{aligned}
$$

Com $x(0)=x_{0}$ os estados $x_{0}$ e $x_{k}$ são indistinguiveis, onde $x_{k}=x_{0}+2 k \pi$ para quaisquer $x_{0}$ e $k \in \mathcal{Z}$.

Definição 1.4.2 O sistema (1) é dito observável quando $x_{1} \mathcal{I} x_{2}$ implica que $x_{1}=x_{2}$. Equivalentemente se $\mathcal{I}(x)=\left\{x_{0} \in \mathcal{M}: x_{0} \mathcal{I} x\right\}=\{x\}$ para todo $x \in \mathcal{M}$, onde $\mathcal{I}(x) e ́$ a classe de equivalência de $x$.

A observabilidade é um conceito global podemos percorrer uma considerável distância ou um longo tempo para distinguir entre pontos de $\mathcal{M}$. Portanto introduziremos um conceito local que é mais forte que observabilidade.

Definição 1.4.3 Seja $\mathcal{V} \subset \mathcal{M}$ um conjunto aberto tal que $x_{1}, x_{2} \in \mathcal{V}$. Diremos que $x_{1}$ e $x_{2}$ são $\mathcal{V}$-indistinguíveis denotado por $x_{1} \mathcal{I}_{\mathcal{V}} x_{2}$, se para todo controle admissível $u:[0, T] \longrightarrow \mathcal{U}, T>0$ arbitrário, com a propriedade de que as soluçôes $x\left(t, 0, x_{1}, u\right)$ e $x\left(t, 0, x_{2}, u\right)$ ambas permaneçam em $\mathcal{V}$ para $t \leq T$, as funçôes de saída $y\left(t, 0, x_{1}, u\right)$ e $y\left(t, 0, x_{2}, u\right)$ são as mesmas para $t \leq T$, no domínio de definiçâo.

$\mathcal{V}$-indistinguibilidade não é, em geral, uma relação de equivalência em $\mathcal{V}$, falha a transitividade, como conseqüência de que o sistema restrito a $\mathcal{V}$ não necesariamente é completo, como é mostrado em [21]. Entretanto, podemos definir no sistema (1) o que entenderemos por localmente observável.

Definição 1.4.4 O sistema (1) é localmente observável em $x_{0}$ se para toda vizinhança aberta $\mathcal{V}$ de $x_{0}$ a relação $x_{0} \mathcal{I} \mathcal{V} x_{1}$ implica $x_{0}=x_{1}$ o que é o mesmo que a classe de $x_{0}, \mathcal{I}_{\mathcal{V}}\left(x_{0}\right)=\left\{x_{0}\right\}$. Se o sistema é localmente observável em cada $x_{0} \in \mathcal{M}$ então diremos que é localmente observável.

Em [16] nós encontramos com outra definição de localmente observável, a qual nós definiremos como localmente fracamente observável, seguindo [7]

$\mathrm{Na}$ prática nos interessa saber se $x_{0}$ é distinguivel desde uma certa vizinhança, por isto definamos o que entenderemos por fracamente observável. 
Definição 1.4.5 O sistema (1) é fracamente observável em $x_{0}$ se existe uma vizinhança $\mathcal{V}$ de $x_{0}$ tal que $\mathcal{I}\left(x_{0}\right) \cap \mathcal{V}=\left\{x_{0}\right\}$. O sistema é fracamente observável se é fracamente observável em cada $x_{0} \in \mathcal{M}$.

Em [16] Nijmeijer define fracamente observável como localmente observável.

Uma vez mais pode ser necessário percorrer uma considerável distância de $\mathcal{V}$ para distinguir pontos de $\mathcal{V}$, vejamos um conceito local.

Definição 1.4.6 O sistema (1) é localmente fracamente observável em $x_{0}$, se existe uma vizinhança aberta $\mathcal{W}$ de $x_{0}$ tal que para toda vizinhança aberta $\mathcal{V}$ de $x_{0}, \mathcal{V} \subset$ $\mathcal{W}, \mathcal{I}_{\mathcal{V}}\left(x_{0}\right)=\left\{x_{0}\right\}$. Diremos que o sistema é localmente fracamente observável se a igualdade estiver satisfeita para todo $x_{0} \in \mathcal{M}$.

Observação 1.4.3 Intuitivamente o sistema (1) é localmente fracamente observável se podemos instantaneamente distinguir cada ponto desde suas vizinhanças. A vantagem desta definição é que pode ser conferida com um simples teste algébrico.

Dado o sistema (1) é fácil ver que temos as seguintes implicações:
(1)localmente observável
$\Downarrow$
$\Longrightarrow$ (1)observável
$\Downarrow$
(1)localmente fracamente observável $\Longrightarrow$ (1)fracamente observável

As seguintes definições nos fornecem um teste simple para saber quando o sistema (1) é localmente fracamente observável.

Definição 1.4.7 $O$ espaço de observação $\mathcal{O}$ do sistema (1) é o espaço vetorial (sobre $\mathbb{R}$ ) das funções reais em $\mathcal{M}$ que contém $h_{1}, \ldots, h_{p}$ e todas as repetidas derivadas de Lie:

$$
\mathcal{L}_{X_{1}} \mathcal{L}_{X_{2}} \ldots \mathcal{L}_{X_{k}} h_{j} \quad 1 \leq j \leq p, \quad k=1,2, \ldots
$$

com $X_{i}$ no conjunto $\left\{f, g_{1}, \ldots, g_{m}\right\}, 1 \leq i \leq k$

Vejamos uma caracterização equivalente de $\mathcal{O}$ que é dada em [7], mediante a seguinte proposição.

Proposição 1.4.1 O espaço de observação $\mathcal{O}$ é dado pelo espaço vetorial das funções em $\mathcal{M}$ geradas por $h_{1}, \ldots, h_{p}$ e todas as iteradas derivadas de Lie.

$$
\mathcal{L}_{Z_{1}} \mathcal{L}_{Z_{2}} \ldots \mathcal{L}_{Z_{k}} h_{j}, \quad 1 \leq j \leq p, \quad k=1,2, \ldots
$$


onde $Z_{i}, 1 \leq i \leq k$ é da forma

$$
Z_{i}(x)=f(x)+\sum_{j=1}^{m} g_{j}(x) u_{j}^{i}
$$

para algum $u^{i}=\left(u_{1}^{i}, \ldots, u_{m}^{i}\right) \in \mathcal{U}, Z_{i} \in \mathcal{F}$

Demonstração: Usaremos a seguintes propriedades:

$$
\begin{gathered}
\mathcal{L}_{\left(X_{1}+X_{2}\right)} H=\mathcal{L}_{X_{1}} H+\mathcal{L}_{X_{2}} H \\
\mathcal{L}_{X}\left(H_{1}+H_{2}\right)=\mathcal{L}_{X} H_{1}+\mathcal{L}_{X} H_{2}
\end{gathered}
$$

onde $X, X_{1}, X_{2}$ são campos vetoriais e $H, H_{1}, H_{2}$ funções definidas em $\mathcal{M}$. A prova é por indução sobre $\mathrm{k}$

seja $k=1$ temos que $Z_{1}=f(x)+\sum_{j=1}^{m} g_{j}(x) u_{j}^{1}$

$$
\begin{aligned}
\mathcal{L}_{Z_{1}} h_{j} & =\mathcal{L}\left(f(x)+\sum_{j=1}^{m} g_{j}(x) u_{j}^{1}\right)^{h_{j}} \\
& =\mathcal{L}_{f(x)} h_{j}+\mathcal{L}\left(\sum_{j=1}^{m} g_{j}(x) u_{j}^{1}\right)^{h_{j}}
\end{aligned}
$$

esta soma está contida en $\mathcal{O}$. Portanto $\mathcal{L}_{Z_{1}} h_{j}$ está contida em $\mathcal{O}$. Agora provaremos que $\mathcal{L}_{Z_{1}} \mathcal{L}_{Z_{2}} \ldots \mathcal{L}_{Z_{k}} h_{j}$ está em $\mathcal{O}$

$$
\begin{aligned}
\mathcal{L}_{Z_{1}} \mathcal{L}_{Z_{2}} \ldots \mathcal{L}_{Z_{k}} h_{j} & =\mathcal{L}_{Z_{1}} \mathcal{L}_{Z_{2}} \ldots \mathcal{L}_{Z_{k-1}} \mathcal{L}\left(f(x)+\sum_{j=1}^{m} g_{j}(x) u_{j}^{k}\right)^{h_{j}} \\
& =\mathcal{L}_{Z_{1}} \mathcal{L}_{Z_{2} \ldots \mathcal{L}_{Z_{k-1}}\left(\mathcal{L}_{f(x)} h_{j}+\mathcal{L}\right.}\left(\sum_{j=1}^{m} g_{j}(x) u_{j}^{k}\right)^{\left.h_{j}\right)} \\
& =\mathcal{L}_{Z_{1}} \mathcal{L}_{Z_{2}} \ldots \mathcal{L}_{Z_{k-1}}\left(\mathcal{L}_{f(x)} h_{j}\right)+\mathcal{L}_{Z_{1}} \mathcal{L}_{Z_{2}} \ldots \mathcal{L}_{Z_{k-1}}\left(\mathcal{L}\left(\sum_{j=1}^{m} g_{j}(x) u_{j}^{k}\right)^{\left.h_{j}\right)}\right.
\end{aligned}
$$

Pela hipótese de indução temos que esta soma pertence a $\mathcal{O}$. Portanto temos que

$$
\mathcal{L}_{Z_{1}} \ldots \mathcal{L}_{Z_{k}} h_{j} \quad \text { está em } \mathcal{O} \text {. }
$$


Por outro lado, fazendo $f=Z_{i}$ para $u^{i}=0$ e $g_{j}=\frac{1}{2}\left(f+g_{j}\right)-\frac{1}{2}\left(f-g_{j}\right)$ todo campo vetorial $f, g_{1}, \ldots, g_{m}$ definido em $\mathcal{M}$ é uma combinação linear dos $Z_{i}$. Isto demonstra a proposição.

Podemos concluir que o espaço de observação $\mathcal{O}$ contém as funções de saída e todas as derivadas da função de saída ao longo de sistema de trajetórias.

Proposição 1.4.2 $O$ espaço de observação $\mathcal{O}$ não muda se trocamos os campos vetoriais $X_{i}, \quad 1 \leq i \leq k$ em $\mathcal{L}_{X_{1}} \mathcal{L}_{X_{2}} \ldots \mathcal{L}_{X_{k}} h_{j} \quad 1 \leq j \leq p \quad k=1,2, \ldots$ por elementos $d a$ álgebra acessivei $\mathcal{C}$.

Demonstração: Um elemento da álgebra acessível $\mathcal{C}$ de comprimento $k$ é

$$
\left[X_{k},\left[X_{k-1},\left[\ldots,\left[X_{1}, X_{0}\right] \ldots\right]\right]\right]
$$

Apliquemos indução ao comprimento. Tomemos un elemento de comprimento $k=1$. Pela definição de colchete de Lie

$$
\mathcal{L}_{\left[X_{1}, X_{0}\right]} h_{i}=\mathcal{L}_{X_{1}}\left(\mathcal{L}_{X_{0}} h_{i}\right)-\mathcal{L}_{X_{0}}\left(\mathcal{L}_{X_{1}} h_{i}\right),
$$

onde $X_{0}, X_{1} \in\left\{f, g_{1}, \ldots, g_{m}\right\}$, decorre da Definição (1.4.7), que esta diferença pertence a $\mathcal{O}$, portanto

$$
\mathcal{L}_{\left[X_{1}, X_{0}\right]} h_{i} \in \mathcal{O}, \quad 1 \leq i \leq p .
$$

Suponhamos que é válido para $k-1$, ou seja

$$
\mathcal{L}_{\left[X_{k-1},\left[X_{k-2}, \ldots,\left[X_{0}, X_{1}\right] \ldots\right]\right]} h_{i} \in \mathcal{O}
$$

Onde $X_{j} \in\left\{f, g_{1}, \ldots, g_{m}\right\}, \quad 0 \leq j \leq k-1,1 \leq i \leq p$.

Demonstremos que é válido para $k$. Temos que

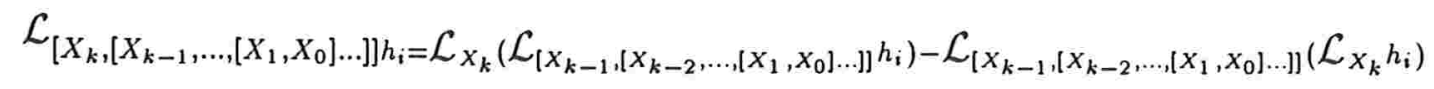

segue da hipótese de indução e da Definição (1.4.7), que

$$
\mathcal{L}\left[X_{k},\left[X_{k-1}, \ldots,\left[X_{1}, X_{0}\right] \ldots\right]\right] h_{i} \in \mathcal{O}
$$

O qual demonstra a proposição.

Observação 1.4.4 E da mesma forma, se $h_{i}$ é substituída por qualquer função $\mathcal{L}_{X_{1}} \mathcal{L}_{X_{2}} \ldots \mathcal{L}_{X_{k}} h_{j}, \quad 1 \leq j \leq p, \quad k=1,2, \ldots X_{i} \in\left\{f, g_{1}, \ldots, g_{m}\right\}$. 
A partir do espaço de observação $\mathcal{O}$ podemos definir a codistribuição de observabilidade denotada por $d \mathcal{O}$, isto é

$$
d \mathcal{O}(q)=\operatorname{Span}\{d H(q) \backslash H \in \mathcal{O}\}, q \in \mathcal{M}
$$

Temos que $d \mathcal{O}$ é gerado por 1-formas exatas.

A derivada de Lie é definida por:

$$
\left.\mathcal{L}_{X_{1}}\left(Y_{1}\right)(x)=\left(\frac{\partial Y_{1}^{t}}{\partial x}(x) X_{1}(x)\right)^{t}-Y_{1}(x) \frac{\partial X_{1}}{\partial x}\right)
$$

onde $Y_{1} \in d \mathcal{O}, X_{1}$ campo vetorial.

Temos a fórmula de Leibnitz:

$$
\mathcal{L}_{X_{1}}\left\langle Y, X_{2}\right\rangle=\left\langle\mathcal{L}_{X_{1}} Y, X_{2}\right\rangle+\left\langle Y,\left[X_{1}, X_{2}\right]\right\rangle
$$

Se $Y=d H$ então $\mathcal{L}_{X}$ c $d$ comutam, isto é

$$
\mathcal{L}_{X}(d H)=d\left(\mathcal{L}_{X}(H)\right)
$$

Logo, segue que a codistribuição $d \mathcal{O}$ é involutiva. Os elementos de $d \mathcal{O}$ são combinações lineares finitas de 1-formas da forma:

$$
d\left(\mathcal{L}_{Z_{1}}\left(\ldots\left(\mathcal{L}_{Z_{k}} h_{i}\right) \ldots\right)\right)=\mathcal{L}_{Z_{1}}\left(\ldots\left(\mathcal{L}_{Z_{k}} d h_{i}\right) \ldots\right)
$$

Onde $Z_{i}$ é definido como na Proposição (1.4.1).

Vejamos agora um teorema que nos ajudará a saber quando um sistema é localmente fracamente observável.

Teorema 1.4.1 Seja o sistema (1) tal que $\operatorname{dim} \mathcal{M}=n$. Se $d \mathcal{O}\left(x_{0}\right)=T_{x_{0}}^{*} \mathcal{M}$, ou seja, $\operatorname{dim} d \mathcal{O}\left(x_{0}\right)=n$ então o sistema é localmente fracamente observável em $x_{0}$.

Demonstração: Se $\operatorname{dim} d \mathcal{O}\left(x_{0}\right)=n$ então existem $n$ funções $H_{1}, \ldots, H_{n} \in \mathcal{O}$ tal que $\left\{d H_{1}\left(x_{0}\right), \ldots, d H_{n}\left(x_{0}\right)\right\}$ são linearmente independentes. Definamos uma transformação $\phi: \mathcal{M} \longrightarrow \mathbb{R}^{n}$ dada por

$$
\phi(x)=\left(H_{1}(x), \ldots, H_{n}(x)\right)^{t},
$$

Sendo o Jacobiano de $\phi$ em $x_{o}$ não-singular, segue que $\phi$ restrita a uma vizinhança aberta $\mathcal{W}$ de $x_{0}$ é injetora. Se $\mathcal{V} \subset \mathcal{W}$ é uma vizinhança aberta de $x_{0}$ e $x_{0} \mathcal{I}_{\mathcal{V}} x_{1}$ para algum $x_{1} \in \mathcal{V}$ então para qualquer $1 \leq i \leq p$ e $k \geq 0$ e para $t_{1}, \ldots, t_{k}$ pequenos temos que:

$$
h_{i}\left(\gamma_{k}^{t_{k}} o \gamma_{k-1}^{t_{k-1}} o \ldots o \gamma_{1}^{t_{1}}\left(x_{0}\right)\right)=h_{i}\left(\gamma_{k}^{t_{k}} o \gamma_{k-1}^{t_{k-1}} o \ldots o \gamma_{1}^{t_{1}}\left(x_{1}\right)\right)
$$


onde $\gamma_{i}^{t_{i}}(x)$ denota o fluxo de $Z_{i}(x)=f(x)+\sum_{j=1}^{m} g_{j}(x) u_{j}^{i} \quad 1 \leq i \leq k$. Diferenciando em ambos os lados com relação a $t_{k}, \ldots, t_{1}$ (nesta ordem) e fazendo $t_{k}=t_{k-1}=\ldots=t_{1}=0$, temos,

$$
\mathcal{L}_{Z_{1}}\left(\mathcal{L}_{\widetilde{Z}_{2}}\left(\ldots \mathcal{L}_{Z_{k}} h_{i}\left(x_{0}\right) \ldots\right)\right)=\mathcal{L}_{Z_{1}}\left(\mathcal{L}_{Z_{2}}\left(\ldots \mathcal{L}_{Z_{k}} h_{i}\left(x_{1}\right) \ldots\right)\right)
$$

Pela Proposição(1.4.1) segue que $H\left(x_{0}\right)=H\left(x_{1}\right)$ para todo $H \in \mathcal{O}$. Em particular, $H_{i}\left(x_{0}\right)=H_{i}\left(x_{1}\right) \quad 1 \leq i \leq n$ e sendo $\phi$ injetora em $\mathcal{W}$, temos que $x_{0}=x_{1}$. Resumindo temos que para toda vizinhança aberta $\mathcal{V}$ de $x_{0}, \mathcal{V} \subset \mathcal{W}$ se $x_{0} \mathcal{I} \mathcal{V} x_{1}$ implica que $x_{0}=x_{1}$, isto é, o sistema é localmente fracamente observável.

Exemplo 1.4.2 Consideremos uma 2-articulação rígida de um robô manipulador ou pêndulo duplo, onde os torques $u_{1}, u_{2}$ aplicados nas juntas representam o controle.

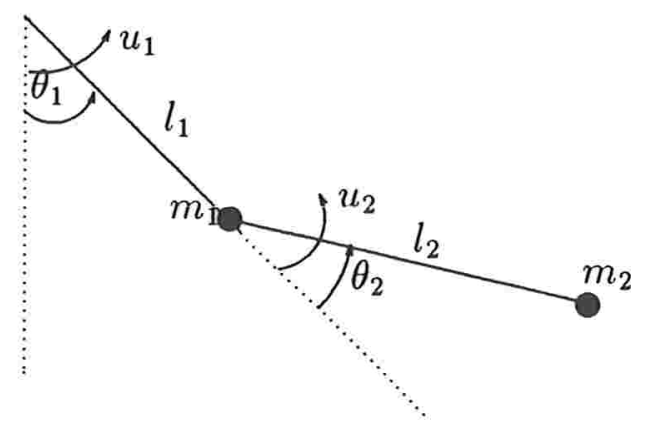

Figura 1.2:

A dinâmica do braço-robô, pode ser obtida via a equação de Euler-Lagrange. Consideremos $\theta=\left(\theta_{1}, \theta_{2}\right)$ e $\dot{\theta}=\left(\dot{\theta}_{1}, \dot{\theta}_{2}\right)$, a função de Lagrange é dada por:

$$
L(\theta, \dot{\theta})=T(\theta, \dot{\theta})-V(\theta)
$$

Onde $T(\theta, \dot{\theta})$ é a energia cinética e $V(\theta)$ a energia potencial. A energia cinética,é

$$
T(\theta, \dot{\theta})=T_{1}(\dot{\theta})+T_{2}(\theta, \dot{\theta})
$$

Onde $T_{1}(\dot{\theta})$ e $T_{2}(\theta, \dot{\theta})$ são a energia cinética das massas $m_{1}$ e $m_{2}$ respectivamente, i.e,

$$
T_{1}=\frac{1}{2} m_{1} v_{1}^{2} \text { e } T_{2}=\frac{1}{2} m_{2} v_{2}^{2} .
$$

Consideremos um deslocamento infinitesimal do pêndulo duplo, caracterizado pelos incrementos $d \theta_{1}$ e $d\left(\theta_{2}+\theta_{1}\right)$ nas coordenadas generalizadas (figura 1.3). A massa $m_{1}$ move-se de $A$ para $A^{\prime}$ e sofre um deslocamento $d s_{1}=l_{1} d \theta$. Portanio

$$
v_{1}^{2}=\left(\frac{d s_{1}}{d t}\right)^{2}=\left(l_{1} \frac{d \theta_{1}}{d t}\right)^{2}=l_{1}^{2} \dot{\theta}_{1}^{2}
$$




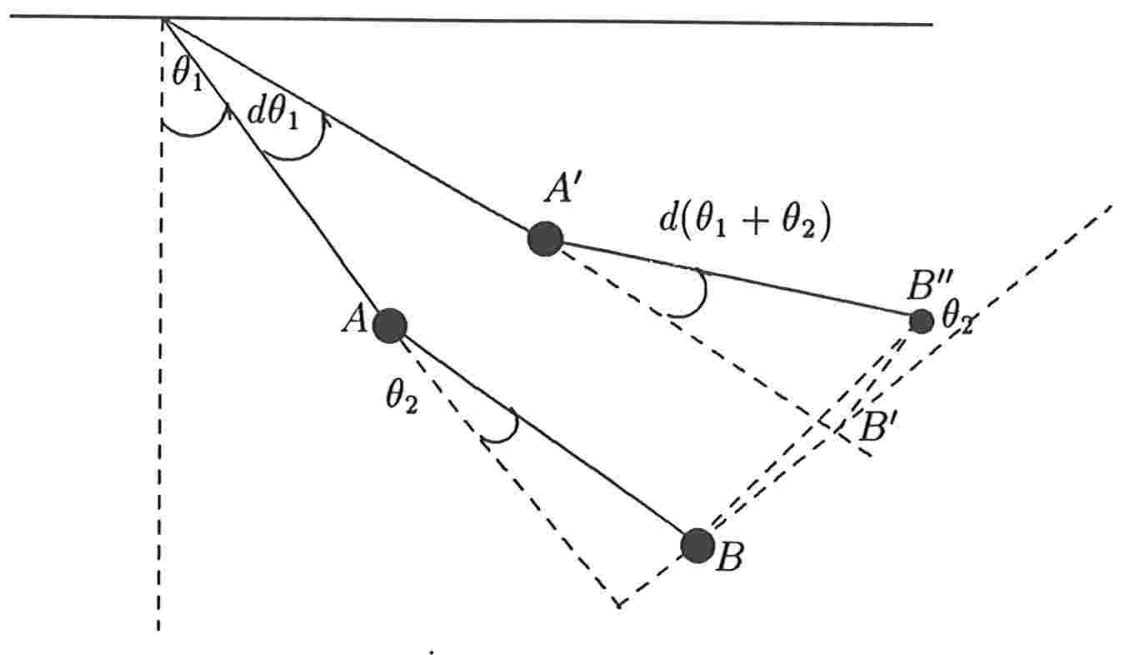

Figura 1.3:

A massa $m_{2}$ move-se de $B$ para $B^{\prime \prime}$ e este deslocamento $d_{2}=B B^{\prime \prime}$. O pêndulo inferior é ainda movimentado em um ângulo $d\left(\theta_{1}+\theta_{2}\right)$ fazendo que o ponto $B^{\prime}$ movase até $B^{\prime \prime}$, com $B^{\prime} B^{\prime \prime}=l_{2} d\left(\theta_{1}+\theta_{2}\right)$. O deslocamento total $B B^{\prime \prime}$ de $m_{2} e^{\prime}$

$$
\begin{aligned}
\left(B B^{\prime \prime}\right)^{2} & =\left(B B^{\prime}\right)^{2}+\left(B^{\prime} B^{\prime \prime}\right)^{2}-2\left(B B^{\prime}\right)\left(B^{\prime} B^{\prime \prime}\right) \cos \left(\pi-\theta_{2}\right) \\
& =\left(B B^{\prime}\right)^{2}+\left(B^{\prime} B^{\prime \prime}\right)^{2}+2\left(B B^{\prime}\right)\left(B^{\prime} B^{\prime \prime}\right) \cos \theta_{2} \\
& =\left(l_{1} d \theta_{1}\right)^{2}+\left(l_{2} d\left(\theta_{1}+\theta_{2}\right)\right)^{2}+2\left(l_{1} d \theta_{1}\right)\left(l_{2} d\left(\theta_{1}+\theta_{2}\right)\right) \cos \theta_{2}
\end{aligned}
$$

dai segue que

$$
v_{2}^{2}=l_{1}^{2} \dot{\theta}^{2}+l_{2}^{2}\left(\dot{\theta_{1}}+\dot{\theta_{2}}\right)^{2}+2 l_{1} l_{2} \dot{\theta}_{1}\left(\dot{\theta_{1}}+\dot{\theta_{2}}\right) \cos \theta_{2}
$$

Portanto temos que

$$
\begin{aligned}
T_{1}(\dot{\theta}) & =\frac{1}{2} m_{1} v_{1}^{2}=\frac{1}{2} m_{1} l_{1}^{2} \dot{\theta}_{1}^{2} \\
T_{2}(\theta, \dot{\theta}) & =\frac{1}{2} m_{2} v_{2}^{2}=\frac{1}{2} m_{2}\left(l_{1}^{2} \dot{\theta}_{1}^{2}+l_{2}^{2}\left(\dot{\theta}_{1}+\dot{\theta}_{2}\right)^{2}+2 l_{1} l_{2} \cos \theta_{2} \dot{\theta}_{1}\left(\dot{\theta_{1}}+\dot{\theta_{2}}\right)\right),
\end{aligned}
$$

e finalmente,

$$
\begin{aligned}
T(\theta, \dot{\theta}) & =T_{1}(\dot{\theta})+T_{2}(\theta, \dot{\theta}) \\
& =\frac{1}{2} m_{1} l_{1}^{2} \dot{\theta}_{1}^{2}+\frac{1}{2} m_{2}\left(l_{1}^{2} \dot{\theta}_{1}{ }^{2}+l_{2}^{2}\left(\dot{\theta_{1}}+\dot{\theta}_{2}\right)^{2}+2 l_{1} l_{2} \cos \theta_{2} \dot{\theta}_{1}\left(\dot{\theta_{1}}+\dot{\theta_{2}}\right)\right)
\end{aligned}
$$

Similarmente a energia potencial $V(\theta)$ é a soma da energia potencial $V_{1}(\theta)$ e $V_{2}(\theta)$ das duas massas.

$$
\begin{aligned}
& V_{1}(\theta)=m_{1} g y_{1}=-m_{1} g l_{1} \cos \theta_{1} \\
& V_{2}(\theta)=m_{2} g y_{2}=-m_{2} g\left(l_{1} \cos \theta_{1}+l_{2} \cos \left(\theta_{1}+\theta_{2}\right)\right),
\end{aligned}
$$


isto é

$$
V(\theta)=V_{1}(\theta)+V_{2}(\theta)=-m_{1} g l_{1} \cos \theta_{1}-m_{2} g\left(l_{1} \cos \theta_{1}+l_{2} \cos \left(\theta_{1}+\theta_{2}\right)\right)
$$

Portanto a função Langrangeana é representada por

$$
\begin{aligned}
L(\theta, \dot{\theta})= & T(\theta, \dot{\theta})-V(\theta) \\
= & \frac{1}{2} m_{1} l_{1}^{2} \dot{\theta}_{1}{ }^{2}+\frac{1}{2} m_{2}\left(l_{1}^{2} \dot{\theta}_{1}{ }^{2}+l_{2}^{2}\left(\dot{\theta_{1}}+\dot{\theta_{2}}\right)^{2}+2 l_{1} l_{2} \cos \theta_{2} \dot{\theta_{1}}\left(\dot{\theta_{1}}+\dot{\theta_{2}}\right)\right) \\
& +m_{1} g l_{1} \cos \theta_{1}+m_{2} g\left(l_{1} \cos \theta_{1}+l_{2} \cos \left(\theta_{1}+\theta_{2}\right)\right) .
\end{aligned}
$$

Agora, calculemos a equação de Euler-Lagrange dada por:

$$
\frac{d}{d t}\left(\frac{\partial L}{\partial \dot{\theta}_{i}}\right)-\frac{\partial L}{\partial \theta_{i}}=u_{i}, \quad i=1,2 .
$$

Temos que:

$$
\begin{aligned}
\frac{\partial L}{\partial \dot{\theta}_{1}}= & m_{1} l_{1}^{2} \dot{\theta}_{1}+m_{2} l_{1}^{2} \dot{\theta}_{1}+m_{2} l_{1} l_{2} \cos \theta_{2}\left(2 \dot{\theta_{1}}+\dot{\theta}_{2}\right)+m_{2} l_{2}^{2}\left(\dot{\theta_{1}}, \dot{\theta_{2}}\right) . \\
\frac{\partial L}{\partial \dot{\theta}_{2}}= & m_{2} l_{2}^{2}\left(\dot{\theta_{1}}+\dot{\theta_{2}}\right)+m_{2} l_{1} l_{2} \cos \theta_{2} \dot{\theta_{1}} . \\
\frac{d}{d t}\left(\frac{\partial L}{\partial \dot{\theta}_{1}}\right)= & \ddot{\theta}_{1}\left(m_{1} l_{1}^{2}+m_{2} l_{1}^{2}+m_{2} l_{2}^{2}+2 m_{2} l_{1} l_{2} \cos \theta_{2}\right)+\ddot{\theta}_{2}\left(m_{2} l_{2}^{2}+m_{2} l_{1} l_{2} \cos \theta_{2}\right) \\
& -2 m_{2} l_{1} l_{2} \sin \theta_{2} \dot{\theta_{2}} \dot{\theta_{1}}-m_{2} l_{1} l_{2} \sin \theta_{2} \dot{\theta}_{2}{ }^{2} . \\
\frac{d}{d t}\left(\frac{\partial L}{\partial \dot{\theta}_{2}}\right)= & \ddot{\theta}_{1}\left(m_{2} l_{2}^{2}+m_{2} l_{1} l_{2} \cos \theta_{2}\right)+\ddot{\theta}_{2} m_{2} l_{2}^{2}-m_{2} l_{1} l_{2} \sin \theta_{2} \dot{\theta_{2}} \dot{\theta_{1}} . \\
\frac{\partial L}{\partial \theta_{1}}= & -m_{1} g l_{1} \sin \theta_{1}-m_{2} g l_{1} \sin \theta_{1}-m_{2} g l_{2} \sin \left(\theta_{1}+\theta_{2}\right) . \\
\frac{\partial L}{\partial \theta_{2}}= & -m_{2} l_{1} l_{2} \sin \theta_{2}\left(\dot{\theta}_{1}{ }^{2}+\dot{\theta_{1}} \dot{\theta_{2}}\right)-m_{2} g l_{2} \sin \left(\theta_{1}+\theta_{2}\right) .
\end{aligned}
$$

Substituindo temos:

$$
\begin{aligned}
\frac{d}{d t}\left(\frac{\partial L}{\partial \dot{\theta}_{1}}\right)-\frac{\partial L}{\partial \theta_{1}}= & \ddot{\theta}_{1}\left(m_{1} l_{1}^{2}+m_{2} l_{1}^{2}+m_{2} l_{2}^{2}+2 m_{2} l_{1} l_{2} \cos \theta_{2}\right)+\ddot{\theta}_{2}\left(m_{2} l_{2}^{2}+m_{2} l_{1} l_{2} \cos \theta_{2}\right) \\
& -2 m_{2} l_{1} l_{2} \sin \theta_{2} \dot{\theta}_{2} \dot{\theta}_{1}-m_{2} l_{1} l_{2} \sin \theta_{2} \dot{\theta}_{2}{ }^{2}+m_{1} g l_{1} \sin \theta_{1}+m_{2} g l_{1} \sin \theta_{1} \\
& +m_{2} g l_{2} \sin \left(\theta_{1}+\theta_{2}\right) . \\
\frac{d}{d t}\left(\frac{\partial L}{\partial \dot{\theta}_{2}}\right)-\frac{\partial L}{\partial \theta_{2}}= & \ddot{\theta}_{1}\left(m_{2} l_{2}^{2}+m_{2} l_{1} l_{2} \cos \theta_{2}\right)+\ddot{\theta}_{2} m_{2} l_{2}^{2}+m_{2} l_{1} l_{2} \sin \theta_{2} \dot{\theta}_{1}{ }^{2} \\
& +m_{2} g l_{2} \sin \left(\theta_{1}+\theta_{2}\right) .
\end{aligned}
$$


Escrevendo em forma vetorial temos:

$$
\begin{gathered}
\left(\begin{array}{cc}
m_{1} l_{1}^{2}+m_{2} l_{1}^{2}+m_{2} l_{2}^{2}+2 m_{2} l_{1} l_{2} \cos \theta_{2} & m_{2} l_{2}^{2}+m_{2} l_{1} l_{2} \cos \theta_{2} \\
m_{2} l_{2}^{2}+m_{2} l_{1} l_{2} \cos \theta_{2} & m_{2} l_{2}^{2}
\end{array}\right)\left(\begin{array}{c}
\ddot{\theta_{1}} \\
\ddot{\theta_{2}}
\end{array}\right) \\
+\left(\begin{array}{c}
-2 m_{2} l_{1} l_{2} \sin \theta_{2} \dot{\theta_{1}} \dot{\theta_{2}}-m_{2} l_{1} l_{2} \sin \theta_{2} \dot{\theta}_{2}{ }^{2} \\
m_{2} l_{1} l_{2} \sin \theta_{2} \dot{\theta}_{1}{ }^{2}
\end{array}\right) \\
+\left(\begin{array}{c}
m_{1} g l_{1} \sin \theta_{1}+m_{2} g l_{1} \sin \theta_{1}+m_{2} g l_{2} \sin \left(\theta_{1}+\theta_{2}\right) \\
m_{2} g l_{2} \sin \left(\theta_{1}+\theta_{2}\right)
\end{array}\right)=\left(\begin{array}{l}
u_{1} \\
u_{2}
\end{array}\right) .
\end{gathered}
$$

Isto é

$$
A(\theta) \ddot{\theta}+B(\theta, \dot{\theta})+C(\theta)=u
$$

onde $\ddot{\theta}=\left(\ddot{\theta}_{1}, \ddot{\theta}_{2}\right) \quad u=\left(u_{1}, u_{2}\right) e$

$$
\begin{aligned}
A(\theta) & =\left(\begin{array}{cc}
m_{1} l_{1}^{2}+m_{2} l_{1}^{2}+m_{2} l_{2}^{2}+2 m_{2} l_{1} l_{2} \cos \theta_{2} & m_{2} l_{2}^{2}+m_{2} l_{1} l_{2} \cos \theta_{2} \\
m_{2} l_{2}^{2}+m_{2} l_{1} l_{2} \cos \theta_{2} & m_{2} l_{2}^{2}
\end{array}\right) \\
B(\theta, \dot{\theta}) & =\left(\begin{array}{c}
-m_{2} l_{1} l_{2} \sin \theta_{2} \dot{\theta_{2}}\left(2 \dot{\theta_{1}}+\dot{\theta_{2}}\right) \\
m_{2} l_{1} l_{2} \sin \theta_{2} \dot{\theta}_{1}{ }^{2}
\end{array}\right) \\
C(\theta) & =\left(\begin{array}{c}
m_{1} g l_{1} \sin \theta_{1}+m_{2} g l_{1} \sin \theta_{1}+m_{2} g l_{2} \sin \left(\theta_{1}+\theta_{2}\right) \\
m_{2} g l_{2} \sin \left(\theta_{1}+\theta_{2}\right)
\end{array}\right),
\end{aligned}
$$

Onde $C(\theta)$ representa a força gravitacional e $B(\theta, \dot{\theta})$ é a força centrípeta. Temos que

$$
\begin{aligned}
\operatorname{Det} A(\theta) & =\left|\begin{array}{cc}
m_{1} l_{1}^{2}+m_{2} l_{1}^{2}+m_{2} l_{2}^{2}+2 m_{2} l_{1} l_{2} \cos \theta_{2} & m_{2} l_{2}^{2}+m_{2} l_{1} l_{2} \cos \theta_{2} \\
m_{2} l_{2}^{2}+m_{2} l_{1} l_{2} \cos \theta_{2} & m_{2} l_{2}^{2}
\end{array}\right| \\
& =m_{1} m_{2} l_{1}^{2} l_{2}^{2}+m_{2}^{2} l_{1}^{2} l_{2}^{2}-m_{2}^{2} l_{1}^{2} l_{2}^{2} \cos ^{2} \theta_{2} \\
& =m_{1} m_{2} l_{1}^{2} l_{2}^{2}+m_{2}^{2} l_{1}^{2} l_{2}^{2}\left(1-\cos ^{2} \theta_{2}\right) \\
& =m_{1} m_{2} l_{1}^{2} l_{2}^{2}+m_{2}^{2} l_{1}^{2} l_{2}^{2} \sin ^{2} \theta_{2}
\end{aligned}
$$

Portanto $\operatorname{det} A(\theta)>0$ para todo $\theta$ o que implica que $A(\theta)$ é invertível. Portanto: 


$$
\begin{aligned}
A(\theta) \ddot{\theta}+B(\theta, \dot{\theta})+C(\theta) & =u \\
A(\theta) \ddot{\theta} & =-B(\theta, \dot{\theta})-C(\theta)+u \\
\ddot{\theta} & =-A^{-1}(\theta) B(\theta, \dot{\theta})-A^{-1}(\theta) C(\theta)+A^{-1}(\theta) u
\end{aligned}
$$

esta equação descreve a dinâmica de uma 2-articulação do braço-robô, a qual é um sistema de controle não-linear com espaço de estado

$$
\left(\theta_{1}, \dot{\theta_{1}}, \theta_{2}, \dot{\theta_{2}}\right) \in S^{1} \times \mathbb{R} \times S^{1} \times \mathbb{R} \cong T S^{1} \times T S^{1} .
$$

Uma função de saída poderia ser as coordenadas cartesianas do ponto final, é preferivel aos ângulos $\theta_{1}$ e $\theta_{2}$ entre as conexões (figura 1.4).

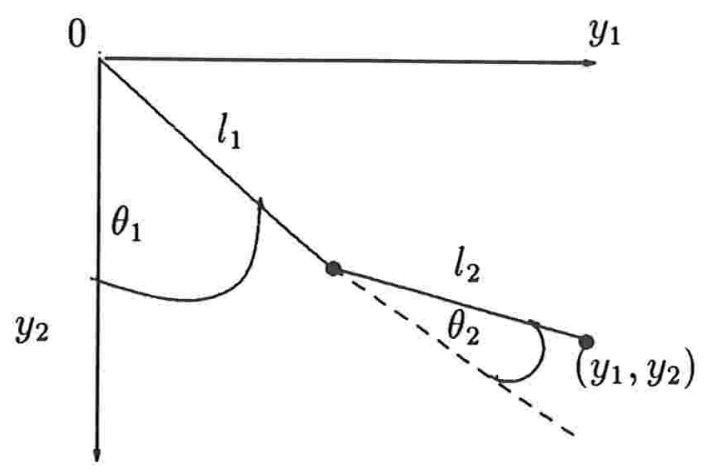

Figura 1.4:

Se denotamos as coordenadas cartesianas do ponto final como $y_{1}, y_{2}$, temos que a transformação de saída vem dada por

$$
\begin{aligned}
& y_{1}=l_{1} \sin \theta_{1}+l_{2} \sin \left(\theta_{1}+\theta_{2}\right) \\
& y_{2}=l_{1} \cos \theta_{1}+l_{2} \cos \left(\theta_{1}+\theta_{2}\right)
\end{aligned}
$$

Isto é chamado direção cinemática do braço-robô. Na prática é importante conhecer os ângulos $\theta_{1}, \theta_{2}$ quando a posição final $\left(y_{1}, y_{2}\right)$ é dada, vejamos como vem dada a expressão. Calculemos o Jacobiano da transformação de saída 


$$
\begin{aligned}
\mathcal{J}\left(y\left(\theta_{1}, \theta_{2}\right)\right) & =\left(\begin{array}{cc}
l_{1} \cos \theta_{1}+l_{2} \cos \left(\theta_{1}+\theta_{2}\right) & l_{2} \cos \left(\theta_{1}+\theta_{2}\right) \\
-l_{1} \sin \theta_{1}-l_{2} \sin \left(\theta_{1}+\theta_{2}\right) & -l_{2} \sin \left(\theta_{1}+\theta_{2}\right)
\end{array}\right) \\
\operatorname{Det} \mathcal{J}\left(\theta_{1}, \theta_{2}\right) & =\left|\begin{array}{cc}
\left.l_{1} \cos \theta_{1}+l_{2} \cos \theta_{1}+\theta_{2}\right) & l_{2} \cos \left(\theta_{1}+\theta_{2}\right) \\
-l_{1} \sin \theta_{1}+l_{2} \sin \left(\theta_{1}+\theta_{2}\right) & -l_{2} \sin \left(\theta_{1}+\theta_{2}\right)
\end{array}\right| \\
& =-l_{1} l_{2} \cos \theta_{1} \sin \left(\theta_{1}+\theta_{2}\right)+l_{1} l_{2} \sin \theta_{1} \cos \theta_{1}+\theta_{2} \\
& =-l_{1} l_{2} \sin \theta_{1} .
\end{aligned}
$$

Sabemos que $\sin \theta_{2}=0$ se $\theta_{2}=k \pi, k \in \mathcal{Z}$. Portanto posto $\mathcal{J}\left(y\left(\theta_{1}, \theta_{2}\right)\right)=2$ para $\theta_{2} \neq k \pi, k \in Z$. Aplicando o Teorema da Função Inversa encontraremos $\theta_{1}, \theta_{2}$ em função $\left(y_{1}, y_{2}\right)$, o qual garante que podemos encontrar os valores de $\theta_{1}, \theta_{2}$ dado o ponto final $\left(y_{1}, y_{2}\right)$. temos

Para simplificar os cálculos assumimos que $m_{1}=m_{2}=1, l_{1}=l_{2}=1$. Portanto

$$
\begin{aligned}
A(\theta) & =\left(\begin{array}{cc}
3+2 \cos \theta_{2} & 1+\cos \theta_{2} \\
1+\cos \theta_{2} & 1
\end{array}\right), \\
\operatorname{Det}(A(\theta)) & =1+\sin ^{2} \theta_{2} \\
A^{-1}(\theta) & =\frac{1}{1+\sin ^{2} \theta_{2}}\left(\begin{array}{cc}
1 & -\left(1+\cos \theta_{2}\right) \\
-\left(1+\cos \theta_{2}\right) & \left(3+2 \cos \theta_{2}\right)
\end{array}\right) .
\end{aligned}
$$

Portanto

$$
\begin{aligned}
\ddot{\theta}=-A^{-1}(\theta) B(\theta, \dot{\theta})-A^{-1}(\theta) C(\theta)+A^{-1}(\theta) u \\
=\frac{-1}{1+\sin ^{2} \theta_{2}}\left(\begin{array}{c}
-\sin \theta_{2} \dot{\theta_{2}}\left(2 \dot{\theta_{1}}+\dot{\theta_{2}}\right)-\left(1+\cos \theta_{2}\right) \sin \theta_{2} \dot{\theta}_{1}^{2} \\
+2 g \sin \theta_{1}-g \cos \theta_{2} \sin \left(\theta_{1}+\theta_{2}\right) \\
\sin \theta_{2} \dot{\dot{\theta}_{2}}\left(2 \dot{\theta_{1}}+\dot{\theta_{2}}\right)+\sin \theta_{2} \cos \theta_{2} \dot{\theta_{2}}\left(2 \dot{\theta_{1}}+\dot{\theta}_{2}\right) \\
+\sin \theta_{2} \dot{\theta}_{1}^{2}\left(3+2 \sin \theta_{2}\right)-2 g \sin \theta_{1}\left(1+\cos \theta_{2}\right) \\
+g \sin \left(\theta_{1}+\theta_{2}\right)\left(2+\cos \theta_{2}\right)
\end{array}\right) \\
+\left(\begin{array}{cc}
-\left(1+\cos \theta_{2}\right) & \left(3+2 \cos \theta_{2}\right)
\end{array}\right)\left(\begin{array}{c}
u_{1} \\
u_{2}
\end{array}\right) .
\end{aligned}
$$


Temos como estado o vetor $x=\left(\theta_{1}, \theta_{2}, \dot{\theta}_{1}, \dot{\theta}_{2}\right) \in T\left(S^{1} \times S^{1}\right)$, tal que $\theta_{1}, \theta_{2}$ são os ângulos definidos no círculo unitário $S^{1}$. O sistema é dado.

$$
\begin{aligned}
& \frac{d}{d t}\left(\begin{array}{c}
\theta_{1} \\
\theta_{2} \\
\dot{\theta_{1}} \\
\dot{\theta_{2}}
\end{array}\right)=\left(\begin{array}{c}
\dot{\theta}_{1} \\
\dot{\theta_{2}} \\
\frac{-1}{1+\sin ^{2} \theta_{2}}\left(-\sin \theta_{2} \dot{\theta_{2}}\left(2 \dot{\theta_{1}}+\dot{\theta}_{2}\right)-\left(1+\cos \theta_{2}\right) \sin \theta_{2} \dot{\theta}_{1}{ }^{2}\right. \\
\left.+2 g \sin \theta_{1}-g \cos \theta_{2} \sin \left(\theta_{1}+\theta_{2}\right)\right) \\
\frac{-1}{1+\sin ^{2} \theta_{2}}\left(\sin \theta_{2} \dot{\theta}_{2}\left(2 \dot{\theta_{1}}+\dot{\theta_{2}}\right)+\sin \theta_{2} \cos \theta_{2} \dot{\theta_{2}}\left(2 \dot{\theta_{1}}+\dot{\theta_{2}}\right)\right. \\
+\sin \dot{\theta}_{2} \dot{\theta}_{1}{ }^{2}\left(3+2 \cos \theta_{2}\right)-2 g \sin \theta_{1}\left(1+\cos \theta_{2}\right) \\
\left.+g \sin \left(\theta_{1}+\theta_{2}\right)\left(2+\cos \theta_{2}\right)\right)
\end{array}\right) \\
& +\frac{1}{1+\sin ^{2} \theta_{2}}\left(\begin{array}{cl}
0 & 0 \\
0 & 0 \\
1 & -\left(1+\cos \theta_{2}\right) \\
-\left(1+\cos \theta_{2}\right) & \left(3+2 \cos \theta_{2}\right)
\end{array}\right)\left(\begin{array}{l}
u_{1} \\
u_{2}
\end{array}\right) .
\end{aligned}
$$

De onde temos os seguintes campos vetoriais que :

$$
\begin{aligned}
f\left(\theta_{1}, \theta_{2}, \dot{\theta_{1}}, \dot{\theta_{2}}\right)= & \dot{\theta_{1}} \frac{\partial}{\partial \theta_{1}}+\dot{\theta_{2}} \frac{\partial}{\partial \theta_{2}}-\frac{1}{1+\sin ^{2} \theta_{2}}\left[-\sin \theta_{2} \dot{\theta_{2}}\left(2 \dot{\theta_{1}}+\dot{\theta_{2}}\right)\right. \\
& -\left(1+\cos \theta_{2}\right) \sin \theta_{2} \dot{\theta}_{1}^{2}+2 g \sin \theta_{1}-g \cos \theta_{2} \sin \left(\theta_{1}+\theta_{2}\right) \frac{\partial}{\partial \dot{\theta}_{1}} \\
& -\frac{1}{1+\sin ^{2} \theta_{2}}\left[\sin \theta_{2} \dot{\theta_{2}}\left(2 \dot{\theta_{1}}+\dot{\theta_{2}}\right)+\sin \theta_{2} \cos \theta_{2} \dot{\theta_{2}}\left(2 \dot{\theta_{1}}+\dot{\theta_{2}}\right)\right. \\
& +\sin \theta_{2} \dot{\theta}_{1}^{2}\left(3+2 \cos \theta_{2}\right)-2 g \sin \theta_{1}\left(1+\cos \theta_{2}\right) \\
& \left.+g \sin \left(\theta_{1}+\theta_{2}\right)\left(2+\cos \theta_{2}\right)\right] \frac{\partial}{\partial \dot{\theta}_{2}} \\
g_{1}\left(\theta_{1}, \theta_{2}, \dot{\theta_{1}}, \dot{\theta_{2}}\right)= & 0 \frac{\partial}{\partial \theta_{1}}+0 \frac{\partial}{\partial \theta_{2}}+\frac{1}{1+\sin ^{2} \theta_{2}} \frac{\partial}{\partial \dot{\theta}_{1}}-\frac{\left(1+\cos \theta_{2}\right)}{1+\cos ^{2} \theta_{2}} \frac{\partial}{\partial \dot{\theta}_{2}} \\
g_{2}\left(\theta_{1}, \theta_{2}, \dot{\theta_{1}}, \dot{\theta_{2}}\right)= & 0 \frac{\partial}{\partial \theta_{1}}+0 \frac{\partial}{\partial \theta_{2}}-\frac{\left(1+\cos ^{2} \theta_{2}\right)}{1+\sin ^{2} \theta_{2}} \frac{\partial}{\partial \dot{\theta}_{1}}+\frac{\left(3+2 \cos \theta_{2}\right)}{1+\sin ^{2} \theta_{2}} \frac{\partial}{\partial \dot{\theta}_{2}}
\end{aligned}
$$


Encontremos à álgebra fortemente acessivel $\mathcal{C}_{0}$, temos que

$$
\begin{aligned}
& {\left[f, g_{1}\right]=\left(\begin{array}{c}
\frac{-1}{1+\sin ^{2} \theta_{2}} \\
\frac{1+\cos \theta_{2}}{1+\sin ^{2} \theta_{2}} \\
0 \\
{\left[f, g_{2}\right]=\left(\begin{array}{c}
\frac{1+\cos \theta_{2}}{1+\sin ^{2} \theta_{2}} \\
\frac{-\left(3+2 \cos \theta_{2}\right)}{1+\sin ^{2} \theta_{2}} \\
\frac{\sin \theta_{2}\left(2 \dot{\theta_{1}}+\dot{\theta_{2}}\right)\left(2-\cos ^{2} \theta_{2}\right)}{\left(1+\sin ^{2} \theta_{2}\right)^{2}}
\end{array}\right)} \\
\frac{\sin \theta_{2}\left(\cos ^{2} \theta_{2}-2\right)\left(2 \dot{\theta_{1}}+\dot{\theta_{2}}\right)}{\left(1+\sin ^{2} \theta_{2}\right)^{2}} \\
0
\end{array}\right)}
\end{aligned}
$$

Portanto $\mathcal{C}_{0}=\left\{g_{1}, g_{2},\left[f, g_{1}\right],\left[f, g_{2}\right]\right\} e$

$$
\operatorname{dim}\left(\begin{array}{lll}
g_{1} & g_{2} & {\left[f, g_{1}\right]}
\end{array}\left[f, g_{2}\right]\right)=4
$$

Portanto $\operatorname{dim} C_{0}(x)=4$ e como $C_{0}(x) \subset T_{x}\left(T\left(S^{1} \times S^{1}\right)\right)$

$$
C_{0}(x)=T_{x}\left(T\left(S^{1} \times S^{1}\right)\right)
$$

Portanto por Teorema (1.3.2) temos que o sistema é fortemente acessível

Exemplo 1.4.3 As equações que descrevem a atitude de um satelite, são basicamente de rotação de um corpo rígido com termos extras descrevendo os efeitos dos torques de controle. Portanto pode-se separar as equações em equações da Cinemática relacionadas à posição angular com a velocidade angular e as equações da Dinâmica descrevendo a evolução da velocidade angular ou equivalentemente, o momento angular.

1) Equações da Cinemática.

Representaremos as equaçôes Cinemática em dois sentidos um global e outro local. Se precisarmos uma descrição global do problema, descreveremos a posição angular mediante a matriz de rotação $R, R$ transforma um conjunto inercialmente fixo de eixos ortonormais $e_{1}, e_{2}, e_{3}$ em um conjunto ortonormal de eixos $r_{1}, r_{2}, r_{3}$ (com a 
mesma orientação que $\left.e_{1}, e_{2}, e_{3}\right)$, os quais são fixados na astronave e tem como origem o centro de masa da astronave (figura 1.5).

$$
R e_{i}=r_{i} \quad i=1,2,3 .
$$

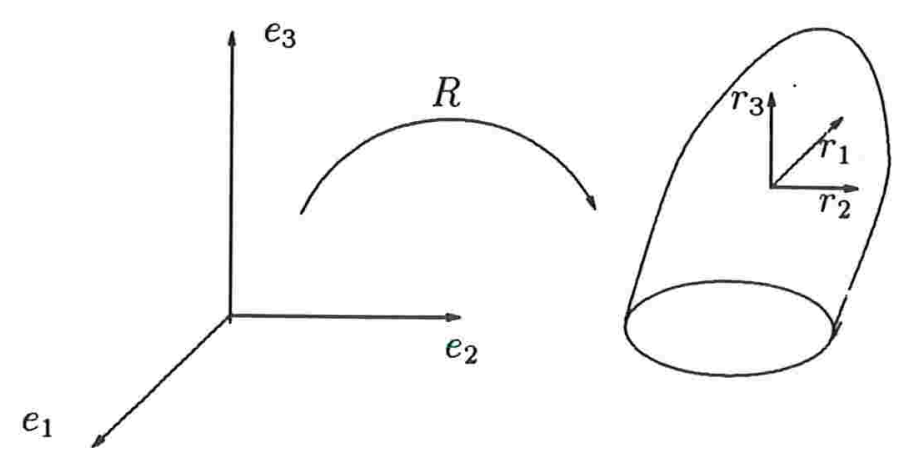

Figura 1.5:

Podemos descrever a posição de um corpo rígido em relação a um conjunto inercial de eixos $e_{1}, e_{2}, e_{3} \in \mathbb{R}^{3}$ pela matriz,

$$
R=\left(\begin{array}{ccc}
r_{1} & s_{1} & t_{1} \\
r_{2} & s_{2} & t_{2} \\
r_{3} & s_{3} & t_{3}
\end{array}\right) \quad \in \mathcal{S O}(3)
$$

Onde o vetor unitário $r=\left(r_{1}, r_{2}, r_{3}\right)^{t}$ denota a direção dos primeiros eixos do corpo rígido, $r_{1}$ é a componente na direção $e_{1}, r_{2}$ é a componente na direção $e_{2} e$ $r_{3}$ é a componente na direção $e_{3}$. Similarmente, o vetor unitário $s=\left(s_{1}, s_{2}, s_{3}\right)^{t} e$ $t=\left(t_{1}, t_{2}, t_{3}\right)^{t}$ darão a direção do segundo e terceiro eixos do corpo rígido. A evolução de $R$ pode agora ser expressa como:

$$
\dot{R(t)}=S(w(t)) R(t)
$$

Onde $w(t)$ é a velocidade angular da espaçonave em tempo $t$ (com relação aos eixos na espaçonave). $S(w)$ é uma matriz $3 \times 3$ antisimétrica definida por:

$$
S(w)=\left(\begin{array}{ccc}
0 & w_{3} & -w_{2} \\
-w_{3} & 0 & w_{1} \\
w_{2} & -w_{1} & 0
\end{array}\right)
$$

Forma Local:

A posição angular pode ser descrita localmente por 3 ângulos $\phi, \theta, \psi$, os quais representam consecutivamente no sentido horário as rotações ao redor dos eixos $r_{1}, r_{2}, r_{3}$ 
respectivamente. Tomando $r_{i}$ como o i-ésimo vetor da base em $\mathbb{R}^{3}$ obtemos a equação cinemática

$$
\begin{aligned}
\left(\begin{array}{l}
w_{1} \\
w_{2} \\
w_{3}
\end{array}\right)= & \left(\begin{array}{l}
\dot{\phi} \\
0 \\
0
\end{array}\right)+\left(\begin{array}{ccc}
1 & 0 & 0 \\
0 & \cos \phi & \sin \phi \\
0 & -\sin \phi & \cos \phi
\end{array}\right)\left(\begin{array}{c}
0 \\
\dot{\theta} \\
0
\end{array}\right) \\
& +\left(\begin{array}{ccc}
1 & 0 & 0 \\
0 & \cos \phi & \sin \phi \\
0 & -\sin \phi & \cos \phi
\end{array}\right)\left(\begin{array}{ccl}
\cos \theta & 0 & -\sin \theta \\
0 & 1 & 0 \\
\sin \theta & 0 & \cos \theta
\end{array}\right)\left(\begin{array}{c}
0 \\
0 \\
\dot{\psi}
\end{array}\right)
\end{aligned}
$$

Reunindo os términos e invertendo obtemos:

$$
\left(\begin{array}{c}
\dot{\phi} \\
\dot{\theta} \\
\dot{\psi}
\end{array}\right)=\left(\begin{array}{ccc}
1 & \sin \phi \tan \theta & \cos \phi \tan \theta \\
0 & \cos \phi & -\sin \phi \\
0 & \sin \phi \sec \theta & \cos \phi \sec \theta
\end{array}\right)\left(\begin{array}{c}
w_{1} \\
w_{2} \\
w_{3}
\end{array}\right)
$$

Mesmo que os ângulos não coincidem com a usual definição de ângulos de Euler, eles originam um conjunto conveniente de equações, ou seja uma carta de $\mathcal{S O}(3)$. De fato esta descrição é só valida localmente na região, $-\frac{\pi}{2}<\theta<\frac{\pi}{2}$, mas serve para mostrar que a equação $\dot{R}=S(w) R$, surge em uma variedade tridimensional a qual é o grupo de Lie $\mathcal{S O}(3)$, de matrizes reais ortogonais de $3 \times 3$ com determinante 1 .

2) Equação da Dinâmica:

A equação da Dinâmica: A aciona um propulsor.

Derivamos a equação Dinâmica por considerar um momento de balanço ao redor do centro de massa.

$$
J w=R h
$$

Onde $J$ é a matriz de inércia da astronave, $h$ o momento angular da astro-nave relativo aos eixos inerciais $e_{1}, e_{2}, e_{3}$ e $b_{i}$ são os eixos ao redor dos quais, o correspondente torque de controle de magnitude $\left\|b_{i}\right\| u_{i} i=1, \ldots, m$ é aplicado por médio a posição do par de propulsores, \|\| denota a norma euclideana em $\mathbb{R}^{3},\|a\|=\sqrt{\left|a^{t} a\right|}$, e $m$ designa o número de torques de controle. Obtemos a equação da Dinâmica que descreve o problema de controle de atitude.

$$
\begin{aligned}
\dot{R} & =S(w) R \quad R \in \mathcal{S O}(3) \\
J \dot{w} & =S(w) J w+\sum_{i=1}^{m} b_{i} u_{i} \quad w \in \mathbb{R}^{3}
\end{aligned}
$$


Onde $J$ é uma matriz $3 \times 3$, simétrica definida positiva, $J$ é chamada matriz inercial ou operador inercial, os autovetores de $J$ são chamados eixos principais, por simplicidade assumamos que os eixos $r, s, t$ são já os eixos principais portanto:

$$
J=\left(\begin{array}{ccl}
a_{1} & 0 & 0 \\
0 & a_{2} & 0 \\
0 & 0 & a_{3}
\end{array}\right) \quad a_{i}>0 \quad i=1,2,3
$$

Onde $b_{i}=\left(b_{i}^{1}, b_{i}^{2}, b_{i}^{3}\right)^{t} \in \mathbb{R}^{3} \quad i=1, \ldots, m$ e temos os seguintes campos vetoriais:

$$
\begin{aligned}
& f(R, w)=\left(\begin{array}{c}
S(w) R \\
J^{-1} S(w) J w
\end{array}\right) \\
& g_{i}(R, w)=\left(\begin{array}{c}
0 \\
J^{-1} b_{i}
\end{array}\right) \quad i=1, \ldots, m
\end{aligned}
$$

Vejamos uma condição necessária e suficiente para acessíbilidade Analizaremos 3 casos:

Caso I:

Para $m=3$ e $b_{1}, b_{2}, b_{3}$ são linearmente independentes. Temos que:

$$
\begin{aligned}
{\left[f, g_{i}\right] } & =\left[\left(\begin{array}{c}
S(w) R \\
J^{-1} S(w) J w
\end{array}\right),\left(\begin{array}{c}
0 \\
J^{-1} b_{i}
\end{array}\right)\right] \\
& =-\left(\begin{array}{c}
S\left(J^{-1} b_{i}\right) R \\
J^{-1} S\left(J^{-1} b_{i}\right) J w+J^{-1} S(w) b_{i}
\end{array}\right)
\end{aligned}
$$

Dado que $b_{1}, b_{2}, b_{3}$ são linearmente independentes, o vetor $S\left(J^{-1} b_{i}\right) R \quad i=1,2,3$ gera o espaço tangente $T_{R} S O(3)$ e, portanto, $\left\{g_{1}, g_{2}, g_{3},\left[f, g_{1}\right],\left[f, g_{2}\right],\left[f, g_{3}\right]\right\}$ geram $T_{(R, w)}\left(S O(3) \times \mathbb{R}^{3}\right)$ e concluímos que $C_{0}(R, w)=T_{(R, w)}\left(S O(3) \times \mathbb{R}^{3}\right)$ e pelo Teorema (1.3.2) o sistema é fortemente acessivel.

Caso II

Tomemos $m=2$ Calculemos:

$$
\begin{aligned}
{\left[f, \alpha_{1} g_{1}+\alpha_{2} g_{2}\right] } & =\left[\left(\begin{array}{c}
S(w) R \\
J^{-1} S(w) J w
\end{array}\right),\left(\begin{array}{c}
0 \\
\alpha_{1} J^{-1} b_{1}+\alpha_{2} J^{-1} b_{2}
\end{array}\right)\right] \\
& =-\left(\begin{array}{c}
S\left(\alpha_{1} J^{-1} b_{1}+\alpha_{2} J^{-1} b_{2}\right) R \\
J^{-1} S\left(\alpha_{1} J^{-1} b_{1}+\alpha_{2} J^{-1} b_{2}\right) J w+J^{-1} S(w)\left(\alpha_{1} b_{1}+\alpha_{2} b_{2}\right)
\end{array}\right)
\end{aligned}
$$




$$
\begin{aligned}
G & =\left[\left[f, \alpha_{1} g_{1}+\alpha_{2} g_{2}\right], \alpha_{1} g_{1}+\alpha_{2} g_{2}\right] \\
& =-\left[\left(\begin{array}{c}
S\left(\alpha_{1} J^{-1} b_{1}+\alpha_{2} J^{-1} b_{2}\right) R \\
J^{-1} S\left(\alpha_{1} J^{-1} b_{1}+\alpha_{2} J^{-1} b_{2}\right) J w \\
+J^{-1} S(w)\left(\alpha_{1} b_{1}+\alpha_{2} b_{2}\right)
\end{array}\right),\left(\begin{array}{c}
0 \\
\alpha_{1} J^{-1} b_{1}+\alpha_{2} J^{-1} b_{2}
\end{array}\right)\right] \\
& =\left(\begin{array}{c}
0 \\
2 J^{-1} S\left(\alpha_{1} J^{-1} b_{1}+\alpha_{2} J^{-1} b_{2}\right) J\left(\alpha_{1} J^{-1} b_{1}+\alpha_{2} J^{-1} b_{2}\right)
\end{array}\right)
\end{aligned}
$$

Por outro lado

$$
\begin{aligned}
{[f, G]=} & {\left[\left(\begin{array}{c}
S(w) R \\
J^{-1} S(w) J w
\end{array}\right),\left(\begin{array}{c}
0 \\
2 J^{-1} S\left(\alpha_{1} J^{-1} b_{1}+\alpha_{2} J^{-1} b_{2}\right) J\left(\alpha_{1} J^{-1} b_{1}+\alpha_{2} J^{-1} b_{2}\right)
\end{array}\right)\right] } \\
= & \left(\begin{array}{c}
S\left(2 J^{-1} S\left(\alpha_{1} J^{-1} b_{1}+\alpha_{2} J^{-1} b_{2}\right) J\left(\alpha_{1} J^{-1} b_{1}+\alpha_{2} J^{-1} b_{2}\right) R\right. \\
J^{-1} S\left(2 J^{-1} S\left(\alpha_{1} J^{-1} b_{1}+\alpha_{2} J^{-1} b_{2}\right) J\left(\alpha_{1} J^{-1} b_{1}+\alpha_{2} J^{-1} b_{2}\right)\right) J w \\
+J^{-1} S(w) J\left(2 J^{-1} S\left(\alpha_{1} J^{-1} b_{1}+\alpha_{2} J^{-1} b_{2}\right) J\left(\alpha_{1} J^{-1} b_{1}+\alpha_{2} J^{-1} b_{2}\right)\right)
\end{array}\right)
\end{aligned}
$$

Seja $B=<b_{1}, b_{2}>$ o subespaço de $\mathbb{R}^{3}$ gerado por $b_{1}, b_{2}$ e consideremos a condiçấo:

$$
\mathbb{R}^{3}=\operatorname{Span}\left\{b_{1}, b_{2}, S(w) J^{-1} w: w \in B=\operatorname{Span}\left\{b_{1}, b_{2}\right\}\right\}
$$

Esta condição é equivalente a:

$$
\mathbb{R}^{3}=\operatorname{Span}\left\{J^{-1} b_{1}, J^{-1} b_{2}, J^{-1} S(w) J^{-1} w: w \in B\right\} .
$$

Se a condição (5) é satisfeita, temos que $\left\{g_{1}, g_{2},\left\{G\left(\alpha_{1}, \alpha_{2}\right): \alpha_{1}, \alpha_{2} \in \mathbb{R}\right\},\left[f, g_{1}\right],\left[f, g_{2}\right]\right.$, $\left.\left\{\left[f, G\left(\alpha_{1}, \alpha_{2}\right)\right]: \alpha_{1}, \alpha_{2} \in \mathbb{R}\right\}\right\}$ geram $T_{x}\left(S O(3) \times \mathbb{R}^{3}\right)$ para todo $x=(R, w) \in$ $S O(3) \times \mathbb{R}^{3}$, e $C_{0}(x)=T_{x}\left(S O(3) \times \mathbb{R}^{3}\right)$ e, pelo Teorema (1.3.2) o sistema é fortemente acessivel, assim a condiçẫo (5) é uma condição suficiente para que o sistema seja fortemente acessivel.

Mostraremos agora, que a condição (5) é também necessária, tomemos a seguinte mudança de variável. $J w=\alpha_{1} b_{1}+\alpha_{2} b_{2}+\alpha_{3} S\left(b_{2}\right) b_{1}$ e substituindo na equação

$$
J \dot{w}=S(w) J w+b_{1} u_{1}+b_{2} u_{2} .
$$

obtemos o sistema:

$$
\begin{aligned}
& \dot{\alpha_{1}}=f_{1}\left(\alpha_{1}, \alpha_{2}, \alpha_{3}\right)+u_{1} \\
& \dot{\alpha_{2}}=f_{2}\left(\alpha_{1}, \alpha_{2}, \alpha_{3}\right)+u_{2} \\
& \dot{\alpha_{3}}=\alpha_{3} f_{3}\left(\alpha_{1}, \alpha_{2}\right)+\frac{f_{4}\left(\alpha_{1}, \alpha_{2}\right)}{\left\|S\left(b_{2}\right) b_{1}\right\|^{2}}
\end{aligned}
$$


E $f_{4}\left(\alpha_{1}, \alpha_{2}\right)=b_{1}^{t} S\left(b_{2}\right) S\left(\alpha_{1} b_{1}+\alpha_{2} b_{2}\right) J^{-1}\left(\alpha_{1} b_{1}+\alpha_{2}\right)$. Temos que $f_{4} \neq 0$ se, e só se, a condição (5) é satisfeita.

Agora se $f_{4}=0$, o sistema (6) o sistema não é acessível, dado que temos as seguintes variedades integrais maximais $\mathbb{R}^{3} \cap\left\{\alpha: \alpha_{3}=0\right\}, \mathbb{R}^{3} \cap\left\{\alpha: \alpha_{3}<0\right\}, \mathbb{R}^{3} \cap\{\alpha$ : $\left.\alpha_{3}>0\right\}$ o mais simplesmente, se o sistema (6) está em um estado onde $\alpha_{3}=0$, então $\alpha_{3}$ permanecerá zero, isto é, permanece constante. Assim, podemos concluir que se o sistema (6) é não-acessível então o sistema (4) não é acessivel. Portanto concluímos que a condição (5) é necessária e suficiente para a acessibilidade.

Caso III

Quando $m=1$, consideremos os seguintes campos vetoriais $g_{1} e$

$$
\left[f, g_{1}\right]=\left(\begin{array}{c}
S\left(J^{-1} b_{1}\right) R \\
J^{-1} S\left(J^{-1} b_{1}\right) J w+J^{-1} S(w) b_{1}
\end{array}\right)
$$

E o campo vetorial

$$
\begin{aligned}
G_{2}= & {\left[\left[f, g_{1}\right], g_{1}\right]=\left[-\left(\begin{array}{c}
S\left(J^{-1} b_{1}\right) R \\
J^{-1} S\left(J^{-1} b_{1}\right) J w+J^{-1} S(w) b_{1}
\end{array}\right),\left(\begin{array}{c}
0 \\
J^{-1} b_{1}
\end{array}\right)\right] } \\
= & \left(\begin{array}{c}
0 \\
2 J^{-1} S\left(J^{-1} b_{1}\right) b_{1}
\end{array}\right) \\
{\left[f, \alpha_{1} g_{1}+\alpha_{2} G_{2}\right]=} & {\left.\left[\begin{array}{c}
S(w) R \\
J^{-1} S(w) J w
\end{array}\right),\left(\begin{array}{c}
0 \\
\alpha_{1} J^{-1} b_{1}+2 \alpha_{2} J^{-1} S\left(J^{-1} b_{1}\right) b_{1}
\end{array}\right)\right] } \\
= & -\left(\begin{array}{c}
S\left(\alpha_{1} J^{-1} b_{1}+2 \alpha_{2} J^{-1} S\left(J^{-1} b_{1}\right) b_{1}\right) R \\
J^{-1} S\left(\alpha_{1} J^{-1} b_{1}+2 \alpha_{2} J^{-1} S\left(J^{-1} b_{1}\right) b_{1}\right) J w \\
+J^{-1} S(w) J\left(\alpha_{1} J^{-1} b_{1}+2 \alpha_{2} J^{-1} S\left(J^{-1} b_{1}\right) b_{1}\right)
\end{array}\right) .
\end{aligned}
$$

Calculemos também

$$
\begin{aligned}
G_{3}\left(\alpha_{1}, \alpha_{2}\right) & =\left[\left[f,\left(\alpha_{1} g_{1}+\alpha_{2} G_{2}\right)\right],\left(\alpha_{1} g_{1}+\alpha_{2} G_{2}\right]\right. \\
& =-\left(\begin{array}{c}
0 \\
J^{-1} S\left(\alpha_{1} J^{-1} b_{1}+2 \alpha_{2} J^{-1} S\left(J^{-1} b_{1}\right) b_{1}\right)\left(\alpha_{1} b_{1}+2 \alpha_{2} S\left(J^{-1} b_{1}\right) b_{1}\right) \\
+J^{-1} S\left(\alpha_{1} J^{-1} b_{1}+2 \alpha_{2} J^{-1} S\left(J^{-1} b_{1}\right) b_{1}\right)\left(\alpha_{1} b_{1}+2 \alpha_{2} S\left(J^{-1} b_{1}\right) b_{1}\right)
\end{array}\right)
\end{aligned}
$$


e finalmente

$\left[f, G_{3}\right]=\left(\begin{array}{c}S\left(2 J^{-1} S\left(\alpha_{1} J^{-1} b_{1}+2 \alpha_{2} J^{-1} S\left(J^{-1} b_{1}\right) b_{1}\left(\alpha_{1} b_{1}+2 \alpha_{2} S\left(J^{-1} b_{1}\right) b_{1}\right)\right) R\right. \\ J^{-1} S\left(2 J^{-1} S\left(\alpha_{1} J^{-1} b_{1}+2 \alpha_{2} J^{-1} S\left(J^{-1} b_{1}\right) b_{1}\right)\left(\alpha_{1} b_{1}+2 \alpha_{2} S\left(J^{-1} b_{1}\right) b_{1}\right)\right. \\ +J^{-1} S(w) J\left(2 J^{-1} S\left(\alpha_{1} J^{-1} b_{1}+2 \alpha_{2} J^{-1} S\left(J^{-1} b_{1}\right) b_{1}\right)\left(\alpha_{1} b_{1}+2 \alpha_{2} S\left(J^{-1} b_{1}\right) b_{1}\right)\right.\end{array}\right)$

Se Consideramos a condiçâo

$$
\mathbb{R}^{3}=\operatorname{Span}\left\{b_{1}, S\left(b_{1}\right) J^{-1} b_{1}, S(w) J^{-1} w: w \in \operatorname{Span}\left\{b_{1}, S\left(b_{1}\right) J^{-1} b_{1}\right\}\right\}
$$

A qual é equivalente a:

$$
\mathbb{R}^{3}=\operatorname{Span}\left\{\begin{array}{l}
J^{-1} b_{1}, 2 J^{-1} S\left(J^{-1} b_{1}\right) b_{1}, 2 J^{-1} S\left(\alpha_{1} J^{-1} b_{1}+2 \alpha_{2} J^{-1} S\left(J^{-1} b_{1}\right) b_{1}\right) \\
\\
\left.J\left(\alpha_{1} J^{-1} b_{1}+2 \alpha_{2} J^{-1} S\left(J^{-1} b_{1}\right) b_{1}\right): \quad \alpha_{1}, \alpha_{2} \in \mathbb{R}^{3}\right\}
\end{array}\right.
$$

Se a condição (7) é satisfeita, temos que $\left\{g_{1}, g_{2},\left\{G_{3}\left(\alpha_{1}, \alpha_{2}\right): \alpha_{1}, \alpha_{2} \in\right.\right.$ $\left.\left.\mathbb{R}^{3}\right\},\left[f, g_{1}\right],\left[f, G_{2}\right],\left\{\left[f, G_{3}\left(\alpha_{1}, \alpha_{2}\right)\right]: \alpha_{1}, \alpha_{2} \in \mathbb{R}\right\}\right\}$ geram $T_{x}\left(S O(3) \times \mathbb{R}^{3}\right)$ para todo $x=(R, w) \in S O(3) \times \mathbb{R}^{3}$, e por o Teorema (1.3.2) o sistema é fortemente acessível, assim a condição (7) é suficiente para a acessibilidade. Agora vejamos que também, é necessária. Consideremos a seguinte substituição de variáveis:

$$
J w=\alpha_{1} b_{1}+\alpha_{2} S\left(b_{1}\right) J^{-1} b_{1}+\alpha_{3} S\left(b_{1}\right) S\left(b_{1}\right) J^{-1} b_{1},
$$

na equação :

$$
J \dot{w}=S(w) J w+b_{1} u_{1}
$$

Obtenendo assim o sistema

$$
\begin{aligned}
& \dot{\alpha_{1}}=f_{1}\left(\alpha_{1}, \alpha_{2}, \alpha_{3}\right)+u_{1} \\
& \dot{\alpha_{2}}=f_{2}\left(\alpha_{1}, \alpha_{2}, \alpha_{3}\right) \\
& \dot{\alpha_{3}}=\alpha_{3} f_{3}\left(\alpha_{1}, \alpha_{2}\right)+\frac{f_{4}\left(\alpha_{1}, \alpha_{2}\right)}{\left\|S\left(b_{1}\right) S\left(b_{1}\right) J^{-1} b_{1}\right\|^{2}}
\end{aligned}
$$

Onde

$$
f_{4}\left(\alpha_{1}, \alpha_{2}\right)=-b_{1}^{t} J^{-1} S\left(b_{1}\right) S\left(b_{1}\right) S\left(\alpha_{1} b_{1}+\alpha_{2} S\left(b_{1}\right) J^{-1} b_{1}\right) J^{-1}\left(\alpha_{1} b_{1}+\alpha_{2} S\left(b_{1}\right) J^{-1} b_{1}\right)
$$

Temos que $f_{4} \neq 0$ se, e só se, é satisfeita a condição (7), se $f_{4}=0$, o sistema (8) não pode ser acessível, dado que se $\alpha_{3}=0$, permanece constante o qual implica o mesmo para o sistema (4). Assim a condição (7) é necessária e suficiente para a acessibilidade. 


\section{Capítulo 2}

\section{Geometria Simplética}

\subsection{Introdução}

Neste capítulo, apresentamos alguns resultados sobre variedades de Poisson e variedades simpléticas, que utilizaremos no estudo de um sistema Hamiltoniano afim de controle. Definiremos uma lei de composição chamada o colchete de Lie no espaço das funções diferenciáveis definidas numa variedade, que torna este espaço numa álgebra de Lie; pensaremos no colchete de Poisson como um elemento essencial de uma estrutura simplética. A variedade de Poisson aparece como uma generalização da variedade simplética, para maiores detalhes veja [1], [2], [14].

\subsection{Variedade de Poisson}

Definição 2.2.1 Seja $\mathcal{M}$ uma variedade. Uma estrutura de Poisson em $\mathcal{M}$ é determinada por uma transformaçâo bilinear $\{\cdot, \cdot\}: C^{\infty}(\mathcal{M}) \times C^{\infty}(\mathcal{M}) \longrightarrow C^{\infty}(\mathcal{M})$, chamada o colchete de Poisson, satisfazendo as seguintes propiedades:

i) Anti-simétrica:

$$
\{F, G\}=-\{G, F\}
$$

ii) Identidade de Jacobi:

$$
\{F,\{G, H\}\}+\{G,\{H, F\}\}+\{H,\{F, G\}\}=0
$$

iii) Regra de Leibnitz:

$$
\{F, G H\}=\{F, G\} H+G\{F, H\}
$$


onde $F, G$ e $H \in C^{\infty}(\mathcal{M})$.

Observação 2.2.1 A variedade $\mathcal{M}$ com a estrutura de Poisson é chamada de variedade de Poisson.

Observação 2.2.2 $O$ conjunto $C^{\infty}(\mathcal{M})$ com o colchete de Poisson é uma Álgebra de Lie.

Observação 2.2.3 A operação colchete $\{\cdot, \cdot\}$ é uma derivação em cada argumento.

Para cada $F \in C^{\infty}(\mathcal{M})$ e arbitrário $x \in \mathcal{M}$ definamos a transformação $\mathcal{X}_{F}(x)$ : $C^{\infty}(\mathcal{M}) \longrightarrow \mathbb{R}$

$$
\mathcal{X}_{F}(x) G=\{F, G\}(x)
$$

Pela regra de Leibnitz temos

$$
\begin{aligned}
\mathcal{X}_{F}(x)(G H) & =\{F, G H\}(x)=\{F, G\}(x) H(x)+G(x)\{F, H\}(x) \\
& =\mathcal{X}_{F} G(x) H(x)+G(x) \mathcal{X}_{F} H(x)
\end{aligned}
$$

Logo, concluímos que $\mathcal{X}_{F}(x) \in T_{x} \mathcal{M}$ para qualquer $x \in \mathcal{M}$. Assim para cada $F \in C^{\infty}(\mathcal{M})$ existe um campo vetorial $\mathcal{X}_{F}$ em $\mathcal{M}$ tal que

$$
\mathcal{X}_{F}(G)=\{F, G\}
$$

$\mathcal{X}_{F}$ é chamado campo vetorial Hamiltoniano com função de energia ou função Hamiltoniana $F$. Recordemos que $V^{\infty}(M)$ denota o espaço vetorial dos campos vetoriais suaves definidos em $\mathcal{M}$, e que também é uma álgebra de Lie. A transformação $F \longrightarrow \mathcal{X}_{F}$ é um morfismo de álgebras de Lie, que faz a passagem de $C^{\infty}(\mathcal{M})$ com o colchete de Poisson para $V(\mathcal{M})$ com o colchete de Lie usual.

Vejamos isso mediante a seguinte proposição.

Proposição 2.2.1 Sejam $F, G \in C^{\infty}(\mathcal{M})$. Temos que

$$
\mathcal{X}_{\{F, G\}}=\left[\mathcal{X}_{F}, \mathcal{X}_{G}\right]
$$

Demonstração: Seja $H \in C^{\infty}(\mathcal{M})$, segue da definição de campo vetorial Hamiltoniano e da identidade de Jacobi que:

$$
\begin{aligned}
\mathcal{X}_{\{F, G\}}(H) & =\{\{F, G\}, H\} \\
& =\{F,\{G, H\}\}-\{G,\{F, H\}\} \\
& =\mathcal{X}_{F}\left(\mathcal{X}_{G}(H)\right)-\mathcal{X}_{G}\left(\mathcal{X}_{F}(H)\right) \\
& =\left[\mathcal{X}_{F}, \mathcal{X}_{G}\right](H)
\end{aligned}
$$


Assumamos que $F$ é fixo

$$
\{F, G\}(x)=\mathcal{X}_{F}(x)(G)(x)=d G(x)\left(\mathcal{X}_{F}\right)(x)
$$

Portanto quando $G$ varia $\{F, G\}(x)$ só depende de $d G(x)$, similarmente quando $G$ é fixo

$$
\{F, G\}(x)=-\{G, F\}=-\mathcal{X}_{G}(F)(x)=-d F\left(\mathcal{X}_{G}\right)(x)
$$

Ou seja quando $F$ varia $\{F, G\}$ só depende de $d F(x)$, temos que a transformação $F \longrightarrow d F(x)$, que vai de $C^{\infty}(\mathcal{M})$ em $T_{x}^{*} \mathcal{M}$, é sobrejetora e como a transformação $(F, G) \longrightarrow\{F, G\}$ é bilinear e antisimetrica, portanto para todo $x \in \mathcal{M}$ existe uma forma bilinear e antisimétrica $W(x)$ no espaço tangente $T^{*} \mathcal{M}$, tal que para todo $F, G \in C^{\infty}(\mathcal{M})$

$$
\{F, G\}(x)=W(x)(d F(x), d G(x))
$$

onde $W$ é chamado o tensor de Poisson. Em coordenadas locais $x_{1}, \ldots, x_{r}$ uma estrutura de Poisson é determinada pelas funções localmente suaves $w_{i j} \quad 1 \leq i \leq r, 1 \leq j \leq r$, tal que o colchete de Poisson é dado por:

$$
\{F, G\}(x)=\sum_{i, j=1}^{r} w_{i j}(x) \frac{\partial F(x)}{\partial x_{i}} \frac{\partial G(x)}{\partial x_{j}}
$$

Onde $w_{i j}$ é determinada por

$$
w_{i j}(x)=\left\{x_{i}, x_{j}\right\} \quad 1 \leq i \leq r, \quad 1 \leq j \leq r .
$$

$\mathrm{Da}$ antisimetria e a identidade de Jacobi temos que as funções $w_{i, j}$ devem satisfazer as seguintes relações:

$$
\begin{gathered}
w_{i j}=-w_{j i}(*) \\
\sum_{l=1}^{r}\left(w_{l j} \frac{\partial w_{i k}}{\partial x_{l}}+w_{l i} \frac{\partial w_{k j}}{\partial x_{l}}+w_{l k} \frac{\partial w_{j l}}{\partial x_{l}}\right)=0 .
\end{gathered}
$$

Localmente qualquer colchete de Poisson é determinado pela matriz antisimétrica

$$
\begin{aligned}
W(x)=\left(w_{i j}(x)\right) & \\
i & =1, \ldots, r \\
j & =1, \ldots, r
\end{aligned}
$$

Onde $w_{i j}(x)$ satisfaz $(*)$ e $(* *)$, se temos $w_{i j}(x)$ tal que satisfazem $(*)$ e $(* *)$ elas definem um colchete de Poisson onde, $W(x)$ é chamada matriz estrutura. O posto do colchete de Poisson em $x \in \mathcal{M}$ é definido como o posto de $W(x)$. Dado que $W(x)$ é anti-simétrica o posto é par. Dizemos que o colchete de Poisson é não-degenerado se o posto $W(x)=\operatorname{dim} \mathcal{M}$ para todo $x \in \mathcal{M}$. 
Um automorfismo de Poisson é definido como um difeomorfismo $J: \mathcal{M} \longrightarrow \mathcal{M}$, onde $\mathcal{M}$ é uma variedade de Poisson tal que

$$
\{F o J, G o J\}=\{F, G\} o J \quad \text { para todo } F, G \in C^{\infty}(\mathcal{M})
$$

Dizemos que um campo vetorial $\mathcal{X}$ em $\mathcal{M}$ é um automorfismo de Poisson infinitesimal se o fluxo ou grupo a um parâmetro $\mathcal{X}^{t}$ de $\mathcal{X}$ são automorfismos de Poisson para todo $t$ para o qual $\mathcal{X}^{t}$ esta definida, ou seja

$$
\left\{F \circ \mathcal{X}^{t}, G \circ \mathcal{X}^{t}\right\}=\{F, G\} \circ \mathcal{X}^{t}
$$

Proposição 2.2.2 Seja $\mathcal{M}$ uma variedade de Poisson. Um campo vetorial $\mathcal{X}$ definido em $\mathcal{M}$ é um automorfismo de Poisson infinitesimal se e só se

$$
\mathcal{X}(\{F, G\})=\{\mathcal{X}(F), G\}+\{F, \mathcal{X}(G)\} \text { para todo } F, G \in C^{\infty}(\mathcal{M})
$$

Demonstração: Nossa demonstração esta baseada na seguinte igualdade

$$
\begin{aligned}
& \frac{d}{d t}\left\{F \circ \mathcal{X}^{t}, G \circ \mathcal{X}^{t}\right\} \circ \mathcal{X}^{-t} \backslash_{t=t_{0}}=\lim _{h \rightarrow 0} \frac{\left\{F \circ \mathcal{X}^{t+h}, G \circ \mathcal{X}^{t+h}\right\} \circ \mathcal{X}^{-t-h}-\left\{F \circ \mathcal{X}^{t}, G \circ \mathcal{X}^{t}\right\} \circ \mathcal{X}^{-t}}{h} \\
& =-\lim _{h \rightarrow 0} \frac{\left\{F \circ \mathcal{X}^{t+h}, G \circ \mathcal{X}^{t+h}\right\} \circ \mathcal{X}^{-t}\left(\mathcal{X}^{-h}\right)}{-h} \\
& \frac{-\left\{F \circ \mathcal{X}^{t+h}, G \circ \mathcal{X}^{t+h}\right\} \circ \mathcal{X}^{-t}}{-h}+\lim _{h \rightarrow 0} \frac{\left\{F \circ \mathcal{X}^{t+h}, G \circ \mathcal{X}^{t+h}\right\} \circ \mathcal{X}^{-t}}{h} \\
& \underline{-\left\{F \circ \mathcal{X}^{t}, G \circ \mathcal{X}^{t}\right\} \circ \mathcal{X}^{-t}} \\
& =-\left(\mathcal{X}\left(\left\{F \circ \mathcal{X}^{t}, G \circ \mathcal{X}^{t}\right\}\right)\right) \circ \mathcal{X}^{-t} \\
& +\lim _{h \rightarrow 0} \frac{\left\{F \circ \mathcal{X}^{t}\left(\mathcal{X}^{h}\right)-F \circ \mathcal{X}^{t}, G \circ \mathcal{X}^{t+h}\right\} \circ \mathcal{X}^{-t}}{\left.\mathcal{X}^{t+h} \mathcal{X}^{-t} \mathcal{X}^{t} \mathcal{X}^{t}\right\} \mathcal{X}^{-t}} \\
& +\left\{F \circ \mathcal{X}^{t}, G \circ \mathcal{X}^{t+h}\right\} \circ \mathcal{X}^{-t}-\left\{F \circ \mathcal{X}^{t}, G \circ \mathcal{X}^{t}\right\} \circ \mathcal{X}^{-t} \\
& =-\left(\mathcal{X}\left(\left\{F \circ \mathcal{X}^{t}, G \circ \mathcal{X}^{t}\right\}\right)\right) \circ \mathcal{X}^{-t} \\
& +\left\{\mathcal{X}\left(F \circ X^{t}\right), G \circ \mathcal{X}^{t}\right\} \circ \mathcal{X}^{-t} \\
& +\lim _{h \rightarrow 0} \frac{\left\{F \circ \mathcal{X}^{t}, G \circ \mathcal{X}^{t}\left(\mathcal{X}^{h}\right)-G \circ \mathcal{X}^{t}\right\} \circ \mathcal{X}^{-t}}{h} \\
& =-\left(\mathcal{X}\left(\left\{F \circ \mathcal{X}^{t}, G \circ \mathcal{X}^{t}\right\}\right)\right) \circ \mathcal{X}^{-t} \\
& +\left\{\mathcal{X}\left(F \circ \mathcal{X}^{t}\right), G \circ X^{t}\right\} \circ X^{-t} \\
& +\left\{F \circ \mathcal{X}^{t}, \mathcal{X}\left(G \circ \mathcal{X}^{t}\right)\right\} \circ \mathcal{X}^{-t}
\end{aligned}
$$

Portanto temos que:

$$
\begin{aligned}
& \frac{d}{d t}\left\{f \circ \mathcal{X}^{t}, g \circ \mathcal{X}^{t}\right\} \circ \mathcal{X}^{-t} \backslash_{t=t_{0}}=-\left(\mathcal{X}\left(\left\{F \circ \mathcal{X}^{t_{0}}, G \circ \mathcal{X}^{t_{0}}\right\}\right)\right) \circ \mathcal{X}^{-t_{0}}+\left\{\mathcal{X}\left(F \circ \mathcal{X}^{t_{0}}\right), G \circ\right. \\
& \left.\mathcal{X}^{t_{0}}\right\} \circ \mathcal{X}^{-t_{0}}+\left\{F \circ \mathcal{X}^{t_{0}}, \mathcal{X}\left(G \circ \mathcal{X}^{t_{0}}\right)\right\} \circ \mathcal{X}^{-t_{0}}
\end{aligned}
$$


Se $X$ é um automorfismo de Poisson, ou seja $\left\{F \circ \mathcal{X}^{t}, G \circ \mathcal{X}^{t}\right\} \circ \mathcal{X}^{-t}=\{F, G\}$, a expressão da direita não depende de $t$, se anula, e tomando $t_{0}=0$, temos que

$$
\mathcal{X}(\{F, G\})=\{\mathcal{X}(F), G\}+\{F, \mathcal{X}(G)\}
$$

Agora se $\mathcal{X}(\{F, G\})=\{\mathcal{X}(F), G\}+\{F, \mathcal{X}(G)\}$, portanto

$$
\frac{d}{d t}\left\{F \circ \mathcal{X}^{t}, G \circ \mathcal{X}^{t}\right\} \circ \mathcal{X}^{-t}=0 \quad \forall t
$$

isto é

$$
\left\{F \circ \mathcal{X}^{t}, G \circ \mathcal{X}^{t}\right\} \circ \mathcal{X}^{-t}=\{F, G\} \quad \forall t
$$

Aplicando $\mathcal{X}^{t}$ a ambos lados

$$
\left\{F \circ \mathcal{X}^{t}, G \circ \mathcal{X}^{t}\right\}=\{F, G\} \circ \mathcal{X}^{t}
$$

isto completa nossa demonstração.

Da Proposição (2.2.2) e da identidade de Jacobi temos que qualquer campo vetorial Hamiltoniano é um automorfismo de Poisson infinitesimal.

Si $\mathcal{M}$ for de dimensão par, diremos que $(q, p)=\left(q_{1}, \ldots, q_{n}, p_{1}, \ldots, p_{n}\right)$ são coordenadas canônicas se e só se $\left\{p_{i}, q_{j}\right\}=\delta_{i j},\left\{q_{i}, q_{j}\right\}=0,\left\{p_{i}, p_{j}\right\}=0 \quad 1 \leq i \leq n, 1 \leq j \leq n$.

Teorema 2.2.1 Seja $\mathcal{M}$ uma variedade tal que $\operatorname{dim} \mathcal{M}=2 n$, com colchete de Poisson não-degenerado então localmente numa vizinhança $x_{0} \in \mathcal{M}$, podemos encontrar coordenadas canônicas $(q, p)=\left(q_{1}, \ldots, q_{n}, p_{1}, \ldots, p_{n}\right)$ tal que

$$
\{F, G\}(q, p)=\sum_{i=1}^{n}\left(\frac{\partial F}{\partial p_{i}} \frac{\partial G}{\partial q_{i}}-\frac{\partial F}{\partial q_{i}} \frac{\partial G}{\partial p_{i}}\right)(q, p)
$$

Demonstração: Veja a prova en [16] pagina 359.

Para qualquer colchete de Poisson o campo vetorial Hamiltoniano é um automorfismo de Poisson, a recíproca so é verdade localmente se o colchete é não-degenerado, vejamos isso em a seguinte proposição

Proposição 2.2.3 Seja $\mathcal{M}$ uma variedade de Poisson com colchete de Poisson nãodegenerado. Se o campo vetorial $\mathcal{X}$ em $\mathcal{M}$ é um automorfismo de Poisson infinitesimal então localmente em torno de $x_{0} \in \mathcal{M}$ existe $H \in C^{\infty}(\mathcal{M})$ tal que $\mathcal{X}=\mathcal{X}_{H}$ 
Demonstração: Pelo Teorema (2.2.1) podemos encontrar coordenadas canônicas $(q, p)=\left(q_{1}, \ldots, q_{n}, p_{1}, \ldots, p_{n}\right)$ ao redor de $x_{0} \in \mathcal{M}$. O campo vetorial $\mathcal{X}$ nas coordenadas canônicas é dado por $\mathcal{X}=\left(\mathcal{X}_{1}^{q}, \ldots, \mathcal{X}_{n}^{q}, \mathcal{X}_{1}^{p}, \ldots, \mathcal{X}_{n}^{p}\right)^{t}$. Dado que $\mathcal{X}$ é um automorfismo de Poisson infinitesimal temos

$$
\begin{aligned}
0 & =\mathcal{X}\left(\delta_{i j}\right)=\mathcal{X}\left(\left\{p_{i}, q_{j}\right\}\right)=\left\{\mathcal{X}\left(p_{i}\right), q_{j}\right\}+\left\{p_{i}, \mathcal{X}\left(q_{j}\right)\right\} \\
& =\left\{\mathcal{X}_{i}^{p}, q_{j}\right\}+\left\{p_{i}, \mathcal{X}_{j}^{q}\right\}=\frac{\partial \mathcal{X}_{i}^{p}}{\partial p_{j}}+\frac{\partial \mathcal{X}_{j}^{q}}{\partial q_{i}}
\end{aligned}
$$

Por outro lado

$$
\begin{aligned}
& 0=\mathcal{X}\left(\left\{q_{i}, q_{j}\right\}\right)=\left\{\mathcal{X}_{i}^{q}, q_{j}\right\}+\left\{q_{i}, \mathcal{X}_{j}^{q}\right\}=\frac{\partial \mathcal{X}_{i}^{q}}{\partial p_{j}}-\frac{\partial \mathcal{X}_{j}^{q}}{\partial p_{i}} \\
& 0=\mathcal{X}\left(\left\{p_{i}, p_{j}\right\}\right)=\left\{\mathcal{X}_{i}^{p}, p_{j}\right\}+\left\{p_{i}, \mathcal{X}_{j}^{p}\right\}=-\frac{\partial \mathcal{X}_{i}^{p}}{\partial q_{j}}+\frac{\partial \mathcal{X}_{j}^{p}}{\partial q_{i}} .
\end{aligned}
$$

O vetor linha $\left(-\mathcal{X}_{1}^{p}, \ldots,-\mathcal{X}_{n}^{p}, \mathcal{X}_{1}^{q}, \ldots, \mathcal{X}_{n}^{q}\right)$ representa uma 1 -forma fechada. De fato, seja

$$
\alpha=\sum_{i=1}^{n}\left(-\mathcal{X}_{i}^{p} d q_{i}+\mathcal{X}_{i}^{q} d p_{i}\right)
$$

logo, temos que

$$
d \alpha=\sum_{i<j}\left(-\frac{\partial \mathcal{X}_{i}^{p}}{\partial q_{j}}+\frac{\partial \mathcal{X}_{j}^{p}}{\partial q_{i}}\right) d q_{j} \wedge d q_{i}-\sum_{i, j}\left(\frac{\partial \mathcal{X}_{i}^{p}}{\partial p_{j}}+\frac{\partial \mathcal{X}_{j}^{q}}{\partial q_{i}}\right) d p_{j} \wedge d q_{i}+\sum_{i<j}\left(\frac{\partial \mathcal{X}_{i}^{q}}{\partial p_{j}}-\frac{\partial \mathcal{X}_{j}^{q}}{\partial p_{i}}\right) d p_{j} \wedge d p_{i} .
$$

Do anterior temos

$$
\begin{array}{r}
\frac{\partial \mathcal{X}_{i}^{p}}{\partial p_{j}}+\frac{\partial \mathcal{X}_{j}^{q}}{\partial q_{i}}=0 \\
\frac{\partial \mathcal{X}_{i}^{q}}{\partial p_{j}}-\frac{\partial \mathcal{X}_{j}^{q}}{\partial p_{i}}=0 \\
-\frac{\partial \mathcal{X}_{i}^{p}}{\partial q_{j}}+\frac{\partial \mathcal{X}_{j}^{p}}{\partial q_{i}}=0
\end{array}
$$

Portanto $d \alpha=0$, ou seja $\alpha$ é fechada pelo o lema de Poincaré temos que existe $H: \mathcal{M} \longrightarrow \mathbb{R}, H(q, p)$ tal que:

$$
-\mathcal{X}_{i}^{p}=\frac{\partial H}{\partial q_{i}} \quad \mathcal{X}_{i}^{q}=\frac{\partial H}{\partial p_{i}} \quad 1 \leq i \leq n
$$

o qual demonstra a Proposição. 


\subsection{Variedade Simplética}

Seja $\{\cdot, \cdot\}$ um colchete de Poisson não-degenerado em $\mathcal{M}$, localmente é dado pela matriz estrutura anti-simétrica $W(x)$. Definamos a transformação bilinear

$$
w_{x}: T_{x} \mathcal{M} \times T_{x} \mathcal{M} \longrightarrow \mathbb{R}
$$

Dada por

$$
w_{x}\left(\mathcal{X}_{F}(x), \mathcal{X}_{G}(x)\right)=\{F, G\}(x)
$$

A transformação bilinear $w_{x}$ é definida, em princípio, para vetores tangentes da forma $\mathcal{X}_{F}(x) \in T_{x} \mathcal{M}$, generalizemos nossa definição. Podemos escolher $2 n$ funções $F_{1}, \ldots, F_{2 n} \in C^{\infty}(\mathcal{M})$ tal que $\mathcal{X}_{F_{1}}(x), \ldots, \mathcal{X}_{F_{2 n}}(x)$ são independentes e constituem uma base de $T_{x} \mathcal{M}$. Sejam $G_{1}, G_{2} \in T_{x} \mathcal{M}$ quaisquer, eles são uma combinação linear dos campos vetoriais $\mathcal{X}_{F_{1}}(x), \ldots, \mathcal{X}_{F_{2 n}}(x)$, ou seja,

$$
G_{1}=\sum_{i=1}^{2 n} \alpha_{i} \mathcal{X}_{F_{i}}(x) \quad G_{2}=\sum_{j=1}^{2 n} \beta_{j} \mathcal{X}_{F_{j}}(x)
$$

Pela linearidade da aplicação $w_{x}$, temos

$$
\begin{aligned}
w_{x}\left(G_{1}, G_{2}\right) & =w_{x}\left(\sum_{i=1}^{2 n} \alpha_{i} \mathcal{X}_{F_{i}}(x), \sum_{j=1}^{2 n} \beta_{j} \mathcal{X}_{F_{j}}(x)\right) \\
& =\sum_{i=1}^{2 n} \alpha_{i}\left(\sum_{j=1}^{2 n} \beta_{j} w_{x}\left(\mathcal{X}_{F_{i}}(x), \mathcal{X}_{F_{j}}(x)\right)\right) \\
& =\sum_{i=1}^{2 n} \alpha_{i}\left(\sum_{j=1}^{2 n} \beta_{j}\left\{F_{i}, F_{j}\right\}(x)\right) .
\end{aligned}
$$

Portanto $w_{x}$ está bem definida como uma transformação bilinear.

Em coordenadas locais o vetor coluna $\mathcal{X}_{H}(x)$ é dado por $\left(d H(x) w_{x}\right)^{t}, d H(x)$ é um vetor linha, por outro lado, temos que $w_{x}$ tem a matriz de representação:

$$
\left[w_{x}\right]=-(W(x))^{-1}
$$

Assim, temos uma 2-forma diferenciável, que será denominada de uma forma simplética em $\mathcal{M}$. Em coordenadas canônicas $(q, p)$ podemos escrever

$$
w_{x}=W(x)=\left(\begin{array}{cc}
0 & -I_{n} \\
I_{n} & 0
\end{array}\right) .
$$


Dado que $\{F, G\}=-\{G, F\}=-\mathcal{X}_{G}(F)=-d F\left(\mathcal{X}_{G}\right)$, o campo vetorial Hamiltoniano $\mathcal{X}_{H}$ é determinado pela relação

$$
w_{x}\left(\mathcal{X}_{H}(x), \mathcal{Y}\right)=-d H(x)(\mathcal{Y}) \text { para qualquer } \mathcal{Y} \in T_{x} \mathcal{M}, \quad x \in \mathcal{M}
$$

Formalizemos o que entenderemos por uma estrutura simplética.

Definição 2.3.1 Seja $\mathcal{M}$ uma variedade diferenciável. Uma estrutura simplética em $\mathcal{M}$ é uma 2-forma $w$ que satisfaz:

i) $w$ é não-degenerada, ou seja, para todo $0 \neq \mathcal{X} \in T_{x} \mathcal{M}$ existe $\mathcal{Y} \in T_{x} \mathcal{M}$ tal que $w_{x}(\mathcal{X}, \mathcal{Y}) \neq 0$.

ii) w é uma 2-forma fechada isto é $d w=0$.

De (i) segue que $\mathcal{M}$ é de dimensão par. De (i) e (ii) pode ser deduzido que existem coordenadas locais $p_{1}, \ldots, p_{n}, q_{1}, \ldots, q_{n}$ para $\mathcal{M}$ tal que:

$$
w=\sum_{i=1}^{n} d p_{i} \wedge d q_{i}
$$

Isto é mais conhecido como o Teorema de Darboux's, para a demonstração do teorema veja [2] pagina 230 .

Definição 2.3.2 Seja $\mathcal{M}$ uma variedade diferenciável e $\omega$ uma estrutura simplética definida em $\mathcal{M},(\mathcal{M}, w)$ é chamada uma variedade simplética.

Daremos agora algumas propiedades que usaremos posteriormente

Propriedade 1: Seja $\mathcal{Q}$ uma variedade de dimensão $n$, o fibrado cotangente $T^{*} \mathcal{Q}=$ $\mathcal{M}$, tem uma estrutura simplética canônica.

Prova: : Podemos definir uma 1-forma $\Theta$ em $T^{*} \mathcal{Q}$ como segue. Seja $\alpha \in$ $T^{*} \mathcal{Q}, \quad \mathcal{X} \in T_{\alpha}\left(T^{*} \mathcal{Q}\right)$ um vetor tangente ao fibrado cotangente no ponto $\alpha \in T^{*} \mathcal{Q}$. $\pi$ denota a projeção de $T^{*} \mathcal{Q}$ em $\mathcal{Q}\left(\pi: T^{*} \mathcal{Q} \longrightarrow \mathcal{Q}\right)$ e $\pi_{*}$ a derivada de $\pi, \pi_{*}$ : $T\left(T^{*} \mathcal{Q}\right) \longrightarrow T \mathcal{Q}$. Definamos a 1 -forma $\Theta$ em $T^{*} \mathcal{Q}$ pela relação:

$$
\Theta_{\alpha}(\mathcal{X})=\alpha\left(\pi_{*}(\mathcal{X})\right)
$$

Em coordenadas locais temos que

$$
\Theta=\sum_{i=1}^{n} p_{i} d q_{i},
$$


onde $q_{i}$ são coordenadas locais para $\mathcal{Q}$ e $p_{i}$ são coordenadas do fibrado $T^{*} \mathcal{Q}, \Theta$ é chamado a 1-forma canônica ou de Liouville em $T^{*} \mathcal{Q}$. Temos que $w=-d \Theta$ é uma forma simplética em $T^{*} \mathcal{Q}$, em coordenadas locais.

$$
\omega=\sum_{i=1}^{n} d q_{i} \wedge d p_{i}
$$

Propriedade 2: $\operatorname{Se}(\mathcal{M}, w)$ uma variedade simplética então podemos construir uma forma simplética em $T \mathcal{M}$, que denotaremos por $\dot{w}$.

Prova: : Definamos $\bar{w}, \bar{w}: T \mathcal{M} \longrightarrow T^{*} \mathcal{M}$ tal que :

$$
\bar{w}(\mathcal{X})=w(\mathcal{X},-) \quad \mathcal{X} \in T \mathcal{M}
$$

temos que

$$
\begin{aligned}
\bar{w}(\mathcal{X}+\mathcal{Y}) & =w(\mathcal{X}+\mathcal{Y},-) \\
& =w(\mathcal{X},-)+w(\mathcal{Y},-) \\
& =\bar{w}(\mathcal{X})+\bar{w}(\mathcal{Y})
\end{aligned}
$$

e

$$
\begin{aligned}
\bar{w}(\lambda \mathcal{X}) & =w(\lambda \mathcal{X},-) \\
& =\lambda w(\mathcal{X},-) \\
& =\lambda \bar{w}(\mathcal{X})
\end{aligned}
$$

Portanto $\bar{w}$ é linear e dado que $w$ é não degenerada, podemos dizer que $\bar{w}$, define um isomorfismo de fibrados.

$T^{*} \mathcal{M}$ é um fibrado cotangente, portanto possue uma 1-forma $\Theta$ definida como na Propiedade anterior. Temos que $\bar{\omega}^{*} \Theta$ é uma 1-forma em $T \mathcal{M}$ e $d \bar{\omega}^{*} \Theta$ é uma 2 -forma simplética em $T \mathcal{M}$ a qual denotaremos por $\dot{w}$.

Calculemos $\dot{w}$ em coordenadas locais. Tomemos coordenadas locais $p_{1}, \ldots, p_{n}, q_{1}, \ldots, q_{n}$ para $\mathcal{M}$ tal que $\omega=\sum_{i=1}^{n} d p_{i} \wedge d q_{i}$. Definindo coordenadas para $T \mathcal{M}$. Seja $f: \mathcal{M} \longrightarrow \mathbb{R}$ e definamos $\dot{f}: T \mathcal{M} \longrightarrow \mathbb{R}$ por

$$
\dot{f}\left(V_{p}\right)=d f_{p}\left(V_{p}\right)
$$

Onde $V_{p} \in T \mathcal{M}\left(\pi\left(V_{p}\right)=p, \pi: T \mathcal{M} \longrightarrow \mathcal{M}\right.$, projeção $)$.

Temos que $\left(p_{1}, \ldots, p_{n}, q_{1}, \ldots, q_{n}, \dot{p_{1}}, \ldots, \dot{p_{n}}, \dot{q_{1}}, \ldots, \dot{q_{n}}\right)$ formam um sistema de coordenadas para $T \mathcal{M}, \dot{\omega}$ definida neste sistema de coordenadas tem a forma:

$$
\dot{w}=\sum_{i}^{n} d \dot{p}_{i} \wedge d q_{i}+d p_{i} \wedge d \dot{q}_{i}
$$


Proposição 2.3.1 Seja $(\mathcal{M}, w)$ uma variedade simplética. Um campo vetorial $\mathcal{X}$ : $\mathcal{M} \longrightarrow T \mathcal{M}$ é localmente um campo vetorial Hamiltoniano se e só se $\mathcal{L}_{\mathcal{X}} w=0$.

Demonstração: Temos que $\mathcal{X}$ é localmente um campo vetorial Hamiltoniano isto é existe $H: \mathcal{M} \longrightarrow \mathbb{R}$ tal que:

$$
\begin{aligned}
\mathcal{X} & =\mathcal{X}_{H} \\
\text { e } w\left(\mathcal{X}_{H},-\right)=d H \text { tal que } \iota \mathcal{X} w=\iota \mathcal{X}_{H} w & =d H \text { onde } \iota \mathcal{X} w\left(\mathcal{Y}_{1}, \ldots, \mathcal{Y}_{n}\right)=w\left(\mathcal{X}, \mathcal{Y}_{1}, \ldots, \mathcal{Y}_{n}\right) \\
\text { portanto } & d_{\iota} \mathcal{X} w
\end{aligned}
$$

$L_{\mathcal{X}} w$ é a derivada de Lie de $w$ com respeito a $\mathcal{X}$, que é dada por:

$$
\mathcal{L}_{\mathcal{X}} w=\iota_{\mathcal{X}} d \omega+d^{\iota} \mathcal{X}^{\omega}
$$

$\operatorname{com} \iota \mathcal{X} \alpha\left(\mathcal{Y}_{1}, \ldots . \mathcal{Y}_{n}\right)=\alpha\left(\mathcal{X}, \mathcal{Y}_{1}, \ldots, \mathcal{Y}_{n}\right)$. Dado que $\omega$ é fechado e por $\left(^{*}\right)$ segue que

$$
\mathcal{L}_{\mathcal{X}} \omega=0
$$

Por outro lado, assuma que $\mathcal{L}_{\mathcal{X}} \omega=0$, segue do fato que $w$ é fechada que é equivalente a $d_{\iota} \mathcal{X} \omega=0$ ou seja $\iota \mathcal{X} w$ é fechada, pelo Lema de Poincaré (veja [2]) segue-se que localmente existe uma função $H: \mathcal{M} \longrightarrow \mathbb{R}$ tal que

$$
\iota \mathcal{X} \omega=d H
$$

Isto é $w(\mathcal{X},-)=d H$, portanto $\mathcal{X}=\mathcal{X}_{H}$, ou seja $\mathcal{X}$ é localmente Hamiltoniano.

Consideremos o seguinte sistema $\Sigma(\mathcal{M}, \mathcal{W}, \mathcal{B}, f)$

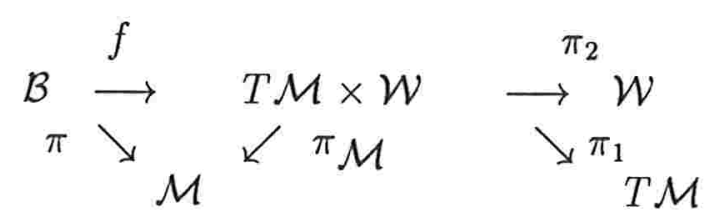

Onde:

i) $(\mathcal{M}, \omega)$ é uma variedade simplética.

ii) $\left(\mathcal{W}, \omega^{e}\right)$ é uma variedade simplética.

Por a Propiedade 2 dado que $(\mathcal{M}, \omega)$ é uma variedade simplética, podemos construir em $T \mathcal{M}$ uma forma simplética $\dot{\omega}$. Agora $T \mathcal{M} \times \mathcal{W}$ é uma variedade simplética definindo a forma $\Omega=\pi_{1}^{*} \dot{\omega}-\pi_{2}^{*} \omega^{e}$ em $T \mathcal{M} \times \mathcal{W}$. Isto é o que chamaremos um sistema Hamiltoniano de controle, no capítulo seguinte formalizaremos a definição. 
Exemplo 2.3.1 Se consideramos o sistema (4) definido no Exemplo (1.4.3). Definamos o Lagrangiano $L$ em $T(\mathcal{S O}(3))=\mathcal{S O}(3) \times \mathbb{R}^{3}$ por $L(R, w)=\frac{1}{2} w^{t} J w$. Podemos considerar Jw como um elemento de $s o^{*}=\mathbb{R}^{3}$ e (4) define um sistema Hamiltoniano com espaço estado $T^{*}(\mathcal{S O}(3)) \times \mathbb{R}^{3}$ e função Hamiltoniana $L$.

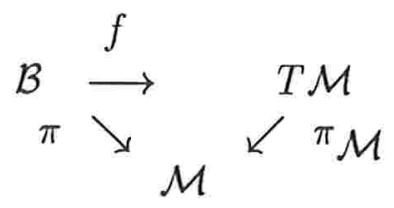

$\operatorname{Com} \mathcal{M}=T^{*}(\mathcal{S O}(3))$ e $\mathcal{B}=T^{*}(S \mathcal{O}(3)) \times \mathbb{R}^{3}$. 


\section{Capítulo 3}

\section{Sistema Hamiltoniano de Controle}

\subsection{Introdução}

O objetivo de este capítulo é definir o que entenderemos por um sistema Hamiltoniano afim de controle e demonstrar que neste tipo de sistemas temos que a propriedade de ser fortemente acessível é equivalente a fracamente observável.

Para começar veremos com um exemplo o que entenderemos por um sistema Hamiltoniano afim de controle.

Consideremos um sistema mecânico simple, o qual consiste de uma massa suspensa por uma mola a qual está sujeita a uma força externa aplicada na extremidade. A equação do movimento é dada por

$$
m \ddot{x}=m g-k(x-h)^{3}+F
$$

Onde $m, g, k$ e $h$ são constantes e $x$ é o deslocamento da massa. Se fizermos $u=F+m g, q=x-h$ e $p=m \dot{x}$ reescrevemos o sistema como:

$$
\begin{aligned}
& \dot{q}=\frac{p}{m} \\
& \dot{p}=-k q^{3}+u
\end{aligned}
$$

Se tomamos $H_{0}=\frac{p^{2}}{2 m}+\frac{k q^{4}}{4}, H_{1}=q$ e $y=q$ obtemos o seguinte sistema

$$
\begin{aligned}
& \dot{q}=\frac{\partial H_{0}}{\partial p}-u \frac{\partial H_{1}}{\partial p} \\
& \dot{p}=\frac{-\partial H_{0}}{\partial q}+u \frac{\partial H_{1}}{\partial q} \\
& y=H_{1}
\end{aligned}
$$


onde $u$ é o controle, $y$ é a saída, este é um exemplo do que chamaremos sistema Hamiltoniano.

\subsection{Subvariedade Langrangiana}

Definição 3.2.1 Uma subvariedade $\mathcal{N}$ de uma variedade simplética $(\mathcal{M}, \omega)$ é chamada Lagrangiana se:

i) $\omega(\mathcal{X}, \mathcal{Y})=0$ para todo $\mathcal{X}, \mathcal{Y} \in T \mathcal{N}$.

ii) $\mathcal{N}$ tem dimensão maximal.

Observação 3.2.1 De i) podemos deduzir que a dimensão máxima é a metade da dimensão de $\mathcal{M}$.

Exemplo 3.2.1 Seja $\mathcal{M}=T^{*} X$, $\alpha$ uma 1-forma em $\mathcal{M}$ e $w=d \alpha$, uma 2-forma simplética. Uma 1-forma em uma variedade associa um ponto da variedade, um covetor no ponto. Chamaremos gráfico de uma 1-forma fechada em $X$ a totalidade de esses covetores. O gráfico de uma 1-forma fechada em $X$, é uma subvariedade Lagrangiana de $\mathcal{M}$, na verdade a restrição da forma simplética $w=d(p d q)$ ao gráfico da 1-forma $\zeta: q \longrightarrow p(q)$ é igual a $d(p(q) d q)=d \zeta=0$, se $\zeta$ é fechado.

\subsection{Sistema Totalmente Hamiltoniano}

A definição a seguir, nos permitirá posteriormente caracterizar um sistema Hamiltoniano afim de controle.

Definição 3.3.1 Seja $(\mathcal{M}, w)$, $\left(\mathcal{W}, w^{e}\right)$ variedades simpléticas denotando o espaço de estado e saída respectivamente. Um sistema $\Sigma(\mathcal{M}, \mathcal{W}, \mathcal{B}, f)$ é chamado totalmente Hamiltoniano se $f(\mathcal{B}) \subset T \mathcal{M} \times \mathcal{W}$ é uma subvariedade Lagrangiana da variedade simplética $\left(T \mathcal{M} \times \mathcal{W}, \pi_{1}^{*} \dot{w}-\pi_{2}^{*} w^{e}\right)$ onde $\pi_{1}: T \mathcal{M} \times \mathcal{W} \longrightarrow T \mathcal{M}, \pi_{2}: T \mathcal{M} \times \mathcal{W} \longrightarrow \mathcal{W}$ são as respectivas projeções.

A seguinte Proposição nos permite descrever um sistema totalmente Hamiltoniano em forma local. 
Proposição 3.3.1 Seja $\Sigma(\mathcal{M}, \mathcal{W}, \mathcal{B}, f)$ um sistema totalmente Hamiltoniano então existem coordenadas $\left(q_{1}, \ldots, q_{n}, p_{1}, \ldots, p_{n}\right)$ para $\mathcal{M} e\left(w_{1}, \ldots, w_{2 m}\right)$ para $\mathcal{W}$ e uma função local $H\left(q_{1}, \ldots, q_{n}, p_{1}, \ldots, p_{n}, w_{1}, \ldots, w_{m}\right)$ tal que o sistema é descrito por

$$
\begin{aligned}
\dot{q}_{i} & =\frac{\partial H}{\partial p_{i}} \\
\dot{p}_{i} & =-\frac{\partial H}{\partial q_{i}} \quad i=1, \ldots, n \\
w_{m+j} & =c_{j} \frac{\partial H}{\partial w_{j}} \quad j=1, \ldots, m \quad \text { com } \quad c_{j}= \pm 1
\end{aligned}
$$

Demonstração: Temos que existem coordenadas locais para $T \mathcal{M}$.

Sejam $\left(q_{1}, \ldots, q_{n}, p_{1}, \ldots, p_{n}, \dot{q}_{1}, \ldots, \dot{q}_{n}, \dot{p_{1}}, \ldots, \dot{p}_{n}\right)$ e $\left(\tilde{w}_{1}, \ldots, \tilde{w}_{m}, \tilde{w}_{m+1}, \ldots, \tilde{w}_{2 m}\right)$ para $\mathcal{W}$ nestas coordenadas temos

$$
\Omega=\sum_{i} d \dot{q}_{i} \wedge d p_{i}-d \dot{p}_{i} \wedge d q_{i}-\sum_{j} d \tilde{w}_{j} \wedge d \tilde{w}_{m+j}
$$

$\mathcal{B}$ é um fibrado sobre $\mathcal{M}, f(\mathcal{B})$ pode ser parametrizado por $\left(q_{1}, \ldots, q_{n}, p_{1}, \ldots, p_{n}\right)$ e $m$ das coordenadas $\tilde{w}_{i} i=1, \ldots, 2 m$ de $\mathcal{W}$, não necessariamente as m-primeiras coordenadas, por isso denotemos as por $w_{1}, \ldots, w_{m}$ e o resto as denotaremos por $w_{m+1}, \ldots, w_{2 m}$ tal que se $w_{j}=w_{k}$ para $j \leq m$ então $w_{m+j}=\tilde{w}_{m+k}$ ou $\tilde{w}_{k-m}$ dependendo se $k \leq m$ ou $k>m$. Temos que $d \Omega=0$ em $T(f(\mathcal{B})$ ), pelo lema de Poincaré podemos dizer que existe localmente 1 -forma $\alpha$ tal que $\Omega=d \alpha$, como $f(\mathcal{B})$ é uma variedade Lagrangiana, portanto $\Omega=d \alpha$ restrito a $f(\mathcal{B})$ é zero. Pelo lema de Poincaré, existe uma função $H: f(\mathcal{B}) \longrightarrow \mathbb{R}$ tal que $d H=\alpha$ em $f(\mathcal{B})$. Assumamos que $\Omega=\sum_{i} d \dot{q}_{i} \wedge d p_{i}-d \dot{p}_{i} \wedge$ $d q_{i}-\sum_{j} d w_{j} \wedge d w_{m+j}$ portanto $\alpha$ pode ser escrito por $\alpha=\sum_{i} \dot{p}_{i} d q_{i}+\dot{q}_{i} d p_{i}+\sum_{j} w_{j} d w_{j+m}$ e $f(\mathcal{B})$ pode ser parametrizado por $q_{1}, \ldots, q_{n}, p_{1}, \ldots, p_{n}, w_{1}, \ldots, w_{m}$, temos que $H$ é uma função desde $\left(q_{1}, \ldots, q_{n}, p_{1}, \ldots, p_{n}, w_{1}, \ldots, w_{m}\right)$ a $\mathbb{R}$ portanto $f(\mathcal{B})$ é descrito localmente por

$$
f(\mathcal{B})=\left\{\begin{array}{l}
\left(q_{1}, \ldots, q_{n}, p_{1}, \ldots, p_{n}, w_{1}, \ldots, w_{m}, \dot{q}_{1}, \ldots, \dot{q}_{n}, \dot{p_{1}}, \ldots, \dot{p_{n}}, w_{m+1}, \ldots, w_{2 m}\right) \backslash \\
\dot{q}_{i}=\frac{\partial H}{\partial p_{i}} \quad i=1, \ldots, m \\
\dot{p}_{i}=-\frac{\partial H}{\partial q_{i}} \\
w_{m+j}=c_{j} \frac{\partial H}{\partial w_{j}} \quad j=1, \ldots, m
\end{array}\right\}
$$

O qual demonstra a Proposição.

\subsection{Sistema Hamiltoniano Afim}

Se considerarmos um sistema mecânico com n graus de liberdade, localmente representado por coordenadas $q=\left(q_{1}, \ldots, q_{n}\right)$. Temos a seguinte equação de movimento

$$
\frac{d}{d t}\left(\frac{\partial T}{\partial \dot{q}_{i}}\right)-\frac{\partial T}{\partial q}=F_{i} \quad 0 \leq i \leq n
$$


Onde $T(q, \dot{q})$ é a energia cinética, $\dot{q}=\left(\dot{q}_{1}, \ldots, \dot{q}_{n}\right)$ a velocidade generalizada, $F_{i}$ são as forças do sistema

$$
F_{i}=-\frac{\partial V(q)}{\partial q_{i}}+F_{i}^{e} \quad 1 \leq i \leq n
$$

Onde $F_{i}^{e}$ denota as forças externas dissipativas e generalizadas e o outros termos denotam as forças conservativas, isto, é as forças que vem da energia potencial, $V(q)$ denota a função de energia potencial. A função Lagrangiana vem dada por

$$
L_{0}(q, \dot{q})=T(q, \dot{q})-V(q)
$$

substituindo em (2) temos a equação de Euler-Lagrange

$$
\frac{d}{d t}\left(\frac{\partial L_{0}}{\partial \dot{q}_{i}}\right)-\frac{\partial L_{0}}{\partial q_{i}}=F_{i}^{e} \quad 1 \leq i \leq n
$$

se não consideramos as forças dissipativas e as forças externas representam o controle $u_{i}$, obtemos o sistema de controle

$$
\begin{aligned}
\frac{d}{d t}\left(\frac{\partial L_{0}}{\partial \dot{q}_{i}}\right)-\frac{\partial L_{0}}{\partial q_{i}} & =\left\{\begin{array}{cc}
u_{i} & i=1, \ldots, m \\
0 & i=m+1, \ldots, n
\end{array}\right. \\
y_{j} & =q_{j} j=1, \ldots, m
\end{aligned}
$$

Para um Lagrangiano $L(q, \dot{q}, u)$ dependendo de $u$ e em ausência de outras forças externas, temos a seguinte equação de movimento.

$$
\begin{array}{cccc}
\frac{d}{d t}\left(\frac{\partial L}{\partial \dot{q}_{i}}(q, \dot{q}, u)\right)-\frac{\partial L}{\partial q_{i}}(q, \dot{q}, u) & =0 & 1 \leq i \leq n \\
y_{i} & =q_{i}
\end{array}
$$

Chamado sistema de controle Lagrangiano.

Se definimos o momento generalizado

$$
p_{i}=\frac{\partial L}{\partial \dot{q}_{i}}(q, \dot{q}, u) \quad 1 \leq i \leq n
$$

Definimos a função Hamiltoniana $H(q, p, u)$ como a transformada de Legendre de $L(q, \dot{q}, u)$ ou seja

$$
\begin{aligned}
H(q, p, u) & =\sum_{i=1}^{n} p_{i} \dot{q}_{i}-L(q, \dot{q}, u) \\
& =\sum_{i=1}^{n} \frac{\partial L(q, \dot{q}, u)}{\partial \dot{q}_{i}} \dot{q}_{i}-L(q, \dot{q}, u)
\end{aligned}
$$


Derivando em relação a $p_{i}$ e substituindo a relação (5) em (4), obtemos a equação Hamiltoniana de movimento

$$
\begin{aligned}
\dot{q}_{i} & =\frac{\partial H}{\partial p_{i}}(q, p, u) \quad 1 \leq i \leq n \\
\dot{p}_{i} & =-\frac{\partial H}{\partial q_{i}}(q, p, u)+u_{i} \quad i=1, \ldots m \\
\dot{p}_{i} & =-\frac{\partial H}{\partial q_{i}}(q, p, u) \quad i=m+1, \ldots, n \\
y_{j} & =q_{j} \quad j=1, \ldots m
\end{aligned}
$$

O qual chamaremos um sistema Hamiltoniano de controle, onde $\mathrm{H}$ representa a energia do sistema, uma natural saída é definida por

$$
y_{j}=-\frac{\partial H}{\partial u_{j}}(q, p, u)
$$

Definição 3.4.1 Um sistema Hamiltoniano afim de controle é um sistema Hamiltoniano de controle com uma função Hamiltoniana da forma

$$
H(q, p, u)=H_{0}(q, p)-\sum_{j=1}^{m} H_{j}(q, p) u_{j}
$$

isto é

$$
\begin{aligned}
\dot{q}_{i} & =\frac{\partial H_{0}}{\partial p_{i}}(q, p)-\sum_{j=1}^{m} \frac{\partial H_{j}}{\partial p_{i}}(q, p) u_{j} \quad 1 \leq i \leq n \\
\dot{p_{i}} & =-\frac{\partial H_{0}}{\partial q_{i}}(q, p)+\sum_{j=1}^{m} \frac{\partial H_{j}}{\partial q_{i}}(q, p) u_{j} \\
y_{j} & =H_{j}(q, p) \quad 1 \leq j \leq m .
\end{aligned}
$$

As equações acima constituem uma generalização das equações de Euler-Lagrange.

Se tomarmos

$$
L(q, \dot{q}, u)=L_{0}(q, \dot{q})+\sum_{j=1}^{m} q_{j} u_{j}
$$

O sistema (3) pode ser visto como um caso especial da definição (3.4.1) e segue que

$$
H(q, p, u)=H_{0}(q, p)-\sum_{j=1}^{m} q_{j} u_{j}
$$


Onde $H_{0}(q, p)$ é a transformada de Legendre de $L_{0}(q, \dot{q})$ e temos que $\frac{\partial H_{j}}{\partial u_{j}}=-q_{j}$ isto é $y_{j}=-q_{j}$. O que nos da uma dualidade entre a saída e a entrada, se $u_{1}, \ldots, u_{m}$ são as forças externas generalizadas então $y_{1}, \ldots, y_{m}$ são as coordenadas generalizadas de configuração. Agora, vejamos uma definição mais global.

Definição 3.4.2 Seja $\mathcal{M}$ uma variedade com colchete de Poisson não-degenerado, $H_{0}, H_{1}, \ldots, H_{m} \in C^{\infty}(\mathcal{M})$ então

$$
\begin{aligned}
& \dot{x}=X_{H_{0}}(x)-\sum_{j=1}^{m} X_{H_{j}}(x) u_{j} \quad x \in \mathcal{M} \\
& y_{j}=H_{j}(x) \quad 1 \leq j \leq m
\end{aligned}
$$

é um sistema Hamiltoniano afim de controle.

Vejamos a equivalência com a outra definição. Temos pelo Teorema (2.2.1) que em torno de qualquer $x \in \mathcal{M}$, existem coordenadas canônicas $x=\left(q_{1}, \ldots, q_{n}, p_{1}, \ldots, p_{n}\right)$ de $\mathcal{M}$ tal que:

$$
\begin{aligned}
& X_{H_{0}}\left(q_{i}\right)=\left\{H_{0}, q_{i}\right\}=\frac{\partial H_{0}}{\partial p_{i}} \\
& X_{H_{j}}\left(q_{i}\right)=\left\{H_{j}, q_{i}\right\}=\frac{\partial H_{j}}{\partial p_{i}} \\
& X_{H_{o}}\left(p_{i}\right)=\left\{H_{o}, p_{i}\right\}=-\frac{\partial H_{0}}{\partial q_{i}} \\
& X_{H_{j}}\left(p_{i}\right)=\left\{H_{j}, p_{i}\right\}=-\frac{\partial H_{j}}{\partial q_{i}} .
\end{aligned}
$$

Substituindo o sistema da definição (3.4.2) obtemos

$$
\begin{aligned}
& \dot{q}_{i}=\frac{\partial H_{0}}{\partial p_{i}}-\sum_{j=1}^{m} \frac{\partial H_{j}}{\partial p_{i}} u_{j} \quad 1 \leq i \leq n \\
& \dot{p}_{i}=-\frac{\partial H_{0}}{\partial q_{i}}+\sum_{j=1}^{m} \frac{\partial H_{j}}{\partial q_{i}} u_{j} \\
& y_{j}=H_{j}(q, p) \quad 1 \leq j \leq m
\end{aligned}
$$

que é o sistema definido em (3.4.1).

Agora Vejamos outra definição de sistema Hamiltoniano afim de controle. 
Definição 3.4.3 Um sistema afim em uma variedade simplética $(\mathcal{M}, w)$ é chamado de sistema Hamiltoniano afim de controle se em coordenadas locais pode ser representado por

$$
\begin{aligned}
\dot{x} & =f(x)+\sum_{i=1}^{m} u_{i} g_{i}(x) \\
y_{i} & =h_{i}(x) \quad 1 \leq i \leq m
\end{aligned}
$$

Onde $f$ é localmente um campo vetorial Hamiltoniano ou seja $\mathcal{L}_{f} w=0$ e com $g_{i}$ campo vetorial Hamiltoniano tal que $w\left(g_{i},-\right)=d h_{i}$.

Temos que $f$ e $g_{i}$ são campos Hamiltonianos ou seja existem $H_{0}, H_{i} \in C^{\infty}(\mathcal{M})$ tal que $f=X_{H_{0}}$ e $g_{i}=X_{H_{i}}$, por outro lado $w\left(g_{i},-\right)=w\left(X_{H_{i}},-\right)=d H_{i}=d h_{i} \quad i=1, \ldots, m$ , portanto

$$
\begin{aligned}
& \dot{x}=X_{H_{0}}+\sum_{i=1}^{m} u_{i} X_{H_{i}} \\
& y_{i}=H_{i}(x) \quad 1 \leq i \leq m
\end{aligned}
$$

que é nosso sistema da definição (3.4.3).

Este tipo de sistemas Hamiltonianos forma uma subclasse natural da classe de sistemas dados na Definição (3.3.1), que está fundamentado pelo seguinte Teorema.

Teorema 3.4.1 Seja $\Sigma(\mathcal{M}, \mathcal{W}, \mathcal{B}, f)$ um sistema totalmente Hamiltoniano. Denotemos $f=(g, h)$ com $g: \mathcal{B} \longrightarrow T \mathcal{M}$ e $h: \mathcal{B} \longrightarrow \mathcal{W}$. Suponhamos que $\mathcal{B}$ e $\mathcal{W}$ são fibrados vetoriais, tal que $\mathcal{W}=T^{*} \mathcal{Y}$ com $\mathcal{Y}$ a variedade de saida. $\left(T^{*} \mathcal{Y}, w^{e}\right)$ é uma variedade simplética. Suponhamos também que $h: \mathcal{B} \longrightarrow T^{*} \mathcal{Y}$ é um morfismo de fibrados e que $h$ é linear e bijetora. Então podemos encontrar campos vetoriais $A e$ $B_{i} \quad i=1, \ldots, m(\operatorname{Dim} \mathcal{B}=m)$ e uma transformação $C: \mathcal{M} \longrightarrow \mathcal{Y}$ tal que o sistema é escrito localmente por:

$$
\begin{aligned}
\dot{x} & =A(x)+\sum_{i=1}^{m} u_{i} B_{i}(x) \\
y_{i} & =C_{i}(x) \quad i=1, \ldots, m
\end{aligned}
$$

$\operatorname{com}\left(y_{1}, \ldots, y_{m}\right)$ coordenadas para $\mathcal{Y}$ e $C=\left(C_{1}, \ldots, C_{m}\right)$ tal que $\mathcal{L}_{A} w=0$ e w $\left(B_{i},-\right)=$ $d C_{i}$ isto é um sistema Hamiltoniano afim de controle. 
Demonstração: Em coordenadas locais $x$ para $\mathcal{M}, u$ para o fibrado de $\mathcal{B}, w$ para $\mathcal{W}$ e $f=(g, h)$ o sistema é dado por

$$
\begin{aligned}
\dot{x} & =g(x, u) \\
w & =h(x, u),
\end{aligned}
$$

onde $g: \mathcal{B} \longrightarrow T \mathcal{M}$ e $h: \mathcal{B} \longrightarrow \mathcal{W}$ são transformações suaves. Dado $\Delta(x)$ um subespaço afim de $T_{x} \mathcal{M}$ para todo $x$ e uma transformação $h: \mathcal{M} \longrightarrow \mathcal{Y}$ onde $\mathcal{Y}$ é uma variedade de saída. Em coordenadas locais um sistema afim de controle não linear é representado por:

$$
\begin{aligned}
\dot{x} & =f(x)+\sum_{j=1}^{m} g_{j}(x) u_{j} \quad(*)(*) \\
y_{j} & =h_{j}(x) \quad j=1, \ldots p
\end{aligned}
$$

Com $x=\left(x_{1}, \ldots, x_{n}\right)$ coordenadas para $\mathcal{M}$ e $y=\left(y_{1}, \ldots, y_{p}\right)$ coordenadas para $\mathcal{Y}$, $u=\left(u_{1}, \ldots, u_{m}\right) \in \mathcal{U}, f, g_{1}, \ldots, g_{m}$ são campos vetoriais suaves definidos em $\mathcal{M}$ tal que $\Delta(x)=f(x)+\operatorname{Span}\left\{g_{1}(x), \ldots, g_{m}(x)\right\}$. Da Proposição (3.3.1), para cada u fixado $f(x, u)$ é um campo vetorial Hamiltoniano e em cada $x_{0} \in \mathcal{M}$ existe uma vizinhança $V$ de $x_{0}$ tal que em $V$, podemos escrever (*) na forma

$$
\begin{aligned}
\dot{x} & =\mathcal{X}_{H_{u}}(x) \\
y & =\frac{\partial H^{t}}{\partial u}(x, u)
\end{aligned}
$$

Onde $x \in V \subset \mathcal{M}, \quad u \in \mathcal{U} \subset \mathbb{R}^{m}, y \in \mathbb{R}^{m}$. Isto é, em $V$ para cada $u$ fixado $\mathcal{X}_{H_{u}}$ é um campo vetorial Hamiltoniano, com função Hamiltoniana $x \longrightarrow H_{u}(x)=H(x, u)$, e $w\left(\mathcal{X}_{H_{u}}, \mathcal{Z}\right)=d H_{u}(\mathcal{Z})$ para todo campo vetorial $\mathcal{Z}$ em $V$. Se o sistema é do tipo $(*)(*)$, podemos escrever este sistema da forma:

$$
\begin{array}{rlrl}
\dot{x} & =g_{0}-\sum_{i=1}^{m} u_{i} \mathcal{X}_{H_{i}}(x) & & x \in \mathcal{M} \\
y_{i} & =H_{i}(x) & & \\
& 1 \leq i \leq m \quad y \in \mathbb{R}^{m}
\end{array}
$$

Onde $\mathcal{X}_{H_{i}}$ são os campos vetoriais Hamiltonianos globais com função Hamiltoniana $H_{i}$, e $g_{0}$ é um campo vetorial Hamiltoniano local, com uma função Hamiltoniana definida localmente $H_{0}$, e $w\left(\mathcal{X}_{H_{i}},-\right)=d H_{i}$. Tomando $A=g_{0}$ e dado que $g_{0}$ é um campo vetorial Hamiltoniano local, temos que $\mathcal{X}_{H_{i}}=B_{i}$, sendo $C_{i}=H_{i}$ e além disso, $w\left(B_{i},-\right)=w\left(\mathcal{X}_{H_{i}},-\right)=d H_{i}=d C_{i}$. O qual demonstra o Teorema.

\subsection{Acessibilidade e Observabilidade}

A seguir veremos uma definição equivalente à dada no Capítulo 1 relativa ao conceito de fortemente acessível. 
Definição 3.5.1 Seja o sistema (1). $\left.\Gamma_{0}=<g_{1}, \ldots, g_{m}\right\rangle$ denota o subespaço vetorila de $V^{\infty}(\mathcal{M})$ gerado por $\left\{g_{1}, \ldots, g_{m}\right\}$ e $\Gamma=f+\left\langle g_{1}, \ldots, g_{m}\right\rangle$ (um subespaço afim de $\left.V^{\infty}(\mathcal{M})\right)$. Definamos $\Gamma_{k}=\left[\Gamma, \Gamma_{k-1}\right]+\Gamma_{k-1} \quad k \geq 1$. Seja $K=\bigcup_{k}>{ }_{0} \Gamma_{k}$. O sistema é chamado fortemente acessivel se $K(x)=T_{x} \mathcal{M}$ para todo $x \in \mathcal{M}$, onde $K(x)$ é o subespaço vetorial de $T_{x} \mathcal{M}$ gerado por os campos vetoriais em $K$.

Para demonstrar a equivalencia com o Teorema (1.3.2), nos basta demonstrar que $K(x)=C_{0}(x)$ para todo $x \in \mathcal{M}$. Da Definição (1.3.7) e Proposição (1.3.3), concluimos que $K \subset \mathcal{C}_{0}$. Se demonstramos que $K$ é um subálgebra de Lie, por definição de $\mathcal{C}_{0}$ teremos que $K=C_{0}$. Por isso, basta demonstrar que $K$ é uma álgebra de Lie.

Temos que,

$$
\begin{aligned}
{\left[\Gamma_{0}, \Gamma_{n}\right] } & =\left[f+\Gamma_{0}-f, \Gamma_{n}\right] \\
& =\left[f+\Gamma_{0}, \Gamma_{n}\right]+\left[f,-\Gamma_{n}\right] \\
& =\left[\Gamma, \Gamma_{n}\right]+\left[f,-\Gamma_{n}\right] \\
& =\left[\Gamma, \Gamma_{n}\right]+\Gamma_{n+1} \\
& =\Gamma_{n+2} \quad \forall n
\end{aligned}
$$

Por outro lado,

$$
\begin{aligned}
{\left[\Gamma, \Gamma_{n}\right] } & =\left[\Gamma, \Gamma_{n}\right]+\Gamma_{n} \quad \text { onde o ultimo } \Gamma_{n}=0 \\
& =\Gamma_{n+1} \quad \forall n .
\end{aligned}
$$

Demonstremos que $\left[\Gamma_{p}, \Gamma_{n}\right] \in K$, para isso aplicaremos indução sobre $p$. Do anterior temos que $\left[\Gamma_{0}, \Gamma_{n}\right] \in K$. Vejamos se é válido para $p=1$

$$
\begin{aligned}
{\left[\Gamma_{1}, \Gamma_{n}\right] } & =\left[\left[\Gamma, \Gamma_{0}\right]+\Gamma_{0}, \Gamma_{n}\right] \\
& =\left[\left[\Gamma, \Gamma_{0}\right], \Gamma_{n}\right]+\left[\Gamma_{0}, \Gamma_{n}\right] \\
& =-\left[\left[\Gamma_{0}, \Gamma_{n}\right], \Gamma\right]-\left[\left[\Gamma_{n}, \Gamma\right], \Gamma_{0}\right]+\left[\Gamma_{0}, \Gamma_{n}\right] \quad \text { pela identidade de Jacobi } \\
& =\left[\Gamma, \Gamma_{n+2}\right]+\Gamma_{n+3}+\Gamma_{n+2} \\
& =\left[\Gamma, \Gamma_{n+2}\right]+\Gamma_{n+3} \\
& =\Gamma_{n+4}
\end{aligned}
$$

Portanto $\left[\Gamma_{1}, \Gamma_{n}\right] \in K$ para todo $n$. Suponhamos que é válido para $p=k$ e para todo $n$, Vou demonstrar que é válido para $p=k+1$.

$$
\begin{array}{rlr}
{\left[\Gamma_{k+1}, \Gamma_{n}\right]} & =\left[\left[\Gamma, \Gamma_{k}\right]+\Gamma_{k}, \Gamma_{n}\right] & \\
& =\left[\left[\Gamma, \Gamma_{k}\right], \Gamma_{n}\right]+\left[\Gamma_{k}, \Gamma_{n}\right] & \\
& =-\left[\left[\Gamma_{k}, \Gamma_{n}\right], \Gamma\right]-\left[\left[\Gamma_{n}, \Gamma\right], \Gamma_{k}\right]+\left[\Gamma_{k}, \Gamma_{n}\right] & \text { pela identidade de Jacobi } \\
& =-\left[\Gamma_{k+n+2}, \Gamma\right]-\left[\Gamma_{n+1}, \Gamma_{k}\right]+\left[\Gamma_{k}, \Gamma_{n}\right] & \\
& =\left[\Gamma, \Gamma_{k+n+2}\right]+\left[\Gamma_{k}, \Gamma_{n+1}\right]+\left[\Gamma_{k}, \Gamma_{n}\right] .
\end{array}
$$


Temos que os três termos do lado direito pertencem a $K$, e a suma também pertence a $K$. Portanto

$$
\left[\Gamma_{k+1}, \Gamma_{n}\right] \in K
$$

e, portanto,

$$
\left[\Gamma_{p}, \Gamma_{n}\right] \in K
$$

Isso mostra a equivalencia com o Teorema (1.3.2).

Agora damos uma definição equivalente á dada no Capítulo 1 de localmente fracamente observável.

Definição 3.5.2 Seja o sistema (1) e definamos $F_{k}=\mathcal{L}_{\Gamma} F_{k-1}+F_{k-1} \quad k \geq 1$, $\Gamma$ como a definição anterior e $F_{0}=<h_{1}, \ldots, h_{m}>$. Então o sistema é localmente fracamente observável se $G=\bigcup_{k}>{ }_{0} F_{k}$ satisfaz $d G(x)=T_{x}^{*} \mathcal{M}$ para todo $x \in \mathcal{M}$, onde $d G(x)$ é o subespaço vetorial de $T^{*} \mathcal{M}$ gerado por $d h(x)$ com $h \in G$.

Da Proposição(1.4.1) temos que $G=\bigcup_{k}>{ }_{0} F_{k}$ é equivalente à definição do espaço de observação $\mathcal{O}$, e nosso critério é equivalente ao Teorema(1.4.1). Vejamos que sucede se nosso sistema é um sistema Hamiltoniano afim de controle, lembremos que neste tipo de sistemas temos que se $\mathcal{L}_{f} w=0$, existe $H \in C(\mathcal{M})$ tal que $f=\mathcal{X}_{H}$ e se $g_{i}$ é campo vetorial Hamiltoniano, existe $H_{i} \in C(\mathcal{M})$ tal que $g_{i}=\mathcal{X}_{H_{i}}$ e $w\left(g_{i},-\right)=d h_{i}$ temos que $H_{i}=h_{i}$ a menos de uma constante. Temos, então, a seguinte Proposição.

Proposição 3.5.1 Seja $F=H+<h_{1}, \ldots, h_{m}>$. Então os $F_{k}$ definidos anteriormente satisfazen $F_{k}=\left\{F, F_{k-1}\right\}+F_{k-1} \operatorname{com}\{\cdot, \cdot\}$ o colchete do Poisson.

Demonstração: Da definição(1.4.7) temos que os $F_{k}$ são soma de funções da forma

$$
\mathcal{L}_{\mathcal{X}_{1}} \mathcal{L}_{\mathcal{X}_{2}} \ldots \mathcal{L}_{\mathcal{X}_{k}} h_{j}
$$

$\operatorname{com} \mathcal{X}_{i}=f$ ou $\mathcal{X}_{i}=g_{l}=\mathcal{X}_{H_{i}}$ para $l=1, \ldots, m$. O colchete de Poisson é definido por

$$
\{A, B\}=w\left(\mathcal{X}_{B}, \mathcal{X}_{A}\right)=\mathcal{X}_{A}(B)
$$

temos que $\mathcal{L}_{\mathcal{X}_{i}} h_{j}=\left\{H, h_{j}\right\}$ se $\mathcal{X}_{i}=f$ e $\mathcal{L} \mathcal{X}_{i} h_{j}=\left\{h_{l}, h_{j}\right\}$ se $\mathcal{X}_{i}=\mathcal{X}_{H_{l}}$, generalizando obtemos

$$
\mathcal{L}_{\mathcal{X}_{1}} \mathcal{L}_{\mathcal{X}_{2}} \ldots \mathcal{L}_{\mathcal{X}_{k}} h_{j}=\left\{c_{1},\left\{c_{2},\left\{c_{3}, \ldots\left\{c_{k}, h_{j}\right\} \ldots\right\}\right\}\right\}
$$

Com $c_{i}=H$ ou $c_{i}=h_{l}, \quad l=1, \ldots, m$ com isto fica provada nossa proposição. 
Vejamos se $G$, definido em um sistema Hamiltoniano afim de controle é uma álgebra de Lie.

Temos que

$$
\begin{aligned}
\left\{F_{0}, F_{n}\right\} & =\left\{H+F_{0}-H, F_{n}\right\} \\
& =\left\{H+F_{0}, F_{n}\right\}+\left\{H,-F_{n}\right\} \\
& =\left\{F, F_{n}\right\}+F_{n+1} \\
& =F_{n+2} \quad \forall n .
\end{aligned}
$$

Por outro lado

$$
\begin{aligned}
\left\{F, F_{n}\right\} & =\left\{F, F_{n}\right\}+F_{n} \quad \text { onde o ultimo } F_{n}=0 \\
& =F_{n+1} \forall n .
\end{aligned}
$$

demonstremos que $\left\{F_{p}, F_{n}\right\} \in G$, para isso aplicaremos indução sobre p. Do anterior temos que $\left\{F_{0}, F_{n}\right\} \in G$.

Vejamos se é válido para $p=1$.

$$
\begin{aligned}
\left\{F_{1}, F_{n}\right\} & =\left\{\left\{F, F_{0}\right\}+F_{0}, F_{n}\right\} \\
& =\left\{\left\{F, F_{0}\right\}, F_{n}\right\}+\left\{F_{0}, F_{n}\right\} \\
& =-\left\{\left\{F_{0}, F_{n}\right\}, F\right\}-\left\{\left\{F_{n}, F\right\}, F_{0}\right\}+F_{n+2} \quad \text { (pela identidade de Jacobi) } \\
& =\left\{F,\left\{F_{0}, F_{n}\right\}\right\}+\left\{F_{0},-\left\{F, F_{n}\right\}\right\}+F_{n+2} \\
& =\left\{F, F_{n+2}\right\}+\left\{F_{0}, F_{n+1}\right\}+F_{n+2} \\
& =\left\{F, F_{n+2}\right\}+F_{n+3}+F_{n+2} \\
& =\left\{F, F_{n+2}\right\}+F_{n+3} \\
& =F_{n+4}
\end{aligned}
$$

Portanto $\left\{F_{1}, F_{n}\right\} \in G$ para todo $n$. Suponhamos que é válido para $p=k$ e para todo $n$, vou a demonstrar que é válido para $p=k+1$.

$$
\begin{aligned}
\left\{F_{k+1}, F_{n}\right\} & =\left\{\left\{F, F_{k}\right\}+F_{k}, F_{n}\right\} \\
& =\left\{\left\{F, F_{k}, F_{n}\right\}+\left\{F_{k}, F_{n}\right\}\right. \\
& =-\left\{\left\{F_{k}, F_{n}\right\}, F\right\}-\left\{\left\{F_{n}, F\right\}, F_{k}\right\}+\left\{F_{k}, F_{p}\right\} \\
& =\left\{F,\left\{F_{k}, F_{p}\right\}\right\}+\left\{F_{k},\left\{F_{n}, F\right\}\right\}+\left\{F_{k}, F_{p}\right\} \\
& =\left\{F, F_{k+p+2}\right\}+\left\{F_{k}, F_{n+1}\right\}+\left\{F_{k}, F_{n+1}\right\}+\left\{F_{k}, F_{p}\right\}
\end{aligned}
$$

Temos que os três termos do lado direito pertencem a $G$ e a soma também pertence a $G$. Portanto

$$
\left\{F_{k+1}, F_{n}\right\} \in G
$$

$\mathrm{e}$

$$
\left\{F_{p}, F_{n}\right\} \in G
$$

Isso mostra que $\mathrm{G}$ é uma álgebra de Lie.

Relacionemos os $F_{k}$ e $\Gamma_{k}$ através de un isomorfismo, através do seguinte Teorema. 
Teorema 3.5.1 Dado um sistema Hamiltoniano afim de controle. Seja $\Gamma_{k}$ e $F_{k} d a$ forma definidos anteriormente. Então $\Gamma_{k}$ e $F_{k}$ são relacionados pela transformação

$$
N \stackrel{\alpha}{\longrightarrow} \mathcal{X}_{N}
$$

(definido por $\left.w\left(\mathcal{X}_{N},-\right)=d N\right)$ é um isomorfismo entre as álgebras de Lie $G$ e $K$.

Demonstração: Temos que a transformação $\alpha$ associa funções constantes ao campo vetorial nulo. Para a demonstração usaremos indução sobre $k$.

Provaremos que a afirmação é válida para $k=0$.

$$
\begin{aligned}
& F_{0}=<h_{1}, \ldots, h_{m}>\text { e } \Gamma_{0}=<g_{1}, \ldots, g_{m}>\text {, seja } N=\sum_{i=1}^{m} \beta_{i} h_{i} \in F_{0}, \quad \beta_{i} \in \mathbb{R} \\
& \alpha(N)=\alpha\left(\sum_{i=1}^{m} \beta_{i} h_{i}\right)=\mathcal{X}_{\sum_{i=1}^{m} \beta_{i} h_{i}}=\sum_{i=1}^{m} \beta_{i} \mathcal{X}_{h_{i}}=\sum_{i=1}^{m} \beta_{i} g_{i} \in \Gamma_{0} .
\end{aligned}
$$

Portanto $F_{0}$ é associado a $\Gamma_{0}$.

Vejamos agora se é válido para $k=1$. Temos $F_{1}=\left\{F, F_{0}\right\}+F_{0}$

$$
\begin{aligned}
\alpha\left(F_{1}\right) . & =\alpha\left(\left\{F, F_{0}\right\}\right)+\alpha\left(F_{0}\right) \\
& =\alpha\left(\left\{H+F_{0}, F_{0}\right\}+\Gamma_{0}\right. \\
& =\alpha\left(\left\{H, F_{0}\right\}+\left\{F_{0}, F_{0}\right\}\right)+\Gamma_{0} \\
& =\alpha\left(\left\{H, F_{0}\right\}\right)+\alpha\left(\left\{F_{0}, F_{0}\right\}\right)+\Gamma_{0} \\
& =\left[\mathcal{X}_{H}, \mathcal{X}_{F_{0}}\right]+\left[\mathcal{X}_{F_{0}}, \mathcal{X}_{F_{0}}\right]+\Gamma_{0} \\
& =\left[f, \alpha\left(F_{0}\right)\right]+\left[\alpha\left(F_{0}\right), \alpha\left(F_{0}\right)\right]+\Gamma_{0} \\
& =\left[f, \Gamma_{0}\right]+\left[\Gamma_{0}, \Gamma_{0}\right]+\Gamma_{0} \\
& =\left[f+\Gamma_{0}, \Gamma_{0}\right]+\Gamma_{0} \\
& =\left[\Gamma, \Gamma_{0}\right]+\Gamma_{0} \\
& =\Gamma_{1}
\end{aligned}
$$


Portanto é válido para $k=1$. Suponhamos que é válida para $k-1$ e provemos que é válida para $k$ Temos

$$
F_{k}=\left\{F, F_{k-1}\right\}+F_{k-1}
$$

Por hipótese de indução temos que $\alpha\left(F_{k-1}\right)=\Gamma_{k-1}$, calculemos $\alpha\left(\left\{F, F_{k-1}\right\}\right)$.

$$
\begin{aligned}
\alpha\left(\left\{F, F_{k-1}\right\}\right) & =\alpha\left(\left\{H+F_{0}, F_{k-1}\right)\right. \\
& =\alpha\left(\left\{H, F_{k-1}\right\}+\left\{F_{0}, F_{k-1}\right\}\right) \\
& =\alpha\left(\left\{H, F_{k-1}\right\}\right)+\alpha\left(\left\{F_{0}, F_{k-1}\right\}\right) \\
& =\left[X_{H}, X_{F_{k-1}}\right]+\left[X_{F_{0}}, X_{F_{k-1}}\right] \\
& =\left[f, \alpha\left(F_{k-1}\right)\right]+\left[\alpha\left(F_{0}\right), \alpha\left(F_{k-1}\right)\right] \\
& =\left[f, \Gamma_{k-1}\right]+\left[\Gamma_{0}, \Gamma_{k-1}\right] \\
& =\left[f+\Gamma_{0}, \Gamma_{k-1}\right] \\
& =\left[\Gamma, \Gamma_{k-1}\right]
\end{aligned}
$$

Portanto

$$
\alpha\left(\left\{F, F_{k-1}\right\}\right)=\left[\Gamma, \Gamma_{k-1}\right]
$$

Do anterior temos que

$$
\begin{aligned}
\alpha\left(F_{k}\right) & =\alpha\left(\left\{F, F_{k-1}\right\}+F_{k-1}\right) \\
& =\alpha\left(\left\{F, F_{k-1}\right\}\right)+\alpha\left(F_{k-1}\right) \\
& =\left[\Gamma, \Gamma_{k-1}\right]+\Gamma_{k-1} \\
& =\Gamma_{k}
\end{aligned}
$$

Portanto temos que é válido para k. O qual demonstra nosso Teorema.

Corolário 3.5.1 Um sistema Hamiltoniano afim de controle é localmente fracamente observável se e só se é fortemente acessivel.

Demonstração: Do Teorema anterior temos que $G=\bigcup_{k} \geq{ }_{0} F_{k}$ é transformado por $\alpha$ isomorficamente em $K=\bigcup_{k} \geq{ }_{0} \Gamma_{k}$ isto é: 


$$
\begin{aligned}
\alpha(G) & =\alpha\left(\bigcup_{k} \geq 0{ }_{0} F_{k}\right) \\
& =\bigcup_{k} \geq{ }_{0} \alpha\left(F_{k}\right) \\
& =\bigcup_{k} \geq 0 \Gamma_{k} \\
& =K
\end{aligned}
$$

Portanto $d G(x)=T_{x}^{*} \mathcal{M}$ se, e só se, $K(x)=T_{x} \mathcal{M}$.

O qual demonstra que um sistema Hamiltoniano afim de controle é controlável se, e só se, é observável, no sentido que fortemente acessivel implica localmente fracamente observável e vice-versa.

Exemplo 3.5.1 Voltando ao Exemplo 1.2.1. denotemos $\theta_{1}=q_{1} \theta_{2}=q_{2}$. A energia cinética vem dada por

$$
T(q, \dot{q})=\frac{1}{2} \dot{q}^{t} A(q) \dot{q}
$$

Se tomamos $l_{1}=l_{2}=1, m_{1}=m_{2}=1$

$$
A(q)=\left(\begin{array}{cc}
3+2 \cos q_{2} & 1+\cos q_{2} \\
1+\cos q_{2} & 1
\end{array}\right)
$$

Portanto

$$
\begin{aligned}
T(q, \dot{q}) & =\frac{1}{2}\left(\dot{q}_{1} \dot{q}_{2}\right)\left(\begin{array}{cc}
3+2 \cos q_{2} & 1+\cos q_{2} \\
1+\cos q_{2} & 1
\end{array}\right)\left(\begin{array}{c}
\dot{q}_{1} \\
\dot{q}_{2}
\end{array}\right) \\
& =\frac{1}{2}\left[\left(3+2 \cos q_{2}\right) \dot{q}_{1}{ }^{2}+2\left(1+\cos q_{2}\right) \dot{q}_{1} \dot{q}_{2}+\dot{q}_{2}{ }^{2}\right]
\end{aligned}
$$

O momento generalizado é

$$
\begin{aligned}
p_{i} & =\frac{\partial L}{\partial \dot{q}_{i}}(q, \dot{q}, u) \\
& =\frac{\partial\left(T(q, \dot{q})-V(q)+\sum_{j=1}^{2} q_{j} u_{j}\right)}{\partial \dot{q}} \\
& =\frac{\partial T(q, \dot{q})}{\partial \dot{q}_{i}}
\end{aligned}
$$


Temos que

$$
\begin{gathered}
p_{1}=\left(3+2 \cos q_{2}\right) \dot{q}_{1}+\left(1+\cos q_{2}\right) \dot{q}_{2} \\
p_{2}=\left(1+\cos q_{2}\right) \dot{q}_{1}+\dot{q}_{2}
\end{gathered}
$$

O Hamiltoniano é dado por

$$
H(q, p, u)=H_{0}(q, p)-\sum_{j=1}^{2} q_{j} u_{j}
$$

Mas

$$
\begin{aligned}
H_{0}(q, p)= & \frac{1}{2} p^{t} A^{-1}(q) p+V(q) \\
= & \frac{1}{2}\left(p_{1} p_{2}\right) \frac{1}{1+\sin ^{2} q_{2}}\left(\begin{array}{cc}
1 & -\left(1+\cos q_{2}\right) \\
-\left(1+\cos q_{2}\right) & 3+2 \cos q_{2}
\end{array}\right)\left(\begin{array}{l}
p_{1} \\
p_{2}
\end{array}\right) \\
& -g \cos q_{1}-g\left(\cos q_{1}+\cos \left(q_{1}+q_{2}\right)\right) \\
= & \frac{1}{2\left(1+\sin q_{2}\right)}\left[p_{1}^{2}-2 p_{1} p_{2}\left(1+\cos q_{2}\right)+p_{2}^{2}\left(3+2 \cos q_{2}\right)\right] \\
& -g\left(2 \cos q_{1}+\cos \left(q_{1}+q_{2}\right)\right.
\end{aligned}
$$

Portanto

$$
\begin{aligned}
H(q, p, u)= & \frac{1}{2\left(1+\sin ^{2} q_{2}\right)}\left[p_{1}^{2}-2 p_{1} p_{2}\left(1+\cos q_{2}\right)+p_{2}^{2}\left(3+2 \cos q_{2}\right)\right] \\
& -g\left(2 \cos q_{1}+\cos \left(q_{1}+q_{2}\right)\right)-q_{1} u_{1}-q_{2} u_{2}
\end{aligned}
$$

Do qual temos

$$
\begin{aligned}
\dot{q}_{1}= & \frac{p_{1}-p_{2}\left(1+\cos q_{2}\right)}{1+\sin q_{2}^{2}} \\
\dot{q_{2}}= & \frac{\left[-p_{1}\left(1+\cos q_{2}\right)+p_{2}\left(3+\cos q_{2}\right)\right]}{\left(1+\sin q_{2}^{2}\right)} \\
\dot{p_{1}=} & g\left(-2 \sin q_{1}-\sin \left(q_{1}+q_{2}\right)\right)+u_{1} \\
\dot{p_{2}=} & \frac{\sin q_{2} \cos q_{2}\left[p_{1}^{2}-2 p_{1} p_{2}\left(1+\cos q_{2}\right)+p_{2}^{2}\left(3+2 \cos q_{2}\right)\right]}{\left(1+\sin ^{2} q_{2}\right)^{2}} \\
& +\frac{\left[-p_{1} p_{2} \sin q_{2}+p_{2}^{2} \sin q_{2}\right]}{\left(1+\sin ^{2} q_{2}\right)}-g \sin \left(q_{1}+q_{2}\right)+u_{2}
\end{aligned}
$$


Temos o seguinte sistema:

$$
\begin{aligned}
\frac{d}{d t}\left(\begin{array}{l}
q_{1} \\
q_{2} \\
p_{1} \\
p_{2}
\end{array}\right)= & \left(\begin{array}{c}
\frac{1}{1+\sin ^{2} q_{2}}\left[-p_{1}-p_{2}\left(1+\cos q_{2}\right]\right. \\
\frac{1}{1+\sin ^{2} q_{2}}\left[-p_{1}\left(1+\cos q_{2}\right)+p_{2}\left(3+\cos q_{2}\right)\right] \\
g\left(-2 \sin q_{1}-\sin \left(q_{1}+q_{2}\right)\right) \\
\frac{\sin q_{2} \cos q_{2}}{\left(1+\sin ^{2} q_{2}\right)^{2}}\left[p_{1}^{2}-2 p_{1} p_{2}\left(1+\cos q_{2}\right)+p_{2}^{2}\left(3+2 \cos q_{2}\right)\right] \\
+\frac{1}{1+\sin ^{2} q_{2}}\left[-p_{1} p_{2} \sin q_{2}+p_{2}^{2} \sin q_{2}\right]-g \sin \left(q_{1}+q_{2}\right)
\end{array}\right) \\
& +\left(\begin{array}{cc}
0 & 0 \\
0 & 0 \\
1 & 0 \\
0 & 1
\end{array}\right)\left(\begin{array}{c}
u_{1} \\
u_{2}
\end{array}\right) \\
y_{1}= & q_{1}=h_{1} \\
y_{2}= & q_{2}=h_{2}
\end{aligned}
$$

Por outro lado temos que:

$$
\begin{aligned}
& \mathcal{L}_{f} h_{1}=\frac{-p_{1}-p_{2}\left(1+\cos q_{2}\right)}{1+\sin ^{2} q_{2}} \\
& \mathcal{L}_{f} h_{2}=\frac{-p_{1}\left(1+\cos q_{2}\right)+p_{2}\left(3+\cos q_{2}\right)}{1+\sin ^{2} q_{2}} \\
& \mathcal{L}_{g_{1}} h_{1}=\mathcal{L}_{g_{2}} h_{1}=\mathcal{L}_{g_{1}} h_{2}=\mathcal{L}_{g_{2}} h_{2}=0 .
\end{aligned}
$$

Estes são elementos do espaço de observação e a codistribuição de observabilidade está definida por:

$$
d \mathcal{O}(x)=\operatorname{Span}\{d h(x) \backslash h \in \mathcal{O} \text { onde } \quad x \in \mathcal{M}\}
$$


Portanto temos que:

$$
\begin{aligned}
d h_{1}= & d q_{1} \\
d h_{2}= & d q_{2} \\
d\left(\mathcal{L}_{f}\right) h_{1}= & \frac{1}{1+\sin ^{2} q_{2}}\left(\frac{2 \sin q_{2} \cos q_{2}}{1+\sin ^{2} q_{2}}\left(p_{1}+p_{2}\left(1+\cos q_{2}\right)\right)+p_{2} \sin q_{2}\right) d q_{2}-d p_{1} \\
& \left.+\left(1+\cos q_{2}\right) d p_{2}\right) \\
d\left(\mathcal{L}_{f} h_{2}\right)= & \frac{1}{1+\sin ^{2} q_{2}}\left(\frac{-2 \sin q_{2} \cos q_{2}}{1+\sin ^{2} q_{2}}\left(-p_{1}\left(1+\cos q_{2}\right)+p_{2}\left(3+\cos q_{2}\right)\right)\right. \\
& \left.\left.+\left(p_{1}-p_{2}\right) \sin q_{2}\right) d q_{2}-\left(1+\cos q_{2}\right) d p_{1}+\left(3+\cos q_{2}\right) d p_{2}\right)
\end{aligned}
$$

portanto podemos concluir que

$$
\operatorname{dimd} \mathcal{O}(q, p)=4 \quad \text { para todo }(q, p)
$$

que pelo Teorema (1.4.1) o sistema é localmente fracamente observável e pelo Corolário (3.5.1) é fortemente acessivel. 


\section{Referências Bibliográficas}

[1] Abraham, R and Marsden, J.E, "Foundation of Mechanics", Benjamin / Cummings, 1978.

[2] Arnold, V.I, "Mathematical methods of classical mechanics", New York, Springer, 1989.

[3] Brockett, R.W, "Control Theory and Analytical Mechanics" in Geometric Theory, edited by C. Martin and R. Hermann, Vol. VII of Lie Groups: History, Frontiers and Applications (Math. Sci. Press, Brookline, MA, 1977), pp 1-46.

[4] Brockett, R.W, "System theory on group manifold and coset spaces", SIAM J. Contr, Vol 10, pp 265-284, 1972.

[5] Elliot, D.L “ A consequence of controllability", J. Diff. Eqns, Vol 10, pp 364-370, 1970.

[6] Haynes, G.W and Hermes, H "Non-linear Controllability via Lie theory", SIAM J. Contr, Vol 8, pp 450-460, 1970.

[7] Hermann, R and Krener, A.J "Nonlinear controllability and observability", IEEE Trans. Aut. Contr, Vol AC-22, pp 728-740, 1970.

[8] Hermann, $\mathrm{R}$ " On the accessibility problem in control Theory", in Int. Symp. on Nonlinear Differential Equations and Nonlinear Mechanics, New York: Academic Press, pp 325-332, 1963.

[9] Isidori, A "Nonlinear control Systems" 3rd ed. Springer-Verlag London 1995.

[10] Kalman, R.E " On the general theory of control system", in Procedings of the First International Congress of the International Federation of Automatic Control, Vol 1, Butterworths, London,pp 481-492, 1960. 
[11] Kalman R.E "Contributions to the theory of optimal control", Sociedad Matematica Mexicana, Boletin. Segunda Serie, Vol 5 N 1, pp 102-119, 1960.

[12] Kreindler, E and Sarachick, P.E " On the concepts of controllability and observability of linear systems”, IEEE Trans. Aut. Contr, pp 129-136, April 1964.

[13] Krener, A.J " A generalization of Chows theorem and the bang-bang theorem to nonlinear control systems", SIAM J. Contr. Vol 12, pp 43-52, 1974.

[14] Libermann, P and Marle, C.M "Simpletic geometry and analytical mechanics, Dordrecht Reidel, 1987.

[15] Lobry, C "Controllabilité des systémes non linéaires", SIAM J. Contr, Vol \&, pp 573-605, 1970.

[16] Nijmeijer, H and Van der Schaft, A.J, "Nonlinear Dynamical Control Systems", Springer-Verlag, New York, 1990.

[17] Sontag, E.D, "Mathematical control theory: deterministic finite dimensional systems, New York, Springer, 1990.

[18] Sussmann, H.J and Jurdjevic, V.J "Controllability of nonlinear systems", J. Diff. Eqns, Vol 12, pp 95-116, 1972.

[19] Sussmann, H.J " Orbits of families of vector-fields and integrability of distributions", Trans. American Math. Soc., Vol 180, pp 171-188, 1973.

[20] Sussmann, H.J "Existence and uniqueness of minimal realizations of nonlinear systems", Math. Syst. Theory 10, pp 263-284, 1977.

[21] Sussmann, H.J "A generalization of the closed subgroup theorem to quotients of arbitrary manifold”, J. Diff. Geometry, Vol 10, pp 151-166, 1975.

[22] Sussmann, H.J "Lie brackets, real analyticity and geometric control", in Differentian Geometric control Theory (Eds. R.W Brockett, R.S Millman, H.J Sussmann), pp 1-116. Birkhäuser, Boston, 1983.

[23] Van der Schaft, A.J "Observability and Controllability for smooth nonlinear systems", SIAM J Control and Optimization, Vol $20 N^{\mathrm{O}}$ 3, pp 338-354, 1982.

[24] Van der Schaft, A.J "Hamiltonian dynamics with external forces and observations", Math. Syst. Theory, Vol 15, pp 145-168, 1982. 
[25] Van der Schaft, A.J "Controllability and observability for affine nonlinear Hamiltonian systems", IEEE Trns. Automat. Contr., Vol AC-27 Noㅡ 2, pp 490-492, 1982.

[26] Willems, J.C "System theoretic models for the analysis of physical systems", Ricerche di Automatica, Special Issue on Systems Theory and Physich, Vol 10, pp 71-106, Dec. 1979. 